Florida International University FIU Digital Commons

7-29-2017

\title{
Multi-resolution Modeling of Dynamic Signal Control on Urban Streets
}

Aidin Massahi

4246488, amass025@fiu.edu

DOI: $10.25148 /$ etd.FIDC001982

Follow this and additional works at: https://digitalcommons.fiu.edu/etd

Part of the Transportation Engineering Commons

\section{Recommended Citation}

Massahi, Aidin, "Multi-resolution Modeling of Dynamic Signal Control on Urban Streets" (2017). FIU Electronic Theses and Dissertations. 3349.

https://digitalcommons.fiu.edu/etd/3349

This work is brought to you for free and open access by the University Graduate School at FIU Digital Commons. It has been accepted for inclusion in FIU Electronic Theses and Dissertations by an authorized administrator of FIU Digital Commons. For more information, please contact dcc@fiu.edu. 
FLORIDA INTERNATIONAL UNIVERSITY

Miami, Florida

\title{
MULTI-RESOLUTION MODELING OF DYNAMIC SIGNAL CONTOROL ON URBAN STREETS
}

\author{
A dissertation submitted in partial fulfillment of \\ the requirments for the degree of \\ DOCTOR OF PHILOSOPHY
}

in

CIVIL ENGINEERING

by

Aidin Massahi 
To: Interim Dean Ranu Jung

College of Engineering and Computing

This dissertation, written by Aidin Massahi, and entitled Multi-Resolution Modeling of Dynamic Signal Control on Urban Streets, having been approved in respect to style and intellectual content, is referred to you for judgment.

We have read this dissertation and recommend that it be approved.

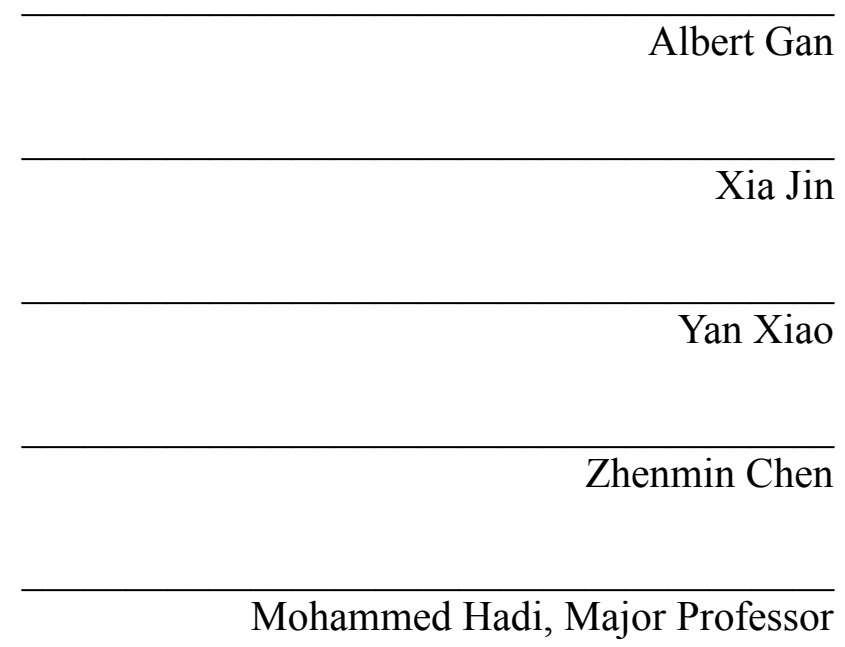

Date of Defense: June 29, 2017

The dissertation of Aidin Massahi is approved.

Interim Dean Ranu Jung

College of Engineering and Computing

Andrés G. Gil

Vice President for Research and Economic Development and Dean of the University Graduate School

Florida International University, 2017 
(C) Copyright 2017 by Aidin Massahi All Rights Reserved. 


\section{DEDICATION}

I dedicate this dissertation to my beloved parents, Jaleh and Abdolazim, and my lovely brothers Arman and Arash for their unconditional love and their endless support and encouragement. 


\section{ACKNOWLEDGMENTS}

First and foremost, I would like to thank my advisor, Dr. Mohammed Hadi, for his guidance, patience. It was my honor to work with such a well-respected researcher in the Intelligent Transportation Systems (ITS) field. He was generous with the time he spent on advising me and training me to conduct high quality research, and to develop a variety of skills that are necessary to succeed in my professional career.

I experienced a great teamwork environment in the Integrated Intelligent Transportation Systems (IITS) research lab. I would like to thank Dr. Yan Xiao, who is incredibly wise, knowledgeable, and kind to share her knowledge. I appreciate her continued support of me in different endeavors, including leading student chapters at Florida International University. I would like extend my deep appreciation to Dr. Hesham Ali, who I learned much from in different pavement engineering topics. In addition, I would like to thank Dr. Gan for teaching me how much I need to be in detail and how to be a perfect writer. However, I do not gratefully thank him for killing me to sign my ETD form.

I would also like to thank my friend and colleague, Dr. Kollol Shams, Homa Fartash, Shahadat Iqbal, Samaneh Khazraian, Dr. Tao Wang, Dr. Mohammad Lavasani, Dr. Hamid Asghari and Eazaz Sadeghvaziri for their valuable advice on professional and academic development. I will be always thankful to my dear friends Elmira, Kaveh, Peyman, Saman, Mojtaba, Mohmmadtaghi, Pedram, for their assistance, friendship, care and encouragement.

Finally, I would also like to thank the U.S. and Iran diplomatic relationship especially under Trump administration that rejected my parents visa and putting me in big 
prison as wide as US. I am really homesick and burnt out because of this political atmosphere. 


\section{ABSTRACT OF THE DISSERTATION \\ MULTI-RESOLUTION MODELING OF DYNAMIC SIGNAL CONTROL ON URBAN STREETS}

by

Aidin Massahi

Florida International University, 2017

Miami, Florida

Professor Mohammed Hadi, Major Professor

Dynamic signal control provides significant benefits in terms of travel time, travel time reliability, and other performance measures of transportation systems. The goal of this research is to develop and evaluate a methodology to support the planning for operations of dynamic signal control utilizing a multi-resolution analysis approach. The multiresolution analysis modeling combines analysis, modeling, and simulation (AMS) tools to support the assessment of the impacts of dynamic traffic signal control.

Dynamic signal control strategies are effective in relieving congestions during nontypical days, such as those with high demands, incidents with different attributes, and adverse weather conditions. This research recognizes the need to model the impacts of dynamic signal controls for different days representing, different demand and incident levels. Methods are identified to calibrate the utilized tools for the patterns during different days based on demands and incident conditions utilizing combinations of real-world data with different levels of details. A significant challenge addressed in this study is to ensure that the mesoscopic simulation-based dynamic traffic assignment (DTA) models produces 
turning movement volumes at signalized intersections with sufficient accuracy for the purpose of the analysis. Although, an important aspect when modeling incident responsive signal control is to determine the capacity impacts of incidents considering the interaction between the drop in capacity below demands at the midblock urban street segment location and the upstream and downstream signalized intersection operations. A new model is developed to estimate the drop in capacity at the incident location by considering the downstream signal control queue spillback effects. A second model is developed to estimate the reduction in the upstream intersection capacity due to the drop in capacity at the midblock incident location as estimated by the first model. These developed models are used as part of a mesoscopic simulation-based DTA modeling to set the capacity during incident conditions, when such modeling is used to estimate the diversion during incidents. To supplement the DTA-based analysis, regression models are developed to estimate the diversion rate due to urban street incidents based on real-world data. These regression models are combined with the DTA model to estimate the volume at the incident location and alternative routes. The volumes with different demands and incident levels, resulting from DTA modeling are imported to a microscopic simulation model for more detailed analysis of dynamic signal control. The microscopic model shows that the implementation of special signal plans during incidents and different demand levels can improve mobility measures. 


\section{TABLE OF CONTENTS}

CHAPTER

PAGE

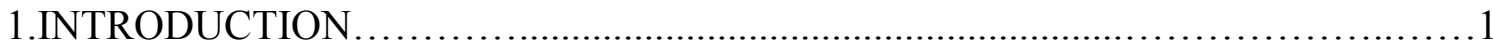

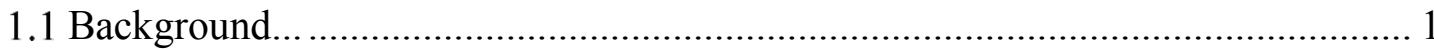

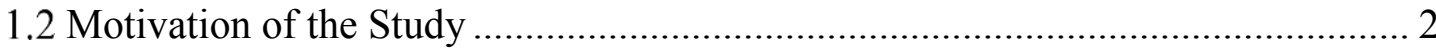

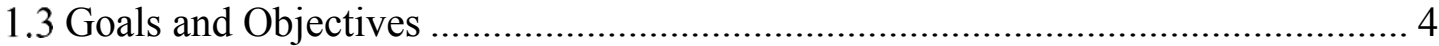

1.4 Dissertation Organization ............................................................................... 4

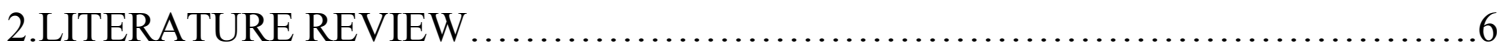

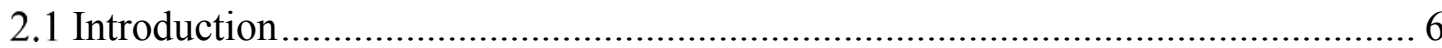

2.2 Planning and Evaluating Active Traffic And Demand Management Strategies .... 6

2.2.1 Active Transportation Management Strategies ................................... 8

2.3 Multi-Resolution Analyses Tools and Methods .................................................11

2.3.1 Sketch Planning/Spreadsheet Methods ............................................. 12

2.3.2 Macroscopic Models ..................................................................... 14

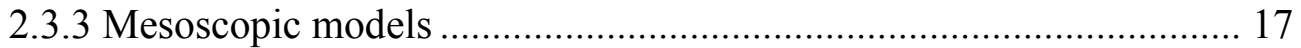

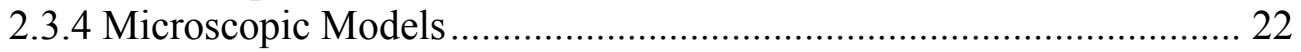

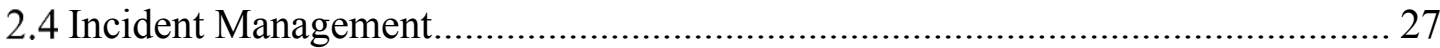

2.4.1 Impact of Incident Management on Incident Duration ....................... 28

2.4.2 Impact of Incident Management on Diversion Rate ........................... 32

2.4.3 Impact of Incident Management on Safety ………………………...... 33

2.4.4 Incident and Incident Management Modeling in Existing Tools ......... 33

2.5 Signal Control Strategies and Their Effects on Network ……………………..... 36

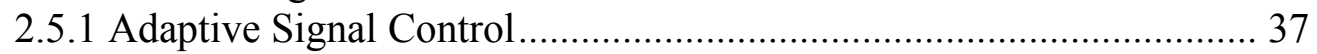

2.5.2 Time of Day Signal Control Retiming .............................................. 38

2.5.3 Comparison Adaptive Signal Timing with Retiming Signal Control .. 41

2.5.4 Impact of Signal Timing Strategies on the Network Performance

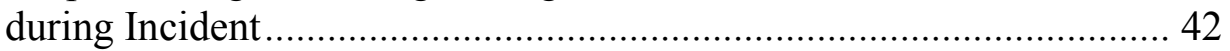

2.5.5 Weather-Response Signal Control .................................................... 44

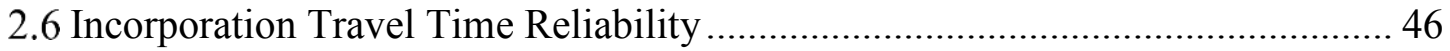

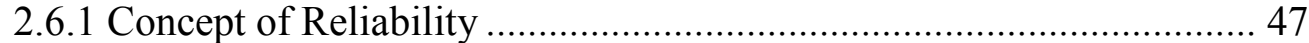

2.6.2 Source of Travel Time Unreliability ................................................ 49

2.6.3 Incorporating Reliability into Operations Modeling Tools .................. 49

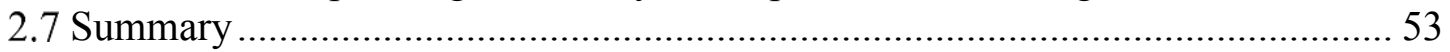

3. METHODOLOGY AND OVERVIEW OF MODELING PROCESS....................55

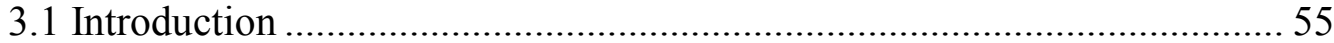

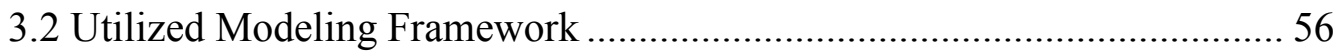

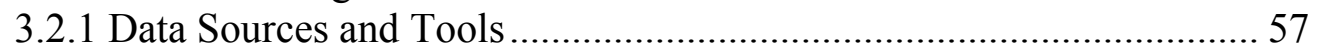

3.2.2 Modeling Support Environment...................................................... 59 
3.3 Network Preparation and Calibration for Multiresolution Analyses.......... 59

3.4 Traffic and Incident Responsive Signal Control Modeling......................... 61

4.DTA MODEL PREPARATION AND CALIBRATION............................. 63

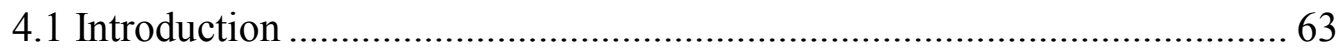

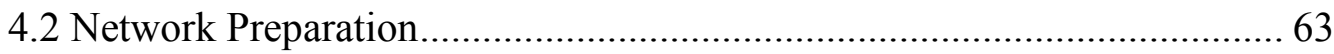

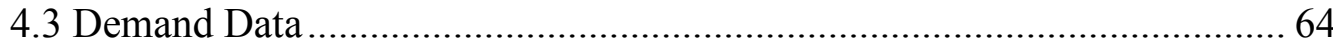

4.4 Network and Demand Data Conversion to DTALite ...................................... 65

4.5 Construction of signal and Junction Input Data ............................................. 66

4.6 Data Acquisition and Traffic Pattern Identification ...................................... 68

4.7 Supply Calibration................................................................................ 71

4.8 Demand Calibration .............................................................................. 73

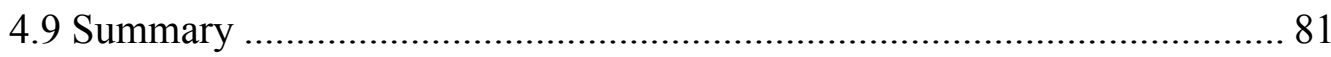

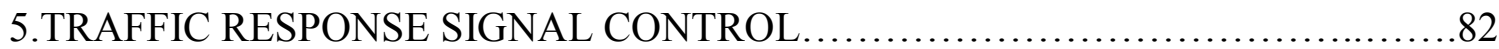

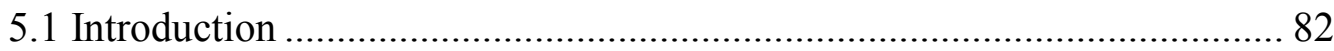

5.2 Calibration of Turning Movement Volumes ............................................... 83

5.3 HCS Signal Optimization on Network Performance .................................. 87

5.4 Network Modeling in Microscopic Simulation........................................... 88

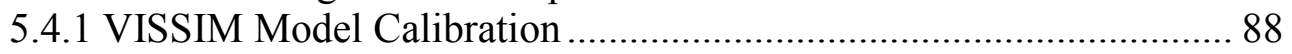

5.4.2 Volume Representation in VISSIM .................................................. 91

5.5 Microscopic Simulation Assessment of Demand Responsive Signal..... 95

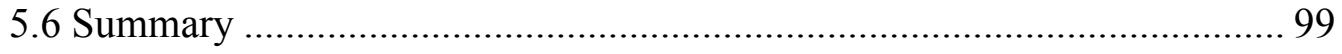

6. INCIDENT RESPONSE SIGNAL CONTROL .................................101

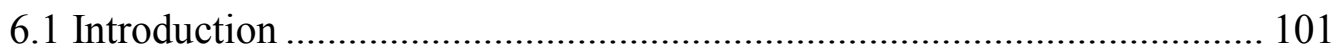

6.2 Estimating the Capacity Impacts of Urban Street Incidents...................... 102

6.2.1 Procedure of Estimating Incident Capacity in Urban Street ............ 105

6.2.2 Calibration of Microscopic Simulation for No-Incident Conditions 105

6.2.3 Estimating Capacity Drop due to Incidents at the Movement Stop

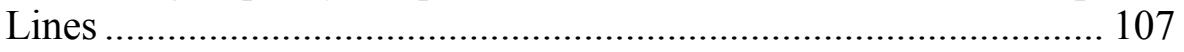

6.2.4 Modeling Incidents in Microscopic Simulation .............................. 109

6.2.5 Assessing the Impacts of Downstream signal on Upstream Incident

Capacity ..................................................................................... 109

6.2.6 Assessing the Impacts of Incidents on Upstream Intersection Maximum Throughputs .............................................................. 112

6.3 Estimation of Incident Diversion Rate ................................................... 118

6.3.1 Traffic Volume Estimation for No Incident Days ........................... 120

6.3.2 Diversion Rate Estimation......................................................... 122

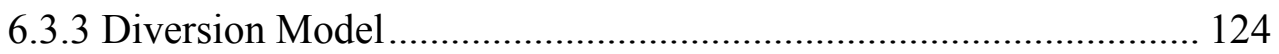

6.4 Modeling Incidents in Mesoscopic Simulation ......................................... 125

6.5 Modeling of Diversion in the DTA Tool................................................. 129 
6.6 Microscopic Simulation of Special Signal Plans during Incidents .......... 131

6.6.1 Special Signal Timing Plan .................................................. 131

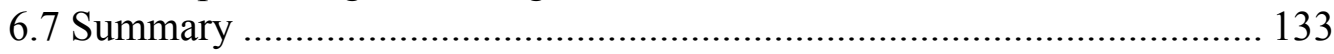

7. ESTIMATING INCIDENT IMPACTS ON URBAN STREET TRAVEL TIME....135

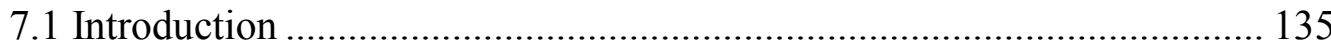

7.2 Estimating Incident Impact on Delay ............................................... 136

7.3 Capacity of Urban Street Segments..................................................... 138

7.4 Arterial Delay Estimation Accuracy Assessment Results...................... 140

7.5 Model Utilization for Decision Support ............................................. 142

7.6 Model Utilization for Decision Support .............................................. 145

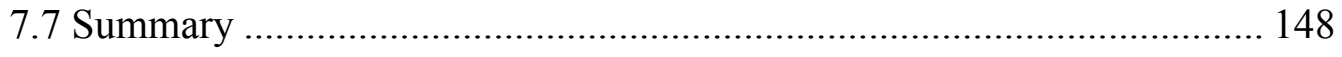

8. CONCLUSION AND RECOMMENDATIONS FOR FUTURE STUDIES ..........150

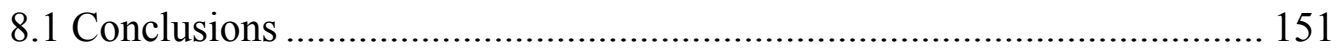

8.2 Recommendations for Future Works ............................................... 154

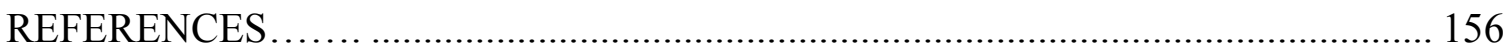

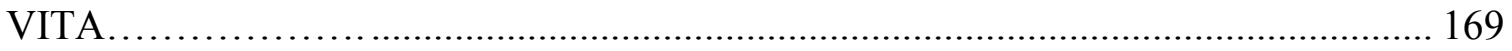




\section{LIST OF TABLES}

TABLE

PAGE

Table 2-1 Examples of ATDM Strategies......................................

Table 2-2 Definitions and Use of Reliability Performance Measures ....................... 49

Table 4-1 Simulated Turning Movement Counts vs. Observed Turning Movement Counts for Broward Boulevard for Different Levels of Demand ............ 80

Table 4-2 Simulated Turning Movement Counts vs. Observed Turning Movement Counts for Broward Boulevard for Three Observed Volume Classes ......... 81

Table 5-1 Default and Calibrated Parameters............................................ 91

Table 5-2 The Performance of Optimizing Control Plan for the 75th Percentile of Demand Level vs. Using the Optimized Plan for the Median Day............. 97

Table 5-3 The Performance of Optimizing Control Plan for the 95th Demand Levelvs. Using the Optimized Plan for the Median Day................. 97

Table 5-4 The Benefits of the More Dynamic Responsive Control for the 75th Percentile of Demand................................................ 98

Table 5-5 The Benefits of the More Dynamic Responsive Control for the 95th Percentile of Demand............................................. 99

Table 6-1 Upstream Interrupted Incident Capacity Regression Models..................... 112

Table 6-2 Variation in Upstream Unconstrained Green in Terms of Incident location, Duration and Volume to Incident Capacity (v/Ic) Ratio..

Table 6-3 Regression Models to Estimate Upstream Intersection Maximum Throughput and Unconstrained Green Regression Models............... 118

Table 6-4 Upstream Signal Maximum Throughput for the Test Scenarios............... 129

Table 6-5 Traffic Response Special signal Timing vs. Normal Signal Timing.......... 133

Table 7-1 Regression Models to Estimate the Interaction Between Drop in Capacity due to Incidents and Upstream and Downstream Signal Operations 
Table 7-2 Network Delay Saving (Vehicle-hrs) due to Upstream Signal Timing Plan Adjustment During the Incident Condition in VISSIM and the Proposed Analytical Model.......................................... 148 


\section{LIST OF FIGURES}

FIGURE

Figure 2-1 Monte Carlo Approach.............................................. 51

Figure 2-2 Mix and Match Approach............................................... 52

Figure 3-1 Utilized Modeling Framework.......................................... 57

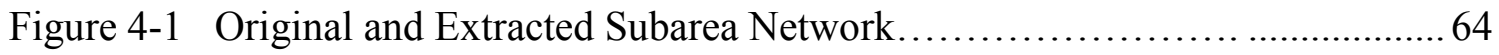

Figure4-2 GIS importing setting Interface in NeXTA................................ 66

Figure 4-3 Example of Link Adjustment in NeXTA...................................... 67

Figure4-4 "Node Movement Data" Interface for the Intersection at the Broward Boulevard and US-1 in NeXTA........................................ 68

Figure 4-5 Average Volume During the PM Peak Period for Normal Days from June

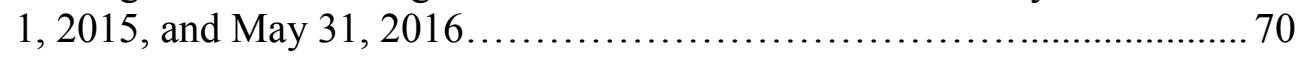

Figure 4-6 Variation of Average Volume for Days with Different Percentiles........... 72

Figure 4-7 Simulated Link vs. Observed Link Volumes Before and After Running ODME for Day with $50^{\text {th }}$ Percentile of Demand........................ 74

Figure 4-8 Simulated Link vs. Observed Link Volumes Before and After Running ODME for Day with $75^{\text {th }}$ Percentile of Demand............................ 74

Figure 4-9 Simulated Link vs. Observed Link Volumes Before and After Running ODME for Day with $95^{\text {th }}$ Percentile of Demand......................... 75

Figure 4-10 Virtual Sensor Locations in the Study Network............................. 77

Figure 4-11 Simulated vs. Observed Link Volumes, Before and After Running ODME for the Day with $50^{\text {th }}$ Percentile of Demand.............................. 78

Figure 4-12 Simulated Link vs. Observed Link Volumes, Before and After Running ODME for the Day with $75^{\text {th }}$ Percentile of Demand........................... 78

Figure 4-13 Simulated Link vs. Observed Link Volumes, Before and After Running ODME for the Day with $95^{\text {th }}$ Percentile of Demand......................... 79 
Figure 5-1 Turning Movement Sensor Adjustment in NeXTA............................... 85

Figure 5-2 MAE (Upper Numbers) and MAPE (Lower Numbers) for Each Left, Through and Right Turning Movements for Day with $50^{\text {th }}$ Percentile of

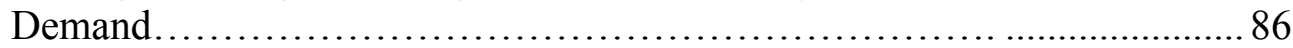

Figure 5-3 MAE (Upper Numbers) and MAPE (Lower Numbers) for Each Left, Through and Right Tirning Movements for Day with $75^{\text {th }}$ Percentile of Demand

Figure 5-4 MAE (Upper Numbers) and MAPE (Lower Numbers) for Each Left, Through and Right Turning Movements for Day with $95^{\text {th }}$ Percentile of Demand

Figure 5-5 Relationship Between Saturation Flow Rate and Desired Safety Distance Parameters........................................................ 90

Figure 5-6 Case Study Network....................................................... 92

Figure 5-7 Link Based Assignment vs. Path Based Assignment............................... 93

Figure 5-8 Comparison Between Path Based Assignment and Link Based Assignment for the Median (50\%) Demand Day.

Figure 5-9 Comparison of Delay and Travel Time Between Path Based Assignment and Link Based Assignment............................................... 95

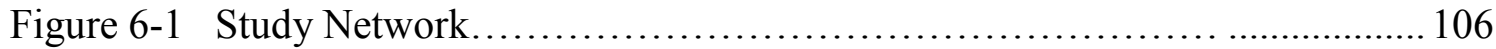

Figure 6-2 Interrupted Incident Capacity Effected by Downstream Intersection......... 111

Figure 6-3 Upstream Intersection Maximum Throughput Variation with Incident Location And Duration $(\mathrm{v} / \mathrm{Ic}=1.13)$ at the Incident Location.............. 114

Figure 6-4 Upstream Intersection Maximum Throughput Variation with Incident Location and Duration $(\mathrm{v} / \mathrm{Ic}=1.3)$ at the Incident Location................. 114

Figure 6-5 Upstream Intersection Maximum Throughput Variation with Incident Location and Duration $(\mathrm{v} / \mathrm{Ic}=1.5)$ at the Incident Location.

Figure 6-6 Broward Blvd Eastbound Volume Distribution (PM Peak)

Figure 6-7 Sunrise Blvd Eastbound Volume Distribution 
Figure 6-8 Two Examples Of Broward \& Sunrise Normal and Incident Day Counts

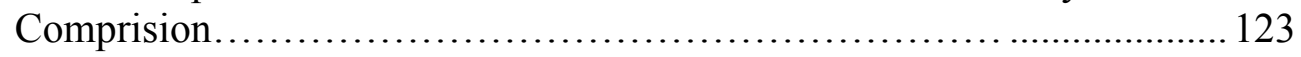

Figure 6-9 Relationship Between Average Diversion Rate and Incident Duration..... 125

Figure 6-10 Drop the Incident Upstream Links' Capacities.................................. 131

Figure 6-11 Signal Control Plan for Normal Day vs. Special Signal Control Plan....... 132

Figure 7-1 Comparison of Incident Delay Using the Proposed Method, Queuing Analysis, and VISSIM............................................. 142 
ABBREVIATIONS AND ACRONYMS

\begin{tabular}{ll} 
ADM & Active Demand Management \\
APM & Active Parking Management \\
ATCS & Adaptive Traffic Control System \\
ATM & Active Traffic Management \\
ATDM & Active Transportation and Demand Management \\
CHART & Coordinated Highways Action Response Team \\
DMS & Dynamic Message Signs \\
DTA & Dynamic Traffic Assignment \\
FETSIM & Fuel Efficient Traffic Signal Management \\
FHWA & Federal Highway Administration \\
FSP & Freeway Service Patrol \\
HAR & Highway Advisory Radio \\
HOT & High Occupancy Tolling \\
IDAS & ITS Deployment Analysis System \\
MSA & Method of Successive Averages \\
O-D & Origin-Destination \\
ODME & Origin-Destination Matrix Estimation \\
PTMS & Portable Traffic Monitoring Sites \\
SERPM & Southeast Florida Regional Planning Model \\
\hline
\end{tabular}


SPC Southwestern Pennsylvania Commissions

VSL Variable Speed Limits

VOR Value of Travel Time Reliability

VOT Value of Travel Time 


\section{CHAPTER 1 \\ INTRODUCTION}

\subsection{Background}

Active Traffic Management (ATM) is an important component of Transportation System Management and Operations (TSM\&O) and Active Traffic Demand Management (ATDM), providing significant benefits in terms of travel time, travel time reliability, emission, fuel consumption, safety, and other performance measures of the transportation systems. There is an urgent need for decision support systems to support ATM strategies. This is particularly true for ATM strategies on urban streets, as agencies start moving their focus to these streets. Agencies have encountered other challenges, including their need to identify the urban street corridors that benefit the most from ATM implementation, what strategies are the most advantageous, in addition to the magnitude of the benefits, costs and their implementation barriers under different conditions and unreliability sources such as incidents, high demands and severe weathers conditions.

Agencies are also interested in assessing the benefits and costs in investing in ATM strategies versus investing in capacity improvements such as adding lanes or interchange modifications. Therefore, ATM strategies are becoming crucial components of transportation system planning and operations. Various levels of modeling tools including sketch planning, macroscopic, mesoscopic, microscopic simulations tools combined with static or dynamic traffic assignment have been applied by transportation agencies to model advanced traffic management strategies. 
Multi-resolution modeling (MRM) is an integrated approach that combines different modeling levels that can be used as an effective method for the assessment and support of ATM strategies and to support mitigating transportation system congestion problems. However, there is a need for methods and tools to allow agencies to use MRM in general and to use MRM in evaluating ATM in particular. This dissertation develops and uses methods for the use of MRM to support agency decisions related to ATM strategies deployment on urban streets.

\subsection{Motivation of the Study}

In recent years, the implementations of ATM have increased dramatically. As a result, there has been an increasing interest in assessing the benefits of ATM strategies on urban streets. Methods are needed to assess the impacts of ATM strategies particularly on urban streets. The impacts of ATM strategies depend on the network characteristics including demand levels and available capacity, signal configuration and timing, and event types and frequencies.

MRM can be used as a powerful approach, combining different modeling levels and associated methods to model ATM strategies and estimate their performances. One of the important elements of MRM is simulation-based Dynamic Traffic Assignment (DTA). DTA is capable of realistic modeling of traffic flow and driver responses to network conditions and ATM strategies. One of the main advantages of using DTA to model traffic networks is its ability to model the time-dependent network states. Outputs from DTA models include time-dependent system level, route level, and link level performance 
statistics. In addition, vehicle trajectories output by these models can be processed to produce even more detailed statistics than those output by the conventional models. From these statistics, a set of metrics can be derived in order to study system performance and provide inputs to other higher resolution models, if necessary.

Simulation-based DTA models can be used to model ATM strategies. However, additional modeling capabilities in a MRM platform are needed to allow a detailed assessment of these strategies. Micro-simulation is needed to model in more details specific arterial facilities. A signal optimization model is needed to optimize signal control and help to emulate signal control strategies. Models and methods will also be needed to support the estimation of performance measures such as reliability, mobility, safety and environmental measures based on model outputs. There has been an increasing interest in recent years of the need for the use of combinations of modeling tools in an integrated manner to model ATM strategies.

In general, the deployment of ATM can improve traffic flow and decrease congestion, thereby improving system performance. The evaluation of ATM strategies has been done for one day that is assumed to be representative of the operations for the whole year. The ATM evaluation requires multi-scenario modeling that involves the assessment of the impacts of variations in demand, weather, congestion, incident, and other events on system performance. The multi-scenario analysis requires analyzing scenarios with a wide range of variations in the factors that influence performance. 


\subsection{Goals and Objectives}

The goal of this project is to develop methods to support the use of MRM in the assessment of ATM strategies, with specific emphasis on dynamic traffic signal control. The specific objectives are as follows:

Objective 1: Develop methods that utilize combinations of advanced simulation, DTA, and optimization models to allow the effective assessment of dynamic signal control, including demand responsive and incident responsive signal control

Objective 2: Develop models to estimate arterial system performance under incident conditions with consideration of the interaction between the operations at the incident location and upstream and downstream signalized intersection operations to support of the assessment of ATM strategies under incident conditions

Objective 3: Develop methods to estimate the diversion behavior of drivers under incident conditions in support of the assessment of ATM strategies under incident conditions

Objective 4: Investigate methods to better estimate volumes from DTA tools for use as inputs to signal optimization and microscopic simulation models.

\subsection{Dissertation Organization}

The remainder of this dissertation is structured as follows: Chapter II presents a review and assessment of past research related to the objectives and tasks of this study. Chapter III presents the methodology and procedures that have been carried out to support 
achieving the objectives of this study. Chapter IV discusses the network preparation and calibration of the mesoscopic simulation-based DTA model utilized in this study.

Chapter $\mathrm{V}$ describes the modeling of demand responsive control. A combination of a mesoscopic simulation-based DTA tool (DTALite), microscopic simulation (VISSIM), and a signal timing optimization tool was used to derive and evaluate demand responsive signal plans. The methodology, procedure, and results from this modeling discussed in this Chapter.

Chapter VI describes a new approach and models to estimate the capacity of the interaction between the drop in capacity below demands at midblock urban streets segment locations and upstream and downstream signalized intersection operations. These models were implemented to refine the DTA modeling during incident conditions. This chapter also discusses the development of regression models to estimate diversion due to urban street incidents. These regression models were combined with the DTA model to estimate the volumes at the incident location and alternative routes. The produced volumes during the incident were then used as inputs to microscopic simulation for more detailed analysis, as well as to demonstrate the benefits of special signal plans during incident conditions.

Chapter VII presents the method used to estimate delay and travel time due to incident impacts on the urban streets. The chapter then discusses the incorporation of the method in a data analytic tool and its use to inform the identification of a special signal timing plan to reduce incident impacts. Chapter VIII summarizes the findings of this research. 


\section{CHAPTER 2}

\section{LITERATURE REVIEW}

\subsection{Introduction}

This chapter presents a review and assessment of all of the relevant topics to the modeling of advanced strategies. The review is grouped into five related parts to the objective and tasks of this study. First, a review is presented of Active Traffic Demand Management (ATDM) strategies on urban streets. The second part provides information about the Multi-resolution framework, modeling and available tools. The third part is an overview of the impacts of incidents and incident management strategies on urban streets. The fourth part is a review of the benefits of adaptive control, time of day signal retiming and incident response, and weather responsive signal plans to relieve the traffic congestion. Next, a discussion is presented of the modeling framework of travel time reliability, including mathematical formulations, theories and analytical techniques.

\subsection{Planning and Evaluating Active Traffic And Demand Management Strategies}

ATDM strategies dynamically control and manage traffic demand, travel demand, and traffic flow of transportation facilities (Sheehban et al., 2012; Rouphail et al., 2016; Aghdashi et al., 2017; Khazraeian et al., 2015; Khazraeian et al., 2017). The Federal Highway Administration's (FHWA) ATDM programs was introduced to support active, integrated and performance-based solutions to enhance safety, maximize system productivity, and improve individual mobility in multi-modal surface transportation 
systems. Examples of ATDM strategies are shown in Table 2-1 (Mohammed Hadi et al., 2016).

Table 2-1 Examples of ATDM Strategies

\begin{tabular}{|c|c|c|}
\hline $\begin{array}{c}\text { Active Traffic } \\
\text { Management (ATM) }\end{array}$ & $\begin{array}{c}\text { Active Demand } \\
\text { Management (ADM) }\end{array}$ & $\begin{array}{c}\text { Active Parking } \\
\text { Management (APM) }\end{array}$ \\
\hline Adaptive Ramp Metering & Dynamic Fare reduction & $\begin{array}{c}\text { Dynamic Overflow } \\
\text { Transit Parking }\end{array}$ \\
\hline $\begin{array}{c}\text { Adaptive Traffic Signal } \\
\text { Control }\end{array}$ & $\begin{array}{c}\text { Dynamic HOV/Managed } \\
\text { Lanes }\end{array}$ & $\begin{array}{c}\text { Dynamic Parking } \\
\text { Reservation }\end{array}$ \\
\hline Dynamic Junction Control & Dynamic Pricing & Dynamic Wayfinding \\
\hline $\begin{array}{c}\text { Dynamic Lane Reversal or } \\
\text { Contraflow Lane Reversal }\end{array}$ & $\begin{array}{c}\text { Dynamic Ridesharing } \\
\text { Dynamic Lane Use Control }\end{array}$ & Dynamic Priced Parking \\
\hline Dynamic Merger Control & $\begin{array}{c}\text { Dynamic Transit Capacity } \\
\text { Assignment }\end{array}$ & \\
\hline Dynamic Shoulder Lanes & On-Demand Transit & \\
\hline Dynamic Speed Limits & $\begin{array}{c}\text { Predictive Traveler } \\
\text { Information }\end{array}$ & \\
\hline Queue Warning & $\begin{array}{c}\text { Transfer Connection } \\
\text { Protection }\end{array}$ & \\
\hline Transit Signal Priority & & \\
\hline
\end{tabular}

In recent years, the deployment of ATDM has increased dramatically. As a result, there has been an increasing interest in assessing the benefits of ATDM strategies on urban streets. This is because most regions in the United States have already deployed ITS devices and associated strategies on freeways. As they start moving to deployments on signalized arterials, these strategies encounter other challenges, including the need to identify urban street corridors that benefit the most from the implementations, what strategies are the most advantageous under different conditions, and the magnitude of the benefits and costs under different situations. The reaming section will review ATDM strategies with a particular focus on urban strategies. It should be noted that the focus of 
this study is on ATM, which does not include the active demand management (ADM) and active parking management (APM) of ATDM.

\subsubsection{Active Transportation Management Strategies}

ATM strategies on urban streets maximize the productivity and efficiency of a signalized facility while increasing throughput and improving mobility, safety, reliability and sustainability. ATM strategies are systems that integrate technologies and strategies to optimize system performance.

\subsubsection{Adaptive Ramp Metering}

Adaptive ramp metering dynamically controls the rate at which vehicles enter a freeway facility. It can be used in order to respond to recurrent and non-recurrent events. Although this strategy by itself is considered a freeway ATM strategy, it can be integrated with adjacent arterial traffic signals. Previous studies show that adaptive ramp metering has improved both safety and mobility, including drops of $43 \%, 39 \%, 24 \%$ and $15 \%$ in collision rates in Portland, Seattle, Minneapolis, and Long Island during peak hours. The average travel times and travel speeds have also improved in these areas (Sorrell 2014).

\subsubsection{Adaptive Traffic Signal Control}

Adaptive signal control technology dynamically adjusts the signal timing to accommodate changing traffic patterns in order to minimize overall delays (Lardoux et al., 2014; Sadeghvaziri et al., 2016; Rojas et al., 2016). Adaptive traffic signal control usually collects upstream traffic flow parameters, and then continuously modifies timing 
parameters. Adaptive signal control has resulted in a 10 to $41 \%$ reduction in stops at traffic signals, and a 5 to $42 \%$ reduction in delays (FHWA 2016).

\subsubsection{Dynamic Junction Control}

Dynamic junction control dynamically assigns the lane assignments on the mainline and ramps in the vicinity of merge and diverge areas. The volumes on the mainline lanes and ramps are always monitored. Based on real-time and anticipated conditions, the lane assignment is dynamically changed. This strategy can be considered a freeway ATDM.

\subsubsection{Dynamic Lane Reversal or Contraflow Lane Reversal}

Dynamic contra-flow, or lane reversal, involves the reversal of traffic flow in a lane or a shoulder in one direction for use by traffic in the other direction. Studies show an increase in capacity between 8 to $70 \%$, depending on the reserved type and original number of lanes (Sisiopiku et al., 2010). This strategy has mainly been implemented for freeways. However, it is also applicable for arterial strategies.

\subsubsection{Transit Signal Priority}

The management of traffic signals detects a bus near a signal control and then extends the green phase or changes the signals to green sooner, thus allowing the bus to pass the intersection more quickly. Previous studies show a $40 \%$ reduction in bus delays in Tacoma, Washington; a $10 \%$ and $19 \%$ improvement in travel time and travel time reliability of buses in Portland, Oregon, and Los Angeles and Chicago implementations show a $25 \%$ and $15 \%$ reduction in transit travel times, respectively (Sorrell 2014). 


\subsubsection{Variable Speed Limits (VSL)}

Variable speed limit (VSL) strategies dynamically adjust speed limits based on realtime traffic, roadway and weather conditions. From a safety point of view, they were shown to reduce crash rates between $10 \%$ and $50 \%$. Studies show a $2 \%$ to $10 \%$ reduction in emissions because of VSL. VSL can help stabilize traffic flow and slightly increase capacity (up to 5\%) (Sorrell 2014; Abdi et al., 2014).Variable speed limits have mainly been used for freeways.

\subsubsection{Dynamic Shoulder Lanes}

The use of shoulders as travel lanes during peak periods or during incidents or other events is known as hard shoulder running or temporary shoulder use (Lardoux et al., 2014). This is mainly a limited access facility strategy. Previous studies show a $20 \%$ to $30 \%$ increase in capacity in Germany; a 7\% to $16 \%$ improvement in capacity in France; and a $7 \%$ to $22 \%$ increase in capacity in the Netherlands. Washington State hard shoulder implementations show that average speeds increased from $10 \mathrm{mph}$ to $37 \mathrm{mph}$ (M.Rouphail, et al., 2015).

\subsubsection{Dynamic Merge Control}

Dynamic late merge or dynamic early merge consists of a series of strategies that dynamically manage the entry of vehicles into merge zones with a series of advisory messages. Dynamic merge control can provide safe merging gaps and decrease shockwaves upstream of merge points. The results of a pilot study on interchange merge 
control in the Netherlands shows that the mean travel time and vehicle hours of delay decreased around 8 and $13 \%$ respectively, and the mean travel speed increased $8 \%$ due to this strategy (Hellernan 2010). This strategy has been mainly used for freeways, although it can also be used for arterial strategy.

\subsubsection{Dynamic Lane Use Control}

Dynamic lane use control strategies provide advanced warning of closure(s), usually by dynamic lane control signs in order to safely merge traffic into adjacent lanes. This strategy dynamically manages the location to reduce rear-end and other secondary crashes (Lardoux et al., 2014). A special application of dynamic lane control that is particularly applicable to arterial streets is to dynamically assign the approach lanes between the turning movements by time of day or based on traffic demands.

\subsubsection{Queue Warning}

Queue warning signs are installed along a roadway to alert motorists of the presence of queues ahead in order to reduce rear-end crashes and improve safety. An analysis of rear-end crashes was conducted in Madison County, Illinois. The results show a $13.8 \%$ reduction in this type of crash, even though there was a $25.4 \%$ increase in traffic exposure in the post-deployment conditions (Roelofs and Brookes 2014).

\subsection{Multi-Resolution Analyses Tools and Methods}

Modeling of transportation systems is becoming a vital part of the planning and operations of these systems. Transportation modeling tools are commonly categorized as 
sketch planning, macroscopic simulation, mesoscopic simulation, and microscopic simulation tools based on the resolution at which vehicle flows are simulated. Many of these models have also DTA components. This section provides an overview of some of the available sketch planning, simulation-based tools and DTA tools with different levels of associated traffic models.

\subsubsection{Sketch Planning/Spreadsheet Methods}

There are a number of available sketch planning tools that can be used for assessing ATM strategies. These tools provide benefit and cost analyses for assessing ITS alternatives at high levels. The results are less accurate than using more advanced approaches, such as utilizing simulation and DTA. These tools have varying degrees of complexity. This section provides a brief review of two existing ITS evaluation tools. The Florida ITS Evaluation tool (FITSEVAL), and the TOPS-BC tool are examples of two different levels of tool complexity. There are several other similar tools available that are not reviewed in this document.

\subsubsection{Florida ITS Evaluation Tool (FITSEVAL)}

FITSEVAL is a sketch planning-level ITS evaluation tool that was developed for the Florida Department of Transportation (FDOT) by FIU researchers. The tool works within the Florida Standard Urban Transportation Modeling Structure (FSUTMS)/Cube environment. It can be used to estimate the benefits and costs of various types of ITS deployment, including Ramp Metering, Incident Management Systems, Highway 
Advisory Radio (HAR) and Dynamic Message Signs (DMS), Advanced Travel Information Systems (ATIS), Managed Lane, Signal Control, Emergency Vehicle Signal Preemption, Smart Work Zone, Road Weather Information Systems, Transit Vehicle Signal Preemption, Transit Security Systems, Transit Information Systems and Transit Electronic Payment Systems.

The evaluation methodology implemented in FITSEVAL varies with the type of ITS deployments. For some types of deployments, it is just a postprocessor of the demand models. For other demands types of deployments, the evaluation requires running assignment steps. The output of the FITSEVAL tool includes the impacts of ITS on performance measures related to mobility, safety, fuel consumption, emission and other measures. FITSEVAL also outputs the benefits and costs in dollar values of ITS and the resulting benefit/cost ratios. These outputs can be used to assess the ITS deployment, prioritize alternatives, and support long-range planning. In a recent assessment by the University of Virginia, twelve different existing tools were evaluated, and FITSEVAL was recommended for use in Virginia (Hadi and Xiao, 2015).

\subsubsection{TOPS-BC}

TOPS-BC (Sallman et al., 2013) is an Excel-based tool that is designed to support practitioners in conducting the benefit and cost analyses of ITS. Thus, it is a much simpler tool than the FITSEVAL tool. It has four main capabilities: 1) Provides information regarding the impacts associated with prior deployments of Transportation System Management and Operations (TSM\&O) strategies; 2) Recommends methods and tools at 
different analysis levels of benefit and cost for these strategies; 3) Estimates the life-cycle costs, replacement costs, and annualized costs based on default cost data. The life-cycle costs include capital costs, as well as the soft costs required for design, installation, operations and maintenance of the equipment. The replacement costs are the periodic costs of replacing/redeploying system equipment. The annualized costs represent the average annual expenditures that would be expected in order to deploy, operate, and, maintain the TSM\&O strategies; and 4) Estimates the benefits for particular TSM\&O strategies. Default impact values and parameters are recommended in this tool. The TOPS-BC tool can evaluate TSM\&O strategies, including traveler information (highway advisory radio (HAR), dynamic message signs (DMS), and pre-trip travel information), ramp metering systems (central control, traffic actuated, preset timing), incident management systems, signal control, emergency vehicle signal preemption, ATDM speed harmonization, employer based traveler demand management, ATDM hard shoulder running, ATDM high occupancy toll Lanes, road weather management, work zone and supporting strategies (traffic management center, loop detection, CCTV).

\subsubsection{Macroscopic Models}

Macroscopic models can be used with and without traffic assignment. The tools developed based on HCM facility procedures can be considered macroscopic models. Another example of these models are regional demand forecasting models, such as the traffic models incorporated in the Bureau of Public Road (BPR) and Akcelik equations. These models calculate the travel time or speed as functions of segment demand to capacity 
ratio. In general, these equations do not reflect queuing and spillback effects. More advanced macroscopic simulation models are applied in dynamic traffic assignment (DTA) tools such as the model incorporated in VISUM.

\subsubsection{Highway Capacity Manual (HCM) Based Tools}

This section describes modeling tools that are based on freeway and urban street facility producers. These can be considered macroscopic tools.

\subsection{STREETVAL}

STREETVAL was developed as a Microsoft Excel sheet using the Visual-Basicfor-Applications (VBA) programming language. STREETVAL can be used to quantify the mobility and reliability levels of service for an urban street facility. The tool implements the urban street facility procedure of the HCM. (Bonneson 2014).

\subsection{FREEVAL}

FREEVAL incorporates HCM freeway facility procedures. It was originally developed as a Microsoft Excel-based tool, with most computations embedded in Visual Basic modules. However, a newer version of FREEVAL (FREEVAL-2015E) was developed in the JAVA programming language. The user can analyze a freeway facility of up to 70 analysis segments and for up to twenty-four 15 -minute time intervals. In FREEVAL, the freeway facilities are divided into different types of segments, including basic, merge, diverge, and weaving segments. An extension of FREEVAL provides the estimation of travel time reliability. In the extension, traffic demand and constructions are 
modeled deterministically, while the occurrence of incidents and weather are modeled using a stochastic approach. Undersaturated and oversaturated conditions can be analyzed by this tool. For undersaturated conditions, roadway segments are analyzed independently. Based on the segment type, the related HCM procedure is applied to determine the segment speed, capacity, and in turn its density and the level of service. When traffic is in oversaturated conditions, the freeway facility is examined as a node-link system utilizing a cell transmission model-based algorithm, which allows accounting for queue accumulation and dissipation over multiple segments and periods. FREEVAL output performance measures include average speed, density, and LOS for each segment and each time interval.

\subsubsection{VISUM}

The traffic flow model used in combination with the DTA in VISUM is a macroscopic model. VISUM is a demand forecasting tool developed by PTV, which includes both static assignment and DTA modules. The DTA model assigns timedependent O-D matrices onto the network using the Dynamic User Equilibrium (DUE) of travelers. The VISUM traffic model considers spillback by assuming that each link can be influenced by two time-varying capacities at the beginning and end of the link, which are called "entry capacity" and "exit capacity". The exit capacity is the capacity of the subject link as coded by the user and is influenced by the signal timing at the downstream intersection. The entry capacity is used to define the effects of queues propagating backwards on the links downstream, which can influence the upstream links, due to 
spillback effecting link. VISUM has an Origin-Destination (O-D) Matrix Estimation (ODME) tool based on initial O-D matrices and count data. An iterative method is applied to adjust the initial O-D matrix cells to attain better matching of observed and simulated volumes.

\subsubsection{Mesoscopic models}

Mesoscopic models are developed to provide a more detailed traffic representation than macroscopic models. They are capable of modeling larger sub-networks and possibly small to midsize regional networks. Mesoscopic simulation models generate and track individual vehicles or packets of vehicles. However, it moves these vehicles according to macroscopic relationships. These models utilize the mesoscopic relationships between speed, density, and flow and consider queuing and spillback due to the subject link capacity and downstream link queuing capacity. Mesoscopic models provide less detail than microscopic simulation models. Nevertheless, they provide much better computational efficiency, which is particularly important for simulation-based DTA. Examples of mesoscopic simulation models are Dynasmart-P, DynusT, DTALite, Direct, Dynameq and Cube Avenue. It should be noted that the first three tools are all based on the same original models developed as part of the Dynasmart project development effort. Though a significant amount of data is still essential to develop, calibrate, and validate mesoscopic traffic simulation models, the required calibration and validation effort is considerably lower than microscopic simulation tools. 


\subsubsection{DYNASMART}

The dynamic Network Assignment Simulation Model for Advanced Road Telematics-Planning, (Dynasmart-P) has been developed by Dr. Mahmassani's group since the early 1990s. (Murray et al., 2001).The DYNASMART simulation model uses the Modified Greenshields relationship, which estimates speed as a function of density with consideration of link capacity and downstream link queuing capacity constraints. There are two methods for inputting demands into the Dynasmart-P tool. The first method is to use time-variant O-D matrices among origin-destination zones. The second, referred to as the vehicle loading method, requires inputting the origin and destination of each vehicle with or without their corresponding travel paths. With this model, the user is assign activitybased demands. Dynasmart-P can model different intersection controls including no control, yield signs, stop signs, pre-timed signals, and actuated signals. It allows signal offsets as inputs. Thus, it allows modeling coordination.

\subsubsection{DynusT}

DYNamic Urban Systems for Transportation (DynusT) was developed at the University of Arizona by Chiu et al. (2012). Based on the Dynasmart model, discussed earlier. One of the first improvements of DynusT was to enhance the Dynasmart mesoscopic traffic simulation model by implementing what is referred to as the Anisotropic Mesoscopic Simulation (AMS) .With this model, vehicle's prevailing speed is not assumed to be the same over all segments of a link, but it is assumed to be influenced only by the vehicles in front of it, in a region called as the Speed Influencing Region (SIR). The 
influence of traffic downstream of a vehicle speed decreases with increased distance. This model shows more realistic representation of traffic flow compared to the original Dynasmart model (Chiu et al., 2012). DynusT also includes a gap-based DUE method that has been implemented as the default assignment algorithm instead of originally implemented the Method of Successive Averages (MSA) method because it has much better convergence and computational efficiency compared to MSA (Chiu and Bustillos 2009).

\subsubsection{Cube Avenue}

Cube Avenue is a mesoscopic simulation-based DTA extension of the Cube Voyager demand forecasting environment (Citilabs 2011). One of advantages of Cube Avenue for regions that are using the Cube modeling environment is that it has the same data format and scripting language as the Cube Voyager tool used in these regions. With the Cube Avenue, vehicles are clustered into homogenous "packets" and simulated as they move through the network. Macroscopic speed-flow relationships, such as the BPR and the Akcelik volume-delay relationships are uses for the movement of the packets. The subject link capacity and the downstream link queuing storage capacity constrains the movements. The user equilibrium assignment utilizes the MSA method.

\subsubsection{DTALite}

DTALite has been developed by Zhou et al (2010). it is an open-source DTA package. DTALite is a mesoscopic simulation-based DTA package working in 
combination with the Network Explorer for Traffic Analysis (NEXTA) graphical user interface. The tool is based on the Dynasmart method. The software architecture of DTALite aims to integrate modeling and visualization capabilities using a modularized design.

The simulation and traffic assignment modeling in DTALite reiterate to either capture day-to-day user response or find steady-state equilibrium conditions. DTALite's output data can be visualized using the NEXTA user interface. DTALite has been used recently in several pilot and research project sponsored by FHWA and the SHRP2 program (Nevers et al., 2013).

DTALite keeps track of each link cumulative flow counts entering and leaving. The cumulative flow counts are useful when applied with queueing models. DTAlite uses a point queue model, by considering the spatial storage capacity constraints that can capture queue spillbacks (Zhou and Taylor 2014). The queue model uses linkspecific jam densities to estimate the physical space availability at the downstream links when no traffic is moving. In addition, DTAlite uses the cumulative arrival and departure curves to track the kinematic waves. The Newell's flow model is also used in the software to represent traffic congestion propagation and capture shockwaves as the result of the bottleneck capacity constraints. However, this procedure cannot account for the variation in midblock incident locations and may not account for additional inefficiencies in upstream and downstream intersection operations, as described later in this document. 
Different node types such as origin, merge, and signalized intersections are defined. In the case of signalized intersections, the model estimates the link outflow capacity by considering the influence of traffic signals. The outflow capacity for incoming links at signalized intersections is allocated using the equation below:

$$
\operatorname{Cap}^{\text {Out }}(l, t)=q^{\text {saturation }}(l, t) \times \frac{E g(n)}{C(n)}
$$

where

$$
\begin{array}{ll}
\operatorname{Cap}^{\text {Out }}(l, t) & =\text { outflow capacity of link } 1, \\
q^{\text {saturation }}(l, t) & =\text { saturation flow rate on link } 1, \\
E G(n) & =\text { effective green time, and } \\
C(n) & =\text { cycle time for upstream node } \mathrm{n} .
\end{array}
$$

An incident occurring at a mid-block location can decrease the upstream intersection throughput, if the queue from the incident spillbacks to the upstream link. The upstream link capacity is estimated by using a model estimates the link ( $l$ ) outflow capacity at time $\mathrm{t}$ as function of its maximum saturation flow $q^{\max }(l, t)$ and its upstream link inflow capacity $\operatorname{Cap}^{i n}(1+1, t)$ as shown in the equation (2-2).

$$
\operatorname{Cap}^{\text {out }}(l, t)=\min \left\{q^{\max }(l, t), \operatorname{Cap}^{\text {in }}(l+1, t)\right\}
$$




\subsubsection{Dynameq}

Dynameq is a simulation-based DTA environment, developed by INRO (INRO 2016). The developer of the EMM2 demand forecasting model. One of the strengths of Dynameq is its more detailed simulation models computed to mesoscopic models. It capable to model lane-by-lane traffic, that is not possible with existing mesoscopic simulation models. The simulation model is considered as event-based simulation. Eventbased simulation is much more computationally efficient compared to time-interval based simulation. However, it has a lower fidelity, because it only updates the simulated network once changes occur to the network conditions. The, propagation of traffic delays can be modeled in Dynameq with a simplified car-following model, utilizing a macroscopic triangular flow-density traffic flow relationship. Therefore, Dynameq can be counted as a high fidelity mesoscopic simulation model.

The DTA in Dynameq is DUE assignment, iterating between finding timedependent path flows and determining the corresponding path travel times. The successive Averages method (MSA) is used to assign vehicles to paths. Noted, developers have also tested more productive and enhancing converging methods of assignment (Mahut et al., 2004).

\subsubsection{Microscopic Models}

Microscopic simulation tools evaluate and analyze the network at much more detailed levels than macroscopic models. Microscopic flow models such as car-following, lane-changing, and gap acceptance are used in microscopic simulation tools to model the 
movements of individual vehicles. With these models, vehicles are tracked through the network over small time intervals, as short as one tenth of a second. However, microscopic simulation tools require more resources and efforts to use the network. In general, it is used for smaller networks. In particular, the calibration and validation of microscopic simulation models, demands significantly more time than calibration mesoscopic models. Examples of microscopic simulation models are TransModeler, VISSIM, PARAMICS, CORSIM, and AIMSUN.

Hybrid simulation models combine mesoscopic simulation with microscopic simulation. Hybrid simulation models combine the strengths of both modeling approaches and address their individual weaknesses. The hybrid mesoscopic-microscopic model can apply microscopic simulation to areas that require specific attention, while simulating a surrounding network in lesser details with a mesoscopic model. An important feature in developing a hybrid model is the implementation and execution of consistent interfaces between the mesoscopic and microscopic simulation components of the simulation model. The consistency considerations include the structural compatibility in terms of modeling traffic flows in the two models (Burghout 2004; PTV 2015). The recent versions of VISSIM, TrasnModeler and AIMSUN are considered as Hybrid simulation tools.

\subsubsection{AIMSUN}

The TSS-Transportation Simulation Systems Company in Spain is the developer of the Advanced Interactive Microscopic Simulator for Urban and Non-Urban Networks (AIMSUN) microsimulation tool. AIMSUN is considered an integrated traffic modeling 
software fusing the travel demand model, static and dynamic traffic assignment with mesoscopic, microscopic and hybrid meso/micro simulation (TSS 2015). AIMSUN has been particularly recommended for modeling ITS applications. Stochastic and discrete route choice models and dynamic user equilibrium are offered in AIMSUN that can be considered with microscopic, mesoscopic and microscopic simulations. The user of the, dynamic traffic assignment in AIMSUN can select from three types of shortest paths including, user predefined paths, calculated shortest path tree based on initial defaults or user defined costs, and calculated shortest path tree based on statistical data collected from the simulation. In addition, AIMSUN has a powerful tool to model ITS applications. AIMSUN utilizes two facilities to model ITS applications and their effects on driver behavior. The first is the Microscopic Simulator Software Development Kit (microSDK), allowing users to override default behavioral models (car-following, lane-changing, etc.), and the second is the AIMSUN Platform Software Development Kit (platformSDK) for developing new interface for ITS applications, which gives the power to users to implement their own menus and dialogs and add new classes of objects to the data base (TSS 2015).

\subsubsection{TransModeler}

TransModeler is a microscopic simulation-based traffic assignment tool developed by Caliper. One of the interesting features of TransModeler is its capability to model parts of the network at the microscopic level and parts of the network at the mesoscopic and/or macroscopic simulation level in the same run. This is a type of Multi-resolution simulation that can be used to reduce the resources required to model larger networks (Caliper 2015). 


\subsubsection{VISSIM}

The Verkehr In Stadten Simulation (VISSIM) tool has been developed by the PTV Group in Germany. The first version of VISSIM was released in 1993. VISSIM utilizes a link-connector structure, which is different from most existing simulation models, which utilize link-node configurations. This capability allows for increasing accuracy and flexibility of modeling. Although coding of the link-connector model is more complex than coding the link-node models, it has been simplified in more recent versions of the software. Another interesting feature of VISSIM is its ability to display results in 3-D animations, plus a feature which allows viewing from a selected driver's perspective. VISSIM has also a powerful programing extension, allowing modelers and users to program advanced managements and pricing strategies. VISSIM allows inputting traffic demands as predetermined turning movement proportion, partial routes movement or O-D Matrices, if the DTA model is to be exercised (PTV 2015). VISSIM 8 is latest version of the VISSIM software. VISSIM 8 has the ability to be a hybrid mesoscopic-microscopic model (PTV 2015).

\subsubsection{CORSIM}

CORSIM is a microscopic traffic simulation software package for arterial and freeway systems developed by the FHWA in the early 1990 based on two models developed previously (FRESIM for freeway and NETSIM for arterials). CORSIM does not have DTA model and requires the users to input turning movement counts. CORSIM and TRANSYT-7F, signal optimization program are now offered as one combined 
product. TRANSYT-7F improves the traffic signal analysis functionality of CORSIM by importing CORSIM files and optimize their signal timing, generating input files for CORSIM, then compute the performance of the signal control. One of the useful features of CORSIM is its ability to model incidents directly, considering the rubbernecking effect and the locations where drivers start changing lanes a head of the incidents. The rubbernecking modeling CORSIM increases the time headway between vehicles reflecting the driver behavior when observing the incident. (Alvarez and Hadi 2010) (Alvarez and Hadi 2010) (Alvarez and Hadi 2010) (Alvarez and Hadi 2010) (Alvarez and Hadi 2010) (Alvarez and Hadi 2010) (Alvarez and Hadi 2010)

Paramics is traffic microsimulation tool developed in Scotland Ltd. Paramic has been used to model ITS alternatives including Variable speed limits (VSL), high occupancy tolling (HOT), vehicle actuated signals, incident response, HOV lanes, dynamic lane control, route choice updates, roadside message signs, and car parking signs. Paramic has a number of assignment types including stochastic assignment, dynamic assignment, and the preferred route assignment (Paramics 2015).

\subsubsection{SimTraffic}

Synchro/SimTraffic is a software package developed by Trafficware for modeling and optimizing traffic signal timings. SimTraffic is a micro-simulation tool that utilized with the Synchro signal optimization tool can optimize signal timings of signalized facilities (Trafficware 2015). SimTraffic has less features compared to other simulation tools. SimTraffic structure is very similar to CORSIM, which is a link-node model. Also, 
SimTraffic uses many of the same driver behavior and vehicle performance parameters used in CORSIM. However, it has been reported that the car-following algorithm is the greatest difference between CORSIM and SimTraffic. SimTraffic incorporates a more user-friendly interface that greatly eases network coding requirements and interpreting outputs (Jone et al., 2004).

\subsection{Incident Management}

Incident management is an important component of Transportation System Management and Operations (TSM\&O), providing significant benefits in terms of travel time, travel time reliability, emission, fuel consumption, safety, and other performance measures of transportation systems (Massahi et al., 2017; Baqersad et al. 2017; Ahmad et al. 2016). Estimating the impacts of incidents and incident management strategies allows traffic management agencies to determine the needs for various incident management strategies and technologies and to justify the decisions to invest in their programs (Fesharaki 2016). This justification is critical when requesting additional funds for future activities of the programs. Traffic Incident Management is a coordinated and planed programs to detect, respond, and clear traffic incidents (Knoop et al., 2009). Incident management centers cooperate and collaborate with multiple agencies, such as government officials, fire and rescue, police, highway patrol, emergency medical services, hazardous material crews, and towing services in order to facilitate nonrecurring congestion problems on freeway and arterial networks (Bhide 2005). 
Incident management components can be described as a collection of activities that take place during four main stages: detection, response, clearing, and recovery. The main elements of incident management include the incident detection, incident verification, response selection, incident removal, traffic management, and the provision of traveler information. Each of these elements, separately and cooperatively, impacts the efficiency and success of the incident management strategies. Thus, well-organized incident management systems or plans are vital for the effectiveness of the systems (Bhide 2005). The first part of this section will review incident duration reduction because of implementation of incident management. Also, other related incident duration reduction benefits such as reduction of fuel consumption and pollutants amount will be reviewed. Second part, provides the necessary information about diversion rate because of incident and incident management role. Next part gives overviews about the impacts of incident management on safety and incidents' frequency. The last part discusses different tools and software abilities to model and implement the incident and incident management.

\subsubsection{Impact of Incident Management on Incident Duration}

Reducing incident duration and minimizing its negative impacts is one of crucial objectives of incident management strategies. An evaluation of the CHART (Coordinated Highways Action Response Team) program conducted in the year 2006 by University of Maryland, estimated that the average incident duration between 2000 and 2006, was 33 minutes with CHART and 77 minutes without it (in 1999). In a more recent evaluation of the CHART program it was fond, the average incident duration was estimated to be about 
22.92 minutes with CHART and 32.45 minutes without it (Young and Tarnoff, 2008). Khattak et al. (2007) conducted a study on the effect of the Chart program on incident delay and emission in Hanover, Maryland. The average incident duration was founded to be shortened from 33 minutes to 29 minutes over the same period by running the CHART program (Chang and Rochon, 2006).

Improving incident Management program to a program that includes detection, verification in Atlanta, Georgia, reduced the average time between the first report and incident verification by $74 \%$. Improving incident detection and management tech equipment and using CCTV cameras for incident detection and verification in San Antonio, improved the response time by $20 \%$ (21\% reduction for major incidents and $19 \%$ for minor incidents). An incident management implementation in Brooklyn, NY reduced the incident clearance average time by $66 \%$. Some agencies put efforts to improved interagency communications for incident detection and verification to reduce the incident response time and consequently its clearance time. For instance, the automatic tow truck dispatch program in Minneapolis/St. Paul (Highway Helper) decreased the incident response and removal times by 20 minutes (85\% improvement) (Systematics 2013).

Several researches have evaluated the service patrol system role to reduce incident duration. The Atlanta's NAVIGATOR system, reduced the service patrol truck dispatching time from 21 minutes to 10 minutes (52\%). Consequently, the average incident clearance was dropped from 26 minutes to 20 minutes $(23 \%)$. It was estimated that this program 
reduced $5.775 \mathrm{~kg}$ of hydrocarbons $(\mathrm{HC}), 75.58 \mathrm{~kg}$ of carbon monoxide (CO) and $8.059 \mathrm{~kg}$ of nitrous oxides $\mathrm{NO}_{\mathrm{x}}$ per incident.

On the I-880 freeway in San Francisco, CA; service patrol implementation decreased the average response time from 28.9 minutes to 18.4 minutes (36\%) and the clearance time from 9.6 minutes to 8.1 minutes $(16 \%)$. The total delay saving per assisted breakdown was 42.4 vehicle-hours and the savings per assisted accident was 20.3 vehiclehours per incident (Skabardonis et al., 1995).

Another study reported that service patrol implementation dropped the total duration of incident by 16.5 and 10.5 minutes in Houston, TX and Denver, CO respectively. Studies in Minnesota (MNDOT 2004)and Maryland (Sorensen et al., 2008), reported that Freeway service patrol (FSP) reduced the average incident duration by well over $50 \%$ (Sorensen et al., 2008). In Pittsburgh, the service patrol program reduced response time to incidents from 17 to 8.7 minutes. In Oregon, the service patrol implementation shortened the delay-causing incidents by approximately $30 \%$ on two lane Highway and $17 \%$ on Interstate. In Northern Virginia, response vehicle services; reduced the duration for all incidents by 2 to 5 minutes due to using cell phone in response vehicles, 2 to 5 minutes due to CAD screens in response vehicles, and 4 to 7 minutes due to GPS location in response vehicles (Systematics 2013).

The ITS Deployment Analysis System (IDAS) uses default values for incident duration reduction of $9 \%$ for incident detection and verification systems, $39 \%$ for incident response and management systems and 51\% when implementing a combination of these 
systems (Hadi et al., 2008). It has been reported that TrsnsGuide incident detection and response system in Houston dropped the average incident duration by $30 \%$ and Georgia Navigator system reduced incident clearance time by an average of 23 minutes and the incident response time by 30\% (Economic Development Research Group, Inc 2013). The Maryland (CHART) incident management program reduced the blockage duration from incidents by 36\% (Economic Development Research Group, Inc 2013).

The Florida DOT, District IV, evaluated the impacts of the incident management actives in Broward County with the assumption the incident duration is reduced by $18 \%$ (FDOT 2006).

As described above impacts of incident management on incident duration and the associated mobility measures have been widely investigated for freeway facilities (Khazraeian et al., 2016). Since, arterial incident management activates here not been considered widely in the past, there is lack of research and data to estimate its impacts on network performance. Dia and Cottman (2006) conducted a research to evaluate arterial incident management impacts. Based on their research, incident management strategy can reduce the duration of an incident blocking all lanes from 30 to 15 minutes. The environmental benefits demonstrated a $22 \%$ reduction in fuel consumption rate and $20 \%$ reduction in $\mathrm{CO}_{2}$. A study used to assess the benefits of incident management in Palm Beach County classified the accidents into three classes: major incidents (incident duration of 30 minutes or more), intermediate incidents (incident duration is 15 minutes to 30 minutes), and minor incidents (incident durations of less than 15 minutes). The study is 
assumed that, if the arterial incident management is active, the incident average durations are reduced by $20 \%, 12 \%$, and $5 \%$ for major, intermediate and minor incidents respectively.

\subsubsection{Impact of Incident Management on Diversion Rate}

Incident information is disseminated using a variety of platforms such as highway dynamic message signs (DMS), highway advisory radio (HAR), traveler information telephone systems, 511 phone services, smart cellphone applications, radio stations, social media, and websites. Travelers recurring information from these platforms may adjust their route to avoid delays. The estimation of diversion rates is needed to assess the benefits of the information dissemination, the impacts on alternative routes, and the needs for updated signal control plans and other traffic management strategies on these routes during incident conditions.

Several European field studies have found that the diversion rate because of DMS messages, range from $27 \%$ to $44 \%$.Knoop et al. (2009) found that $50 \%$ of travelers take alternative routes if they faced with major incidents (Tarry and Graham 1995). Kang and Gillen (1999) came up with 10 categories of demand data during the peak period, the diversion rate was classified into 5 cases from $3 \%$ to $15 \%$. Hadi et al. (2008) developed a liner model, which estimates the saving in delay based the on diversion rate with a default maximum diversion rate was selected as $40 \%$ based on dissection with FDOT state. The incident management programs in Brooklyn, NY on the Gowanus 
Expressway reduced the average incident clearance time from 1.5 hours to 31 minutes (Michalopoulos and Samartin 1998; Alluri et al., 2017).

\subsubsection{Impact of Incident Management on Safety}

In Atlanta, Georgia the evaluation of the NaviGAtor incident management program indicated a reduction in secondary crashes by $69 \%$ (from 676 to 210 in one year) (Carson 2009). The Maryland, CHART program accomplished $15 \%$ to $38 \%$ reduction in all secondary crashes, $4 \%$ to $30 \%$ reduction in rear-end secondary crashes, and $21 \%$ to $43 \%$ reduction in severe secondary crashes. IDAS assumed that $21 \%$ of fatalities are shifted to injuries because of reduction in incident response time due to incident management strategies (Systematics 2013).

\subsubsection{Incident and Incident Management Modeling in Existing Tools}

In the absence of field measurements of incident delays; queuing theory, shock wave, and simulation analyses have been used to assess incident delays. Microscopic traffic simulation is a powerful method to estimate the impacts of incident and incident management (Gomes et al., 2004; McCandless, 2007) .However, the use of simulation models is expensive in terms of data collection, model input preparation, and calibration, particularly when the incident management systems need to be assessed at the regional levels and when the stochastic nature of incident attributes and locations need to be considered in the analysis. 
The CORSIM microscopic simulation program is unique among simulation models in that it has a specific frame to model incident on freeways. However there is no such frame to model incident on arterial facilities in CORSIM. CORSIM is capable to drop the capacity in the vicinity of a freeway incident, that can be fine-tuning using a rubberneck factor and the warning sign location (Yang et al., 2008). In other simulation models such as AIMSUN and VISSIM, there is no specific features to model incidents. Incidents should be model by introducing events which have the same operational effects as lane-blockage incident such as specifying stopped bus with bus dwell time equal to incident duration and fine-tuning the parameters of the open lanes to produce the expected drops in capacity (May 1990; Hadi et al., 2007).

Red signal and parking space have also been used to emulate incidents (Hadi et al., 2007; Zhou and Tian 2012). Hadi et al. (2007) found that it is important to use the speed reduction area on the adjacent lanes since without using this area; the reduction in capacity due to an incident lane blockage in VISSIM was much lower than the HCM estimates of the capacity reductions. Hong et al. (2013)set up a red signal at the incident lane to simulate the incident and used the reduced speed area to adjust the capacity drop in the adjacent open lanes. The signal turns red once the incident occurs, turns to green when the incident is cleared. Avetisyan et al. (2014) used the "Add vehicle" function, within the VISSIM's COM interface, to put a vehicle with zero speed at the time and location of the incident and inserted reduced speed area for the adjacent lanes. In the above studies, the length of "reduce speed area" in the vicinity lanes of the incident was modified by trial and error to 
achieve the expected drop in capacity due to the freeway incident. The incident management can be assessed by reducing the incident duration before runs.

In the TOPS-BC spreadsheet-based selection planning toll, traffic incident management (TIM) is evaluated in terms of two main benefits: travel time reliability improvement and fatality crash reduction. The calculation of the additional TIM benefits including the reductions in secondary crashes and fuel use are optional. The improvement in travel time reliability is calculated as the reduction in incident-related delays, which is a function of the percentage reduction in incident response time. A certain percentage of fatality crashes are considered to be changed to injury crashes with the quick response of TIM in the safety benefit calculation. For the optional analysis of secondary crashes and fuel use, TOPS-BC requires the users to input the corresponding reduction factors (Sallman et al., 2013).

The FITSEVAL tool assumes that incident management reduces the incident duration and consequently the incident delays. The reduction in incident delay is calculated based on queuing analysis with and without incident management. The time savings due to vehicle diversion during the incidents are considered in this evaluation methodology. The diversion rate is set as a function of the estimated saved delays. This methodology also assumes that $21 \%$ of fatalities are shifted to injuries due to faster incident detection, verification, and response of incident management systems. In addition, an additional reduction factor of $2.8 \%$ is used to account for the impact of incident management on accidents. The emissions and fuel consumptions with and without incident management 
are estimated based on the speeds of queued and non-queued vehicles and the vehicle-miles in queue utilizing the Environmental Protection Agency (EPA) Mobile6 program simulation tool was developed before the resales of the EPA MOVES program. The tool assumes that for the same incident and traffic conditions, the incident delays on the arterials are 1.25 higher on signalized arterials compared to uninterrupted facilities (Yang et al., 2008).

\subsection{Signal Control Strategies and Their Effects on Network}

Signal control strategies and plans play a crucial role in improving network performance and mitigating certain types of recurring and non-recurring congestion. A primary goal of adaptive signal control, readjusting signal retiming and condition-response signal is to minimize the network overall delay, which provides high benefits in terms of mobility, safety, sustainability and reliability during recurring and non-recurring congestion. The first two parts of this section review adaptive signal control and signal retiming strategies and their impacts on transportation network performance. Next, this will review the benefits of these strategies and plans. This is followed by an overview of adaptive signal control and signal retiming benefits during incident. Finally, a review is presented of section, weather-response signal controls and their influences on system performance. 


\subsubsection{Adaptive Signal Control}

In the United States, many adaptive control systems have been deployed, but adaptive systems still control less than $1 \%$ of all the traffic signals in the nation. As detection and communications technology improves, adaptive systems are becoming more popular and effective. This section provides a brief description of some systems that have been deployed in the U.S. over the last 20 years (Koonce et al., 2008). Adaptive signal control systems use algorithms that select traffic signal timing parameters based on current traffic conditions, demand, and system capacity. The adaptive control software adjusts traffic signal splits, offsets, phase lengths, and in some cases phase sequences to minimize delay and reduce the number of stops.

An Evaluation of the impacting on Adaptive Traffic Control System (ATCS) in Los Angeles was conducted by the floating car method at the selected segments over four days during daylight hours under clear, dry weather conditions. The assessment results indicated that the ATCS decreased travel time by $12.7 \%$, reduced average stops by 31.0 $\%$, and lowered average delay by $21.4 \%$ (Banerjee and Frances 2001). An adaptive signal timing project in Gresham, Oregon reduced the average travel times by $10 \%$ and saved over 74,000 gallons of fuel every year (Bond et al., 2013). Sussman (2000) reported that adaptive traffic signal control systems decreased travel times by 13 to $25 \%$ in Los Angeles, Broward County, and Newark based on a review of past study. The study indicates that the ATCS improved coordination and reduced the number of stops by 28 to $41 \%$ (Sussman 2000). An evaluation of adaptive signal control of 12 intersections on Route 291 in the 
Lee's Summit, Missouri indicated that the average travel times decreased on the mainline up $39 \%$ depending on the time of day and direction of travel. The number of vehicle stops decreased by $17 \%$ to $95 \%$ per trip. The average vehicle speeds improved 5 to 10 mile per hour most of the time periods. The impacts on fuel consumption ranged between $4.5 \%$ increase and a $21.4 \%$ decrease. Changes to pollutants $\left(\mathrm{HC}, \mathrm{CO}\right.$, and $\left.\mathrm{NO}_{\mathrm{x}}\right)$ emission varied from a $9 \%$ increase to a decrease of $50 \%$ (Hutton et al., 2010). Adaptive signal control systems installed on two corridors in Colorado improved weekday travel times 6 to $9 \%$, increased weekday average speed 7 to $11 \%$ and decreased weekday stopped delay 13 to $15 \%$ (Sprague and Archambeau 2012). An adaptive signal control system in Oakland County, Michigan reduced travel time by $7 \%$ in the morning peak and $8.6 \%$ during evening peak periods. Off peak and non-peak direction travel times were also improved by 6.6 to $31.8 \%$ (Abdel-Rahim et al., 1998).

University of Detroit Mercy, study found that the total crashes per mile per year decreased by $28.8 \%$ between 1999-2001 (before period) and 2003-2008 (after period)(Dutta et al., 2010). It was also reported that deploying advanced traffic signal controllers (ASTC) and an adaptive decision support system for 110 blocks of New York City resulted in a $10 \%$ reduction in travel times (Izzy 2012).

\subsubsection{Time of Day Signal Control Retiming}

Optimizing traffic signal timing is a high-benefit and low cost strategies to reducing congestion. Signal timing strategies try to minimize stops, delays, fuel consumption and air pollution emissions and maximize the traffic progression through the system. Previous 
studies showed that traffic signal retiming strategies result in reductions in travel time and delay between 5 to $20 \%$ and fuel savings of 10 to $15 \%$.

Halkias and Schauer (2004) conducted a research to assess the benefits of signal timing optimization, for 154 intersections, in Syracuse, NY. These optimizations reduced the number of stops by $15.7 \%$, travel time by $16.7 \%$, and delay by $18.8 \%$, resulting in $13.8 \%$ decline in fuel consumption, and a $13 \%$ reduction in vehicle emissions and noise pollution. Their research on retiming of 640 traffic signals in Oakland County, MI shows reductions between 1.7 and $2.5 \%$ in Carbon monoxide, 1.9 to $3.5 \%$ in Nitrogen oxide, and 2.7 to $4.2 \%$ reduction in hydrocarbon (Halkias and Schauer 2004). The Texas Traffic Light Synchronization program, that involved updating the traffic signal control equipment and signal timing reduced delays by $23 \%$ (Gordon et al., 1996). Harris (2005) led a study to evaluate the benefits of retiming traffic signals in several U.S. and Canadian cities. The study reported that the traffic light synchronization program in Texas reduced delay by $24.6 \%$, lowered travel time by $14 \%$, and reduced fuel consumption by $9.1 \%$. Traffic signal retiming at 11 intersections on U.S. Route 1 in St. Augustine, Florida reduced delay by $36 \%$, and lowered travel time by $10 \%$. The program resulted in an annual fuel savings of 26,000 gallons. A retiming project for eight intersections on State Route 26 in Gainesville, Florida reduced the average delay by $94 \%$ and saved 3,300 gallons in fuel consumption annually. Travel time was shortened by $7 \%$ in Burlington, Canada because of signal retiming at 62 intersections; also, fuel consumption was decreased by $6 \%$. In Montgomery County, traffic signal retiming lowered delay by $13 \%$ and reduced fuel 
consumption by $2 \%$. The Fuel Efficient Traffic Signal Management (FETSIM) Program in California deceased delay by $15 \%$ and fuel consumption by $8.6 \%$ (Harris 2005). The traffic signal retiming program at 50 intersections in Lee County, Florida resulted in a 23 $\%$ annual reduction in travel delays, causing $\$ 15,300,000$ in travel time savings. The program also resulted in $\$ 2,000,000$ per year in fuel savings. The effort also decreased vehicle emissions by $19 \%$, resulting in an equivalent to $\$ 124,000$ environmental benefits (ITS International 2012). In Tysons Corner, VA signal timing optimization resulted in a 9 $\%$ reduction in fuel consumption(White 2000).

The Southwestern Pennsylvania Commission's (SPC) Regional Traffic Signal System Program optimized 7 intersections along the Robinson Town Centre Boulevard/Summit Drive corridor of the program. The evaluation results of program indicated that average travel times were shortened by $6 \%$, average stops lowered by $6 \%$, and average signal delay decreased by $16 \%$. First year benefits indicated a benefit-cost ratio of $57: 1$, a reduction of 78,480 vehicle hours in travel time, a reduction of 75,709 gallons in fuel consumption, a reduction of 7,606 $\mathrm{kg}$ in total pollutant emissions, and a reduction of 2,258,000 in number of stops (Allegheny 2011). Optimization of signal timing on the arterial network in Syracuse, NY decreased vehicular delay by $14 \%$ to $19 \%$, reduced total stops by $11 \%$ to $16 \%$, and improved average speed by 7 to $17 \%$ (Lin et al., 2012). A study has been done by Day et al. (2014) to evaluate signal retiming project for a 8.7-mile section of US-31 in Kokomo, IN. The evaluation indicated that signal retiming saved 16,322 hours of travel time and reduced 982 tons of $\mathrm{CO}_{2}$ (Day et al., 2014). Jensen 
et al. (2000) conducted a study about the potential impacts of incorporating information on arterial traffic flow into an Advanced Traveler Information System, which would allow motorists to make more informed decisions about whether to divert from a congested freeway to an adjacent arterial volume. The research results showed, the system would reduce vehicle delay by $1.8 \%$ (Jensen et al., 2000).

Six types of signal control can be evaluated in FITSEVAL and traffic signal retiming is one of them. Retiming traffic signals can slow down the deterioration of link travel time. Thus; signal retiming benefits are calculated in terms of reductions in link travel times. A default value of $7.5 \%$ improvement in travel time is used in FITSEVAL with a retiming of existing coordinated signals (Hadi et al., 2008).

\subsubsection{Comparison Adaptive Signal Timing with Retiming Signal Control}

The study compared optimized signal control plan with adaptive signal control in terms of travel time in four cities: Reston, VA; Chicago, IL; Seattle, WA; and Tucson; AZ. Field tests performed in Virginia and Washington during peak periods reported that travel times improved by approximately 5\%, when adaptive signal control was implemented instead of optimized traffic signal timing plan. Simulation results proved that adaptive control can reduce travel time and improve traffic volume by approximately 3-7\%. In Chicago, IL, the RTACL system dramatically enhanced travel times by 12-53 $\%$ and diminished delay up to $100 \%$ along certain corridors within the network (Ghaman et al., 2002). 
Bond et al. (2013) reported that an adaptive signal timing project installed in Gresham, Portland, decreased the average travel times by $10 \%$ and saved over 74,000 gallons of fuel every year.

\subsubsection{Impact of Signal Timing Strategies on the Network Performance during Incident}

Signal timing strategies can play a vital role in mitigating the impacts of nonrecurring congestion. New signal planning can increase the roadway capacity during arterial incidents and diversion due to freeway incidents. A primary goal of signal timing strategies for incident condition would be to give priority to specific movements in order to minimize the overall delay. One way to reach this goal would be to increase or decrease the throughput of traffic at certain intersections by increasing or decreasing the green times for those movements. In addition, modifying signal timing can be combined with traveler information that guide motorists to alternative routes (Turnbull 2005).

The CHART program in Maryland investigated strategies to mitigate the incident impacts. The user is informed of alternate routes or notified to expect delays. The incident detection is integrated with the signal system software to allow preset signal timing plans to be implemented once changes like incidents occur. Based on the provided information, the managers will make decisions on whether a diversion and a change of signal timing is necessary for the parallel arterials. The program evaluation estimated a total delay time reduction of 30 million vehicle-hours, and a total fuel consumption reduction of 5 million gallons. 
The City of Portland coordinated with the Oregon Department of Transportation and Multnomah County to develop the incident management plans along Interstates 5 and 205. Based on point traffic detection and CCTV Camera images, dynamic message boards were programmed to notify and advise the motorists of the incident and possible diversion. The signal timing along the adjacent arterials were modified in order to accommodate the diverted traffic (Outlook 2010).

Birst and Smadi (2000) utilized simulation to determine the impacts of a freeway management system displaying incident warnings on DMS and the use of adaptive signal control to mitigate incident-related congestion, in Fargo, ND. A simulation model indicated that the system can decrease travel times by $18 \%$ and increase speeds by $21 \%$ (Birst and Smadi 2000).

Glassco, et al. (1997) simulated a network of the Detroit Commercial Business District, MI, and found that adaptive signal control of alternative routes around an incident reduced delay by $60 \%$ to $70 \%$ for the affected paths (Glassco et al., 1997).

$\mathrm{Lu}$, et al. (2011) developed an integral procedure to decrease the total delays around the incident site, by designing a signal control optimization model and obtaining the optimal solution. The VISSIM simulation results indicated that their proposed method leads to $39.5 \%$ decrease in delay (Lu et al., 2011). Lin (2002) investigated the impact of an Incident Management System (IMS) implementation for the Interstate 75 corridor in Sarasota, FL, with the alternative route operation associated with an optimal signal coordination timing plan. The reductions of average delay per vehicle was 
estimated to be $5 \%, 15 \%, 22 \%$ and $25 \%$ for incident durations of $40,60,80$ and 100 minutes, respectively.

\subsubsection{Weather-Response Signal Control}

Atmospheric events can decrease the efficiency of traffic signals, therefore, significant benefits are expected from implementing specific signal timing plans during adverse weather (Goodwin 2002). Depending on the region, adverse weather can include heavy rain, thunderstorms, slush, ice, and snow. Adverse weather can reduce visibility and pavement friction, consequently it changes driver behaviors (Sadeghvaziri et al., 2016) Readjusting signal timing plans is expected to mitigate delays due to these effects. Such adjustment would consider the increasing headways between vehicles in inclement weather.

Although, the negative impacts of inclement weather on traffic flow condition is widely acknowledged, there has been limited research to quantify the impacts of inclement weather on traffic flow parameters and signalized intersection operations (AlKaisy and Freedman 2006). Seeherman and Skabardonis (2013) used historical data from 17 urban freeway corridors in California for estimating the proportion of delays associated with rain. Their study showed that $3-25 \%$ of the total delays are because of rain. Lochmueller (1995) investigated the variation of speed and saturation flow in severe weather conditions during winter. The concluded that signal timing optimized for summer is not suitable for winter because of lower speed in winter (Lochmueller 1995). Botha and Kruse (1992) indicated that unfavorable weather conditions decreases saturation flow 
rates along a highway. Some studies showed that snowy weather decreases saturation flow rate by 20\% (Agbolosu-Amison et al., 2004; Agbolosu-Amison et al., 2005). Gillam and Wilhill (1992)showed that wet pavement increases travel delay by $11 \%$ and decreases saturation flow rate by $6 \%$. Maze, Agarwai et al. (2006) found severe rain causes $10 \%$ to $17 \%$ reduction in capacity and $4 \%$ to $11 \%$ reduction in speed. Rakha, Farzaneh et al. (2008) investigated the inclement weather effects on speed, flow and density. They concluded, that these parameters vary as a function of the precipitation type, precipitation intensity, and visibility level. Rämä and Luoma (1997) showed about $10 \%$ to $30 \%$ reduction in the average speed during icy and snowy conditions.

Maki et al. (Maki 1999) conducted a study for the Minnesota Department of Transportation to evaluate the feasibility of implementing a traffic signal timing plan for inclement weather conditions. A signal timing optimization program was used for optimizing signal timing. Inclement conditions were modeled by adjusting the saturation flow rates and the average speeds. It was concluded that the adjusted signal timing resulted in $8 \%$ reduction of signal delay for each vehicle and a $6 \%$ reduction in average stops in comparison with normal signal timing. In Charlotte, NC, traffic signals during inclement bad weather were modified to operate with increased cycle lengths or with peak period timing plans during the off-peak period. This led to a reduction in travel speeds and a decrease in the probability of the severity of crashes (Goodwin and Pisano 2003). Balke and Gopalakrishna (2013) performed a study to implement and evaluate a weather responsive traffic signal timing system in Ogden, UT. They utilized a software tool 
(Purdue Coordination Diagram) to visualize and manage signal performance, and a Traffic Estimation and Prediction System (TrEPS) tool for forecasting traffic conditions and decision support for operators. The weather responsive timing plan reduced the cumulative travel time by $4.3 \%$. The study results showed $11.2 \%$ reduction in the cumulative stop time compared to using the time-of-day plans during the snow event. In addition, the study found that the travel times of cross-street improved by $3 \%$, and the overall cross-street stopped times decreased by $14.5 \%$ (Balke and Gopalakrishna 2013). Abdel-Rahim et al. (2011) investigated the effects of weather-responsive signal timing plans on safety and mobility. Their study results indicated a potential average reduction in rear-end conflicts of approximately $22 \%$ for moderate volume levels and $43 \%$ for high volume levels (Abdel-Rahim et al., 2011).

\subsection{Incorporation Travel Time Reliability}

Reliability measurement allows the assessment of the variability of travel times

of the same time unit (hour, day of week, season) observed for different days and averaged across individual travel times observed within the unit for the same day. A number of studies have been conducted to incorporate travel time reliability into microand meso-simulation models as well as integration in demand and network models. The incorporation of reliability factors into the models can be achieved using one of two methods. The first method is analytical which implicitly treat travel time as a random variable from a distribution, as reflected by the parameters of this distribution. The second method is empirical. With the empirical method, travel time distribution is not 
parameterized analytically. However, it is simulated directly or explicitly through multiple model runs with multiple scenarios (Kwon et al., 2011). Prior studies identified the factors that impacted travel time reliability including: traffic incidents, weather, work zones, fluctuations in demand, special events, traffic control devices, and inadequate base capacity. The evaluation of travel time reliability is critical to the assessment of ATDM strategies. Improving reliability is one of the most important goals of implementing ATDM strategies (Kwon et al., 2011). This section gives general information about reliability, its performance measurements, and source of unreliability. The second part of this section reviews the incorporation of reliability into transportation models. The third and fourth parts will analyze the process for mesoscopic and microscopic models.

\subsubsection{Concept of Reliability}

This section reviews the indices used in travel time reliability estimation and measurement, sources of the unreliability, and recent work on estimating travel time reliability utilizing modeling tools.

\subsubsection{Travel Time Reliability Indices}

Previous studies have identified a number of reliability performance measures and provided recommendations on their suitability for different purposes. Lomax, Schrank et al. (2003), presented three broad categories of reliability performance indicators including: statistical range, buffer time measures, and tardy trip indicators. The study defined a set of six reliability metrics: Buffer Index, failure/on-time measures, Planning Time Index, 90th 
and 80th Percentile Travel Time Index, Skew Statistic, and Misery Index. These reliability measures may be quantifiable at network-level, O-D-level, and path/segment/ link-level. Table 2-2 defines the reliability metrics.

\subsubsection{The Value of Travel Time Reliability}

An important issue associated with modeling travel time reliability is the value of reliability (VOR) (Shams et al., 2016; Hossan et al., 2016; Jin et al., 2017). Travel time has a long history of issues in transportation modeling and analysis. The valuing travel time (VOT) refers to the monetary value that travelers place on reducing their travel times. The VOT value has been related to the wage rates of travelers. In contrast, the VOR is a reliability new concept is defined as monetary value that travelers place on reducing the variability of their travel time (Carrion and Levinson 2012). Economic Development Research Group, Inc (2013) proposed reliability ratio (VOR/VOT), 0.8 and 1.1 for passenger cars and trucks respectively. This ratio can be used to compute the Travel Time Index (TTI).

\subsubsection{Source of Travel Time Unreliability}

The source of travel time unreliability can be related to supply and demand variations. On the supply side, the unreliability sources include incidents, work zones, weather, traffic control, management dynamic pricing, and variation in individual driving behaviors that affects network performance. On the demand side, special events, day-to-day variation in 
individual behaviors, unfamiliar users are considered as source of unreliability (Dong and Mahmassani 2009; Mai et al., 2012).

Table 2-2 Definitions and Use of Reliability Performance Measures (Hadi et al., 2014)

\begin{tabular}{|c|c|c|}
\hline $\begin{array}{c}\text { Reliability } \\
\text { Performance } \\
\text { Metric }\end{array}$ & Definition & $\begin{array}{c}\text { Project } \\
\text { Using } \\
\text { Measure }\end{array}$ \\
\hline Buffer Index & $\begin{array}{c}\text { Buffer Index The difference between the 95th } \\
\text { percentile travel time and the average travel time }\end{array}$ & L03, L08 \\
\hline 95th Percentile PTI & 95th percentile of the TTI distribution & L03, L08 \\
\hline 80th Percentile TTI & 80th percentile of the TTI distribution & L03, L08 \\
\hline Skew Statistics & $\begin{array}{c}\text { The ratio of 90th percentile travel time minus the } \\
\text { median travel time divided by the median travel time } \\
\text { minus the 10th travel time percentile }\end{array}$ & L03 \\
\hline Misery Index & $\begin{array}{c}\text { The average of the highest 5\% of travel times divided } \\
\text { by the free-flow travel time }\end{array}$ & L03 \\
\hline Standard Deviation & Usual statistical definition & L08 \\
\hline Kurtosis & Usual statistical definition & L08 \\
\hline
\end{tabular}

\subsubsection{Incorporating Reliability into Operations Modeling Tools}

The SHRP2, L04 project (2014) proposed a framework to incorporate reliability into operations modeling tools that includes three main components: (1) Scenario Manager; (2) simulation tools; (3) Vehicle Trajectory Processor. First, the Scenario Manager, which accounts for exogenous unreliability sources such as special events, day to day variations, closures of alternative roads, and visitor demands as demand side variability factors. The scenario manager also captures incidents, adverse weather, and work zones as supply side exogenous unreliability sources. Second, the Simulation Tools that model sources of demand unreliability endogenously for those sources that can be accounted for in the simulations, including: flow breakdown at recurrent bottlenecks, incidents, traffic control, dynamic pricing, and heterogeneity in microscopic deriver behaviors that can be controlled 
into microscopic traffic. Third, the Vehicle Trajectory Processor, which extracts reliability information from the simulation output such as travel time distribution, reliability performance indicators, and users-centric reliability measures. Mahmassani, Kim et al. (2014) used the AIMSUN and DYNASMART-P simulation platforms, as examples of microscopic and mesoscopic tools for reliability estimation. Scenario Manager and Trajectory Processor prototypes were developed and interfaced with the microscopic and mesoscopic model to allow the reliability assessment.

\subsubsection{Scenario Manager}

The Scenario Manager is a preprocessor of simulation input files which allows the incorporation of exogenous sources of travel time variation in model runs. The Scenario Manager is capable of building scenarios of mutually consistent combination of external events that impact travel time reliability. A built-in Monte Carlo stochastic sampling in the Scenario Manager generates hypothetical scenarios with combinations of external events for analytical purposes.

\subsection{Monte Carlo Approach}

Scenario Manager may use Monte Carlo simulation to prepare input scenarios for propagating uncertainties in selected scenario components into uncertainties in the generated scenarios $S i(i=1, \ldots, N)$, which can provide travel time distribution. As shown in Figure 2-1, the Scenario Manager uses Monte Carlo simulation to produce hundreds of input scenarios by sampling from the joint probability distribution of the scenario components. Each scenario is equally likely, which allows the Trajectory Processor to 
aggregate travel time distributions from a large number of simulation runs to obtain the most probable outcome of a set of reliability performance indicators for the certain time and space domains. Figure 2-1 shows a schematic diagram illustrating Monte Carlo approach.

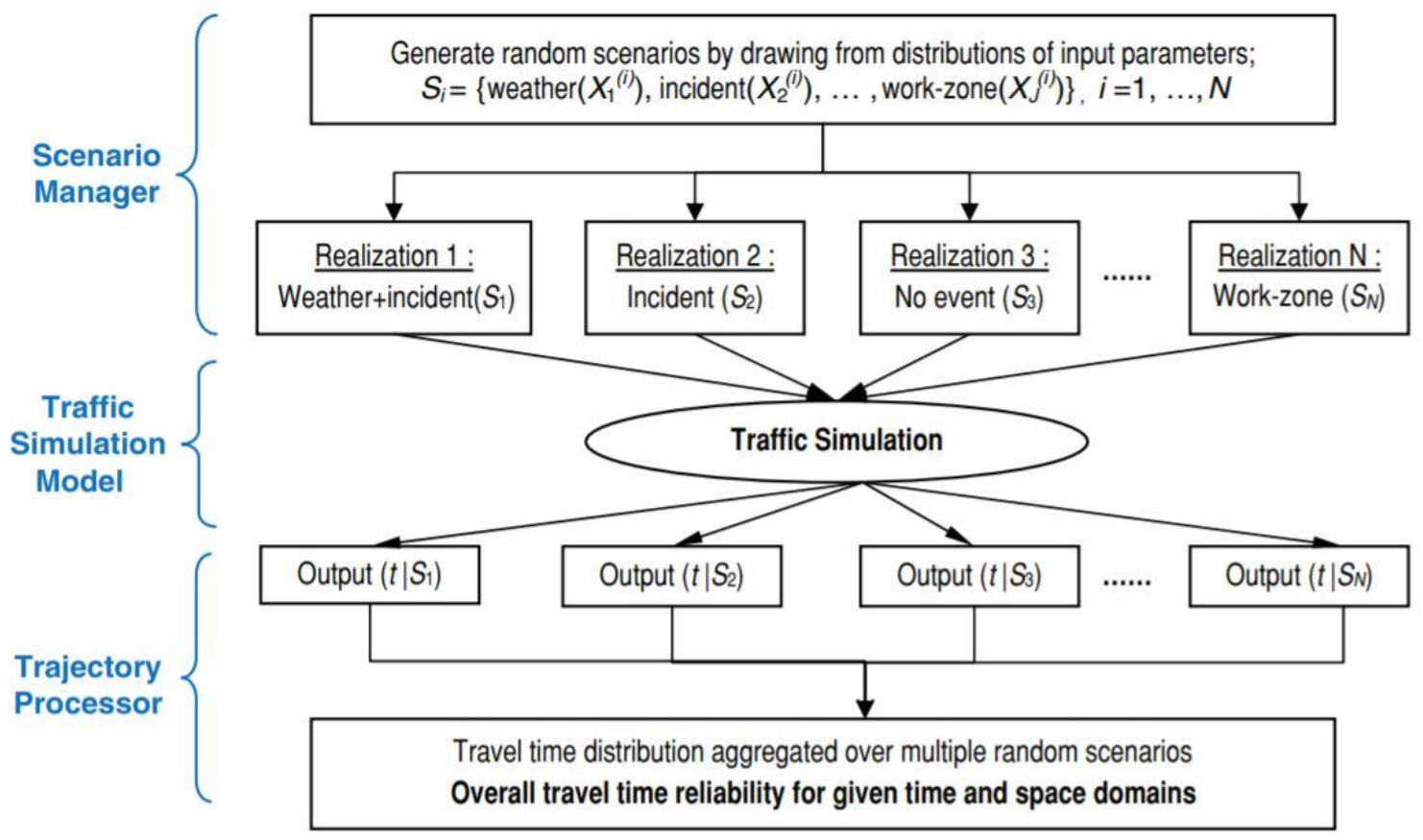

Figure 2-1 Monte Carlo Approach (Mahmassani et al., 2014)

\subsection{Mix-and-Match Approach}

The second approach that can be used with the scenario generator aims to generate input scenarios directly instead of generating the scenarios randomly. This is done by mixing and matching possible combinations of specific input factors or by directly utilizing known historical events or specific instances such as: holidays and football games. Figure 2-2 shows a schematic diagram illustrating this approach. 


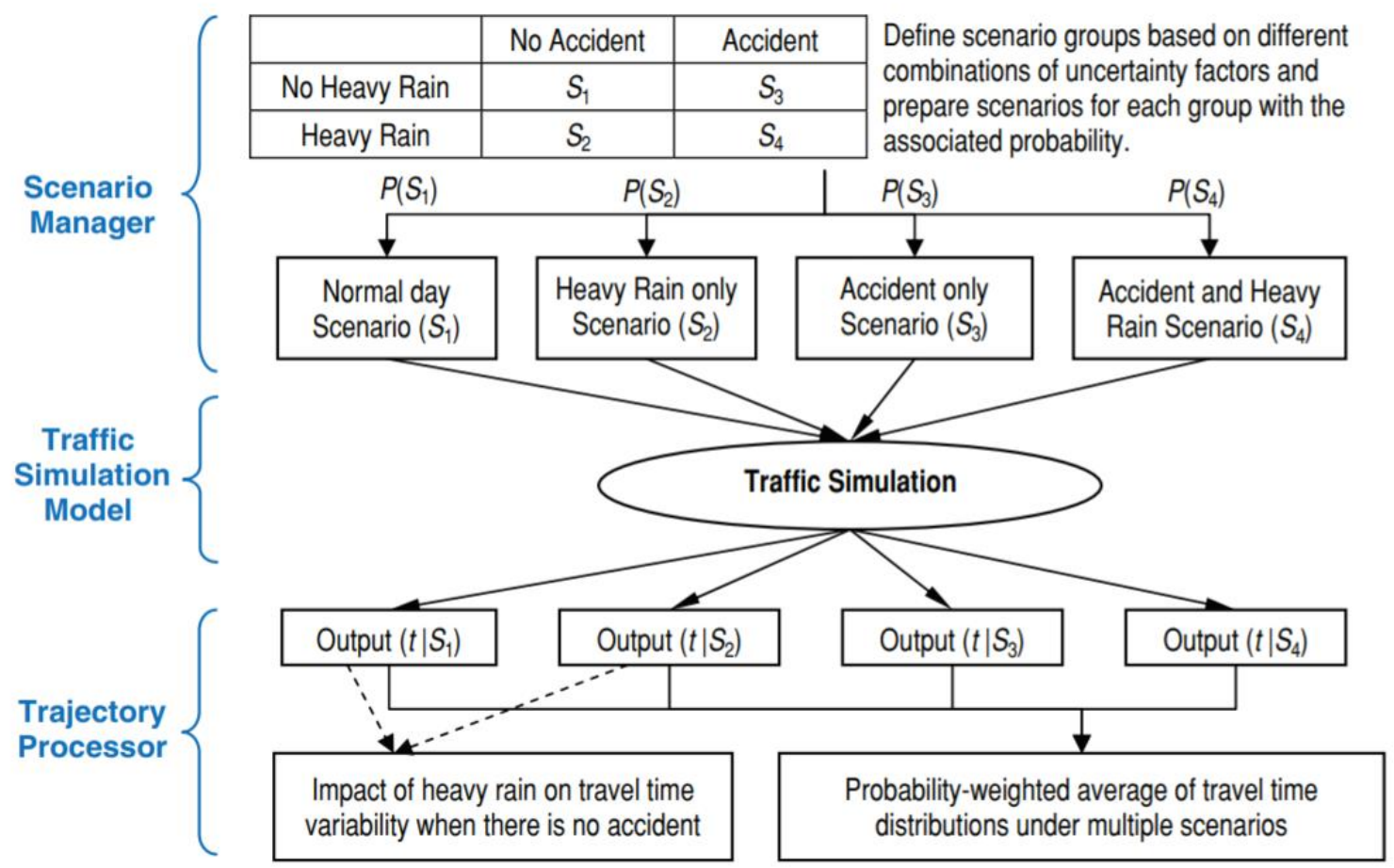

Figure 2-2 Mix and Match Approach (Mahmassani et al., 2014)

\subsection{Combined Approach}

This approach uses the Monte Carlo approach with a combined mix-and-match approach to generate a large number of scenarios for the entire scenario space and categorizing them into the associated groups to obtain the group probabilities.

\subsubsection{Vehicle Trajectory Processor}

The vehicle Trajectory Processor as implemented in the SHRP2 L04 project (2014) is designed to utilize traffic assignment simulation results for assessment of travel time reliability. The processor presents both O-D-level and path-level travel time statistics such as: average and standard deviation of travel time in table format which allows the identification of the pair's travel time. The most unreliable $\mathrm{O}-\mathrm{D}$ processor also produces 
O-D-level travel time distributions in graphical format including: Probability Distribution Function graph (PDF), Cumulative Distribution Function graph (CDF). Finally, the processor has the capability to display any possible paths between any O-D pair on Google Earth.

\subsection{Summary}

Previous studies show that ATM provides significant benefits in terms of travel time, travel time reliability, emission, fuel consumption, safety, and other performance measures of transportation systems. Although, the impact of ATM strategies on mobility measures have been widely investigated for freeway facilities, there is a lack of studies, that address ATM strategies for urban streets. In order to evaluate ATM strategies is necessary to use combinations of tools and software with different resolutions, and capabilities to take a full advantage of evaluation ATM strategies.

The evaluation of travel time reliability is critical to the assessment of ATM strategies. Improving reliability is one of the most important goals of implementing ATM strategies. The source of travel time unreliability can be related to supply and demand variations. On the supply side, the unreliability sources include incidents, work zones, weather, traffic control, management, dynamic pricing, and variation in individual driving behaviors that affects network performance. On the demand side, special events, day-today variation in individual behaviors, unfamiliar users are considered as source of unreliability. The SHRP2, L04 project (2014) proposed a framework to incorporate 
reliability into operations modeling tools that includes three main components: (1) Scenario Manager; (2) simulation tools; (3) Vehicle Trajectory Processor. 


\section{CHAPTER 3}

\section{METHODOLOGY AND OVERVIEW OF MODELING PROCESS}

\subsection{Introduction}

This chapter presents an overview of the methodology and tasks implemented throughout this study. The methodology follows sequential steps to achieve the goal and objectives of this research. The first step was the data acquisition. Data from multiple sources and agencies was collected and processed to develop and calibrate models. Next, a combination of tools were selected for the modeling and analysis of this research. After the tool selection, the next step was network preparation for modeling. The network geometry was imported, updated, and cleaned to better represent the existing real-world network. Different traffic patterns were identified for modeling to represent different demands and congestion levels. Then, the traffic network and demand parameters (supply and demand) of the simulation-based DTA models were calibrated for different demand levels utilizing real-world measures such as traffic flow, on each link and on each turning movement. The trip matrices were estimated for 15 minutes time intervals for input to the DTA models. An Origin Destination Matrix Estimation (ODME) process was implemented through three levels of calibration. In the first level (the network level), the overall network was calibrated. Since the arterial streets are the focus of this study, in the second level, a more detailed calibration of the demands on arterial street segments was conducted. In the third level, the demands of the intersections' turning movements were calibrated through a focus calibration on these movements. The next steps in the model preparation for modeling was 
to prepare the microscopic simulation model network. The calibrated model was imported from the simulation-based DTA tool to the microscopic tool. The combination of the two tools allowed the assessment of the traffic and incident responsive signal control.

This research also presents a methodology and framework to assess and design traffic and incident responsive signal control, as stated above. An important aspect of the developed methodology is determining the impacts of the interaction between segment incidents and adjacent intersections on signal operations. In addition, to supplement the DTA-based analysis, regression models were developed to estimate the diversion rate due to urban street incidents based on real-world data. These regression models were combined with the DTA model to estimate the volumes at the incident location, alternative routes, and cross streets considering diversion. These volumes were used as inputs to microscopic tool to better evaluate the impacts of the new signal timing plans on the network performance. The remaining subsections of this document discusses the methodology in more detail.

\subsection{Utilized Modeling Framework}

The multi-resolution modeling framework utilized in this study is based on the framework developed by Hadi and Xiao (2015) for modeling advanced strategies. Figure 3-1 illustrates this framework. As shown in this figure, the framework contains three components:

1. Data sources and tools that allow the utilization of data from multiple sources to support modeling tasks. 
2. Supporting tools that assist modelers in developing, calibrating, and processing the results of the selected modeling tools.

3. Modeling tools of different types and resolution levels that allow the estimation of various performance measures.

The details of these components, as applied to this research, are discussed in the following subsections.

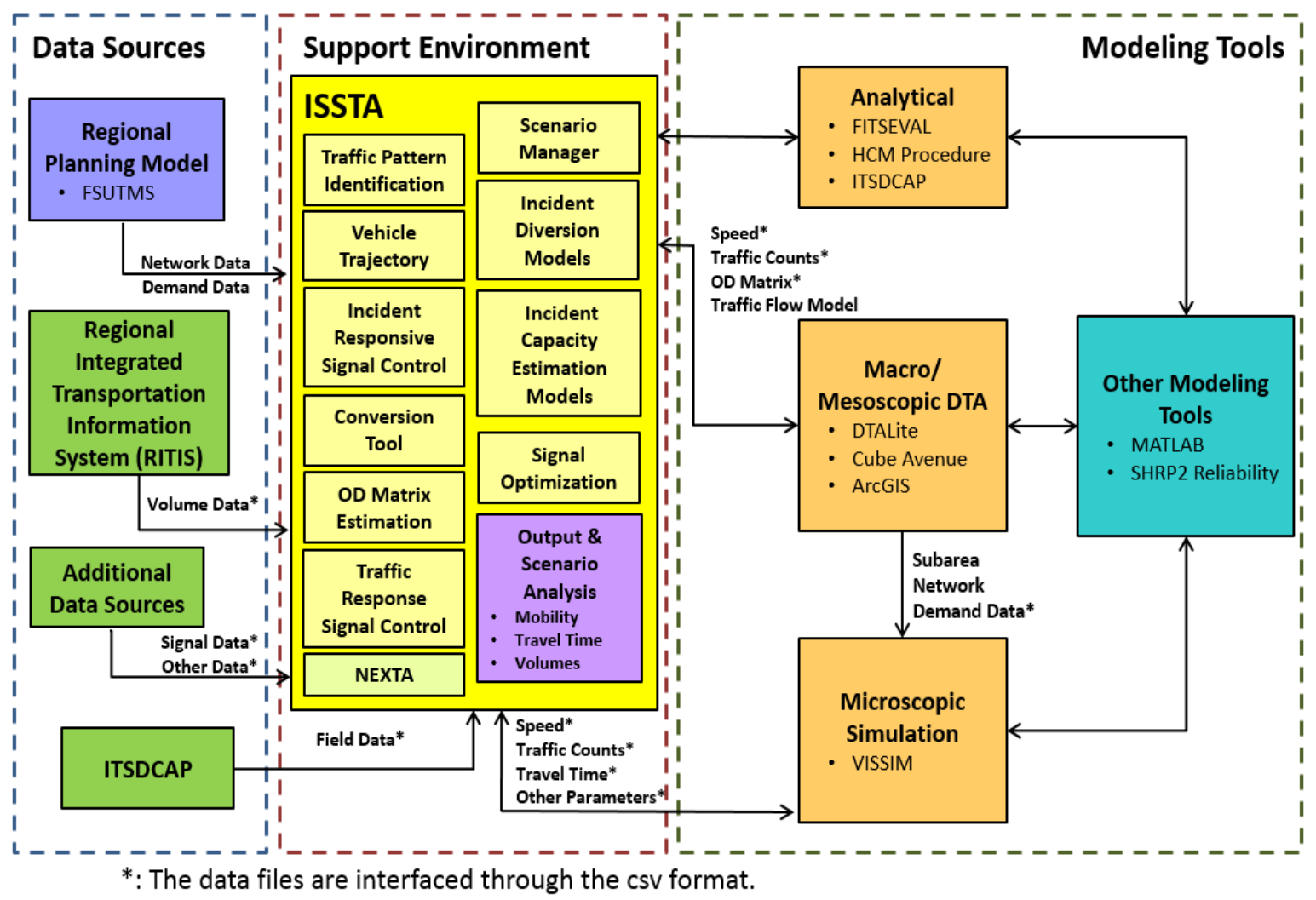

Figure 3-1 Utilized Modeling Framework

\subsubsection{Data Sources and Tools}

Advanced modeling requires high-quality processed data to ensure that the developed model applications accurately simulate existing and future real-world conditions 
under different scenarios and strategies. The needed inputs to these simulation models include roadway geometry and physical characteristics (for example, link length, free-flow speed, capacity, etc.), signal timings and control, travel times under different conditions, and dynamic origin-destination demand matrices or time-dependent vehicle trips. Modeling ATM strategies also require other parameters such as incident location and duration, work zone, weather, and special event statistics.

Travel demand forecasting models and archived real-world data are two main data sources that can provide the inputs for multi-resolution analysis. Archived real-world data was collected using ITS equipment and devices, third party vendors and agencies, conventional data collection methods, accident/incident reports, signal control agencies. The data from both source categories were processed and converted to a format accepted by the selected modeling tools.

For this study, the regional demand forecasting model provided data such as initial network geometry, origin-destination matrices, and attributes for each link, and so on. The utilized real-world data includes speed and volume counts including those provided by FDOT and those downloaded from the website of the Regional Integrated Transportation Information System (RITIS) (University of Maryland CATTI Lab, 2016): signal timing operational plans and their associated activation time, and incident data from FDOT. 


\subsubsection{Modeling Support Environment}

The supporting tools used in this study include:

- The Network Explorer for Traffic Analysis (NeXTA) tool developed by Zhou and Taylor (2014) was used to help converting mesoscopic model files into the format of the input files of the utilized microscopic simulation model.

- The ODME tool provided with the NeXTA tool was used for time-dependent O-D matrix estimation based on combinations of demand forecasting model outputs and real-world data.

- The Highway Capacity Software (HCS) was used for signal timing selection/optimization support.

- Additional modeling support methods were developed in this study to estimate capacity and diversion during incident conditions, as described later in this chapter.

\subsection{Network Preparation and Calibration for Multiresolution Analyses}

In this study, the DTAlite tool was selected as the mesoscopic simulation based DTA environment to model the subarea network and VISSIM was selected as the microscopic simulation tool for more detailed analysis. The subareas network used as a case study was cut and imported from the Cube software-based regional demand model. The network was imported to the NeXTA data hub for network and demand adjustments. The resulting model from DTAlite was exported to the HCS software for signal timing optimization and to VISSIM for more detailed modeling. The following steps describe in more detail the preparation of the network. 
- Step 1-Subarea network and demand matrix extraction: In this step, the selected subarea network in Broward County, FL was extracted from the southwest Florida Regional Planning Model (SERPM) demand forecasting model coded in Cube. The extracted network includes the geometry and the origin-destination demands of the network. However, both the demands and network were modified, as describing in Steps 3 and 4.

- Step 2-Importing the extracted network and the demand into NeXTA: In this step, the extracted network and demands were imported to NeXTA to allow further adjustment and conversion.

- Step 3-Network Modification: This step included modifying the imported network to correct coding errors and to add geometry details that were not included in the demand model. In addition, it included adding signal timing control parameters for the signalized intersections.

- Step 4-Demand Estimation: This step included further refinement of the OriginDestination demands using a ODME procedure that attempts to reduce the differences between the estimated volumes from the assignment values and the measured values, while also reducing the deviation from the extracted O-D matrices from the demand model. 


\subsection{Traffic and Incident Responsive Signal Control Modeling}

The multi-resolution model developed and calibrated, as described above was used to model traffic and incident responsive signal control. The methodologies used in this modeling are described in Chapters 5 and 6.

In order to design the incident responsive signal control, it is necessary to estimate the capacity impacts due to arterial incidents that result from the interaction between the drop in capacity below demands at midblock urban street segment locations and upstream and downstream signalized intersection operations. The models were developed in this study based on data generated utilizing a microscopic simulation model. These developed models were used to set the capacity during the incident conditions in the mesoscopic based DTA tool (DTAlite) to estimate the diversion during incidents. In addition, to supplement DTA-based analysis in estimating diversion, regression models were developed to estimate the diversion rate due to urban street incidents. These regression models were combined with the DTA model to estimate the volumes at the incident location, alternative routes and cross streets. This research also developed a method to estimate incident delay using a combination of the delay due to queuing on the incident link and the increase in upstream intersections delay due to the reduction in the saturation flow rate or maximum throughput resulting from queue spillback at the upstream intersection. The first component was calculated using deterministic queueing analysis equation, as is used in estimating incident delays on freeways. This method estimates the total delays based on incident duration, mean arrival rate (demand), and mean capacity under incident condition. The developed 
method can be used to estimate the impact of modifying signal timing plans during incidents with consideration of the estimating drop in capacity. 


\section{CHAPTER 4}

\section{DTA MODEL PREPARATION AND CALIBRATION}

\subsection{Introduction}

This chapter discusses the network preparation and calibration of the mesoscopic simulation-based DTA model utilized in this study (DTALite). In this study, the network and an initial estimation of the associated trips were extracted from a regional planning model. The case study network calibration, editing efforts and refinement of the initial demand for use in the DTA tool are discussed in this chapter.

\subsection{Network Preparation}

A subarea network around the Port of Everglades in Fort Lauderdale, Florida, was used as a case study for demonstrating the methodology of this study. As shown in Figure 4-1, this subarea covers the area between W. Sunrise Boulevard and Griffin Rd in the northsouth direction and between Florida A1A and SR 7 in the east-west direction. This subarea has a total number of 205 centroid zones and 2047 links. The original network was coded in the Cube Avenue software by Citilabs Inc. (Citilabs, Inc., 2016). The extracted subarea network had to be updated before use for analysis since the details and accuracy of the network in demand forecasting models are not sufficient for DTA applications. The network geometry were updated and cleaned to better represent the existing real-world network based on the GIS true shape. At the first step, the subarea network was converted 
into the link and node Shape files format from the Cube network format, allowing the links' curvature to be revised based on the real curvature using the GIS modification tool. All of the links' lengths were updated based on the identified curvatures.
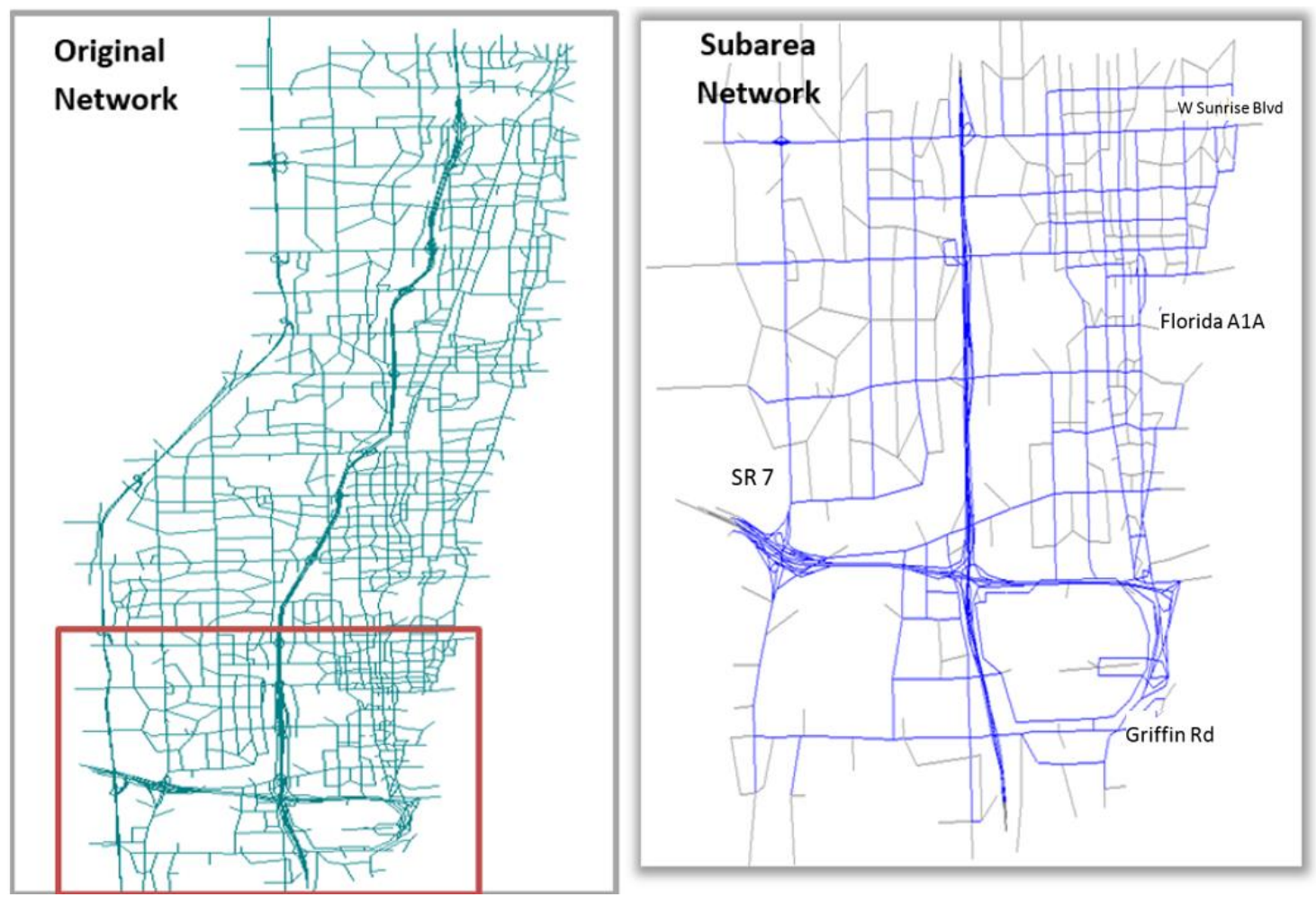

Figure 4-1 Original and Extracted Subarea Network

Link attributes, such as length, number of lanes, free-flow speed, capacity, facility type, and so on, were further checked and updated as necessary.

\subsection{Demand Data}

The 15-minute origin-destination (O-D) matrices during the PM peak period between 3:00 pm and 6:00 pm were extracted from a DTA network coded utilizing the 
Cube Avenue DTA tool developed by Citilabs in a previous study, utilizing the "subarea analysis" process. The Cube demand matrices were then converted into csv files and imported into DTAlite. Note that these demand matrices were obtained using the ODME procedure in Cube based on three-day Portable traffic monitoring sites (PTMS) traffic counts at midblocks. Since this study focuses on arterial ATM, the accuracy of the estimation of turning movement counts is critical. The ODME process normally utilized is based on segment demand measurements. This process may not produce accurate estimates of the turning movement counts. Therefore, an additional ODME process had to be conducted in this study based on turning movement counts and MVDS data to allow better estimates of turning movement demands, as documented later. The matrices produced using Cube Avenue in the previous study were used as initial seed matrices in the additional ODME process, described later.

\subsection{Network and Demand Data Conversion to DTALite}

In this study, the network was imported to the NeXTA/DTALite tool through the link and node GIS shapefiles that were exported from the Cube Avenue network mentioned earlier. The corresponding node and link attributes, such as the number of lanes, free-flow speed, lane capacity, link type, and so on, were configured in the "Configure GIS Importing Settings" file of NeXTA. The link types coded in the Cube Avenue model were also converted into the link types used in the DTALite, as shown in Figure 4-2. Thus, this study converted the cube link type to DTALite link type. It should be mentioned that the original Cube network consists of both one-way and two-way links. However, the NeXTA 
importing interface can only handle the network with either one-way or two-way links but not both. Therefore, the network was first imported as two-way links and the extra links were manually deleted for those one-way links. Figure 4-3 shows an example of these modification.

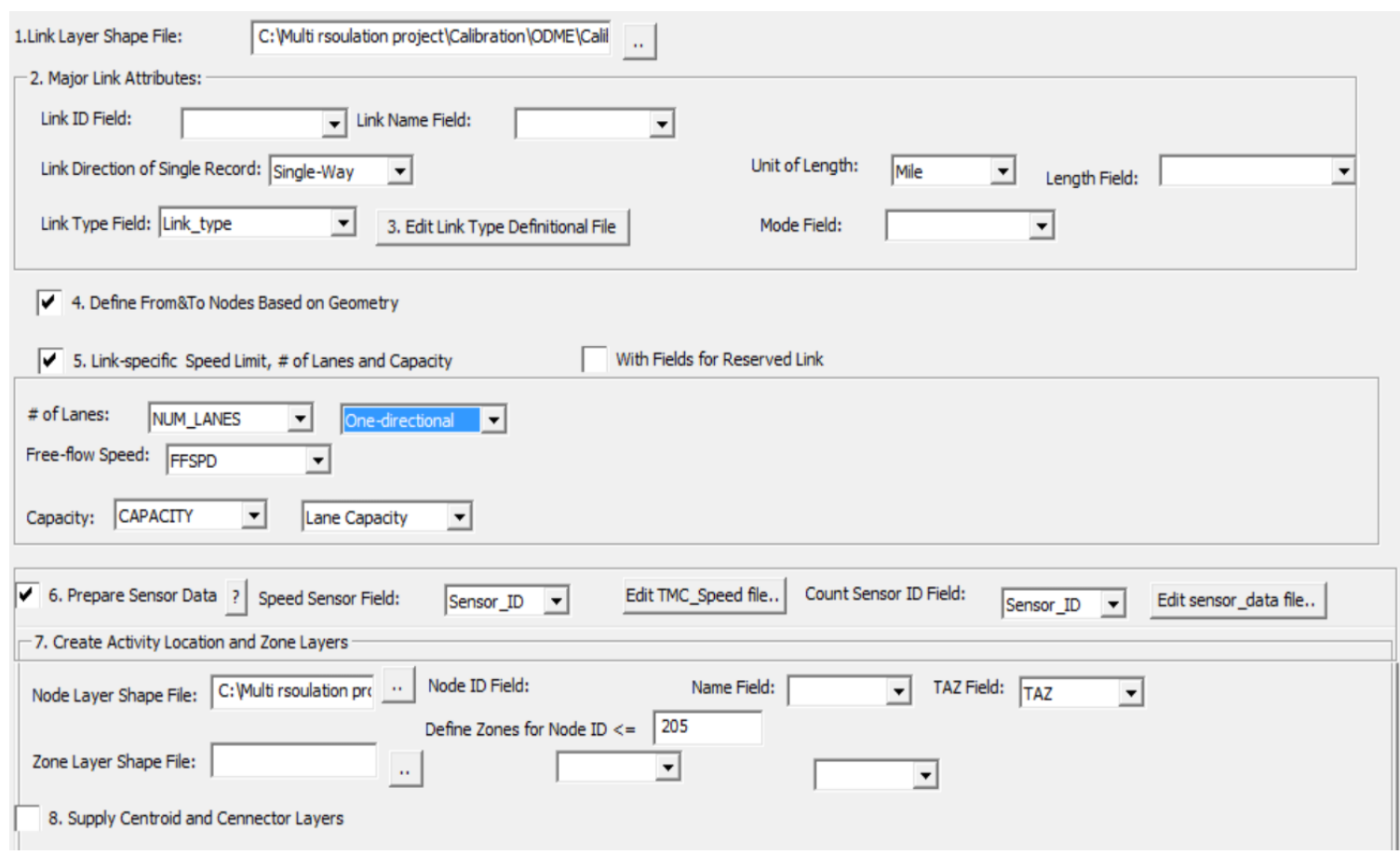

Figure4-2 GIS importing setting Interface in NeXTA

\subsection{Construction of signal and Junction Input Data}

Coding signal control correctly is critical for assessing the impacts of ATM strategies along arterials. Signalized intersection data for 30 main intersections, as shown in Figure 4-4 were coded in the network using the junction data file (*.IND). The junction data were obtained from the Cube Avenue model, which includes the signalized intersection geometry and signal timing data. Such information was coded in the DTALite model developed in this study through the "Node Movement Data" interface. Since the 
Broward Boulevard is the corridor that this study focuses on in the microscopic-level simulation, the signal timing data for this corridor were further updated based on the more detailed information coded in a VISSIM model obtained from FDOT District 4.

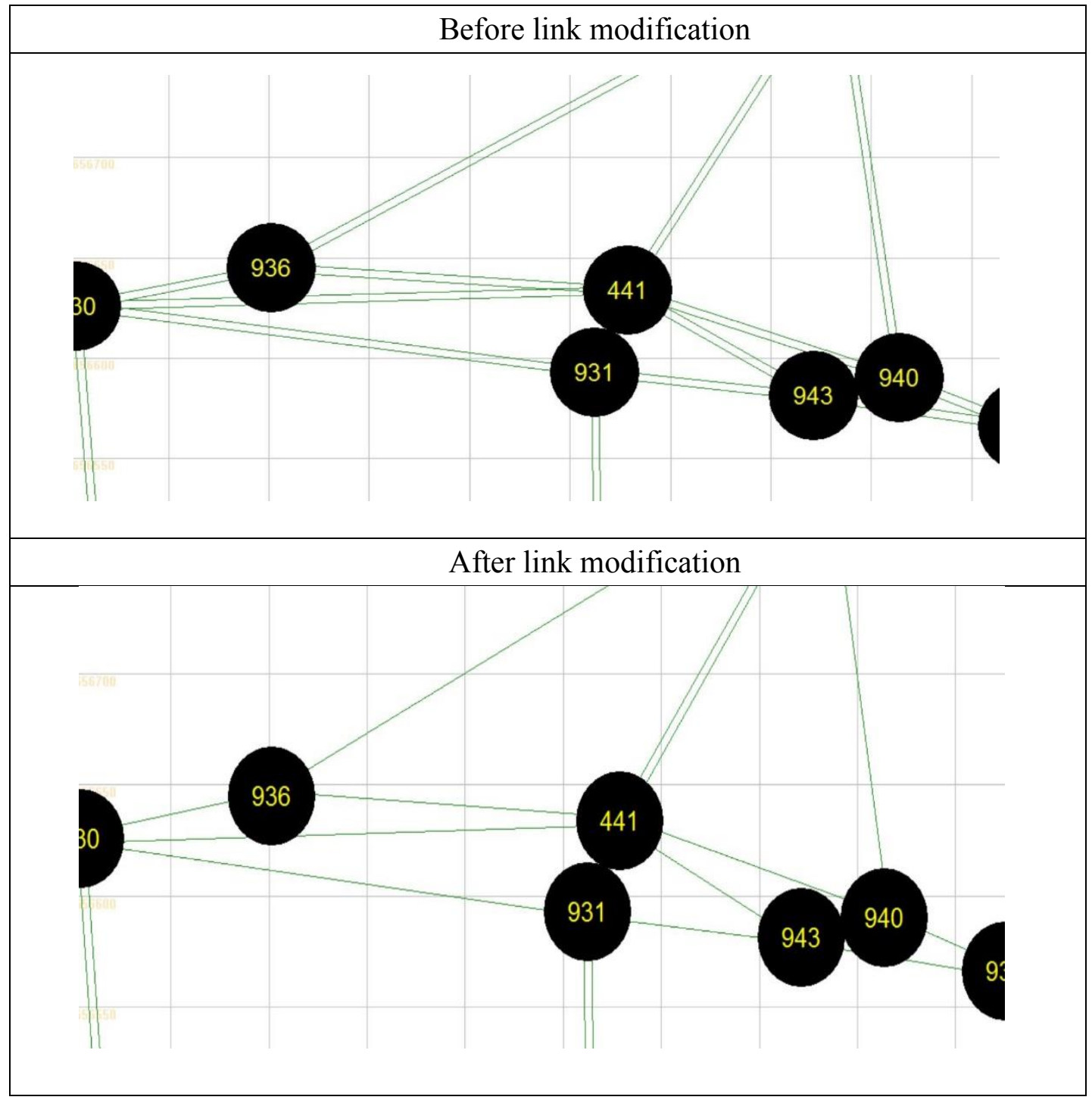

Figure 4-3 Example of Link Adjustment in NeXTA

As an example of the signalized intersections coded in DTAlite, Figure 4-4 shows the movements and signal timing for the intersection of Broward Boulevard at US 1. 


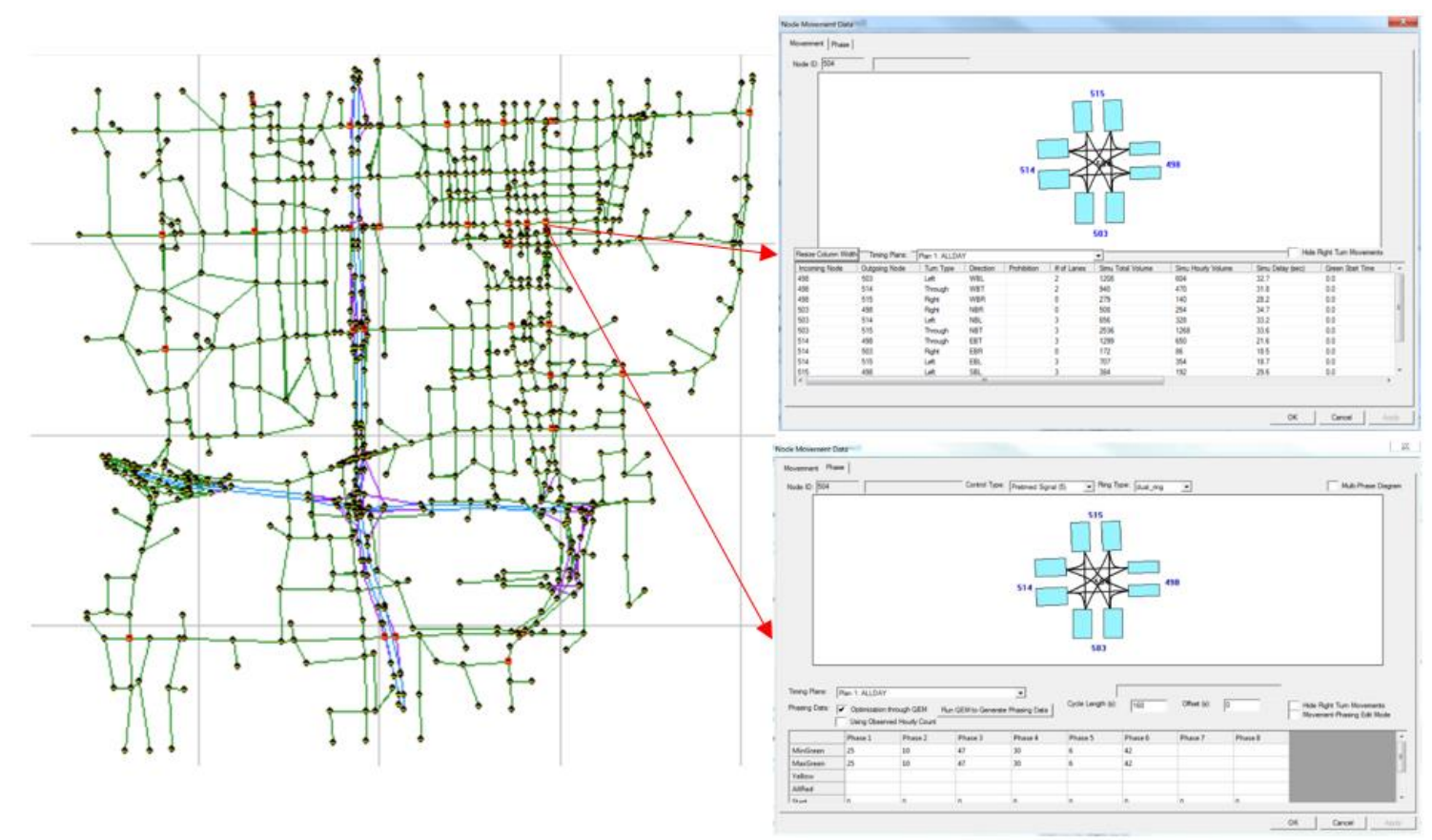

Figure4-4 "Node Movement Data" Interface for the Intersection at the Broward Boulevard and US-1 in NeXTA

\subsection{Data Acquisition and Traffic Pattern Identification}

In this study, data from multiple sources were collected for the analysis of ATM strategies, including point traffic detector data along freeways, Microwave Vehicle Detector System (MVDS) along major arterials, turning movement counts, event data such as incidents, and weather data. MVDS data was collected by the FDOT District 4 Transportation System Management and Operations (TSM\&O) program. This data was extensively used in this study for demand estimation, model calibration, and model validation. The data contains records of traffic volumes, speeds, occupancies, and travel times aggregated at 15-minute intervals. The point traffic detector data for the two freeways within the study area, I-95 and I-595, was obtained from the Regional Integrated Transportation Information System (RITIS) website. One-year data from 06/01/2015 to 
05/31/2016 between 3:00 pm and 7:00 pm was downloaded from this website. The locations of the detectors were identified and associated with links in the network that was modeled in DTAlite. Incident data were obtained from FDOT District 4 Transportation Management Center (TMC). This data include detailed event information, such as incident location, incident type, severity, duration, number of lane blockages, and so on. Archived turning movement counts were retrieved from the Broward County website. In addition, trips records that are within or passing through the study area in the month of January, 2016 were also extracted from a private sector data provider (INRIX) data to obtain the number of partial trips. The validity of all these data were checked. The erroneous data or the data violating the spatial or temporal consistency were eliminated.

Traffic patterns vary day by day. As described earlier in this document, the recent guidance and demonstration projects funded by the FHWA have emphasized the need to model days with different patterns (such as different demand levels, incident conditions, and weather conditions). This is particularly needed when modeling advanced strategies of the type addressed in this study.

This study acknowledges the need to model different traffic patterns and demonstrates the use of different patterns in the analysis. Representative days with different demand levels were selected for modeling. These days represent weekdays without incidents or abnormal external conditions such as heavy rain. In this study, the days with incident, special events, and bad weather were first filtered out, and also holidays 
and weekends were removed from the analysis. The remaining days were included in the next step of the analysis.

The 15-minute traffic counts between 3:00 pm and 7:00 pm for the remaining (event-free) days were aggregated to obtain the PM peak period traffic counts. Since this study focuses on arterial streets, the average volumes of four critical arterials (that is, the Broward Boulevard, Sunrise Boulevard, SR7, and A1A) were used to identify the network traffic patterns with different demand levels. Figure 4-5 shows the average volume per lane during the PM peak period for normal days between June 1, 2015 and May 31, 2016.

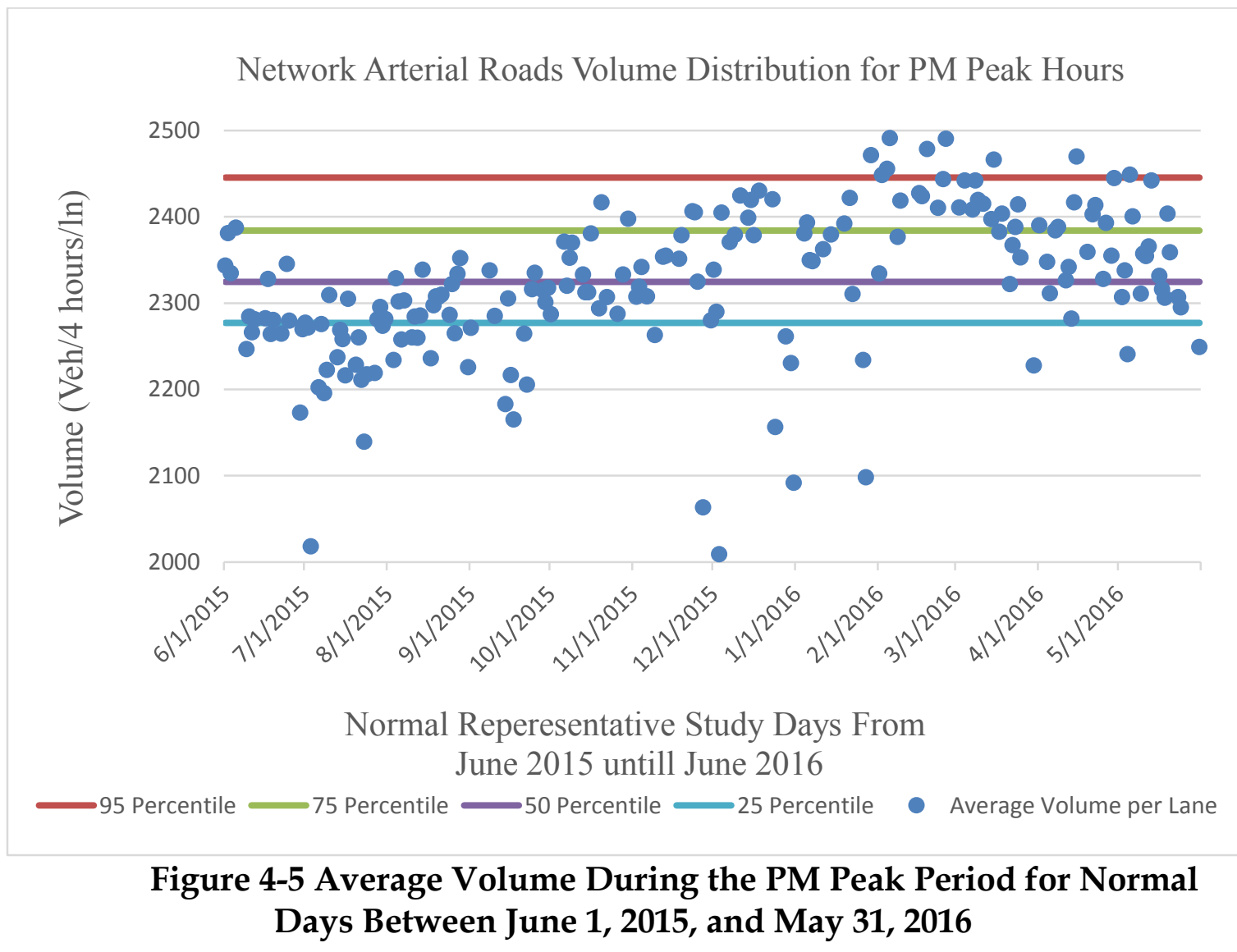


Previous ATM evaluations have been done for one day that is assumed to be representative of the operations for the whole year. However, the impacts of ATM strategies vary between days due to the variations in demand, weather, capacity, work zone, incidents, and other events. To better capture the variations in demand and quantify the benefits of ATM strategies, the $50^{\text {th }}, 75^{\text {th }}$, and $95^{\text {th }}$ percentile volumes of normal days during the PM peak period were identified, and shown in Figure 4-5 as straight lines. The days with volumes that are close to these three percentile values were considered as representative days for these four demand levels and used in later analysis. Note that there may be no days with exact volumes as those percentiles. In this case, the average volume of two closest days were used in the analysis. Figure 4-6 shows the variation of days with average volume for different percentiles.

\subsection{Supply Calibration}

The network or supply calibration process estimates and modifies the network parameters such as capacity and traffic flow model parameters. In this study, updates were made to the signal timing operation plans for signalized intersections along the study arterial street, as mentioned above. The saturation flow rates of approaching links to these signalized intersections were also updated based on the saturation flow rate estimated for the study area, that is, 1,800 passenger car per hour per lane for signalized intersections. 


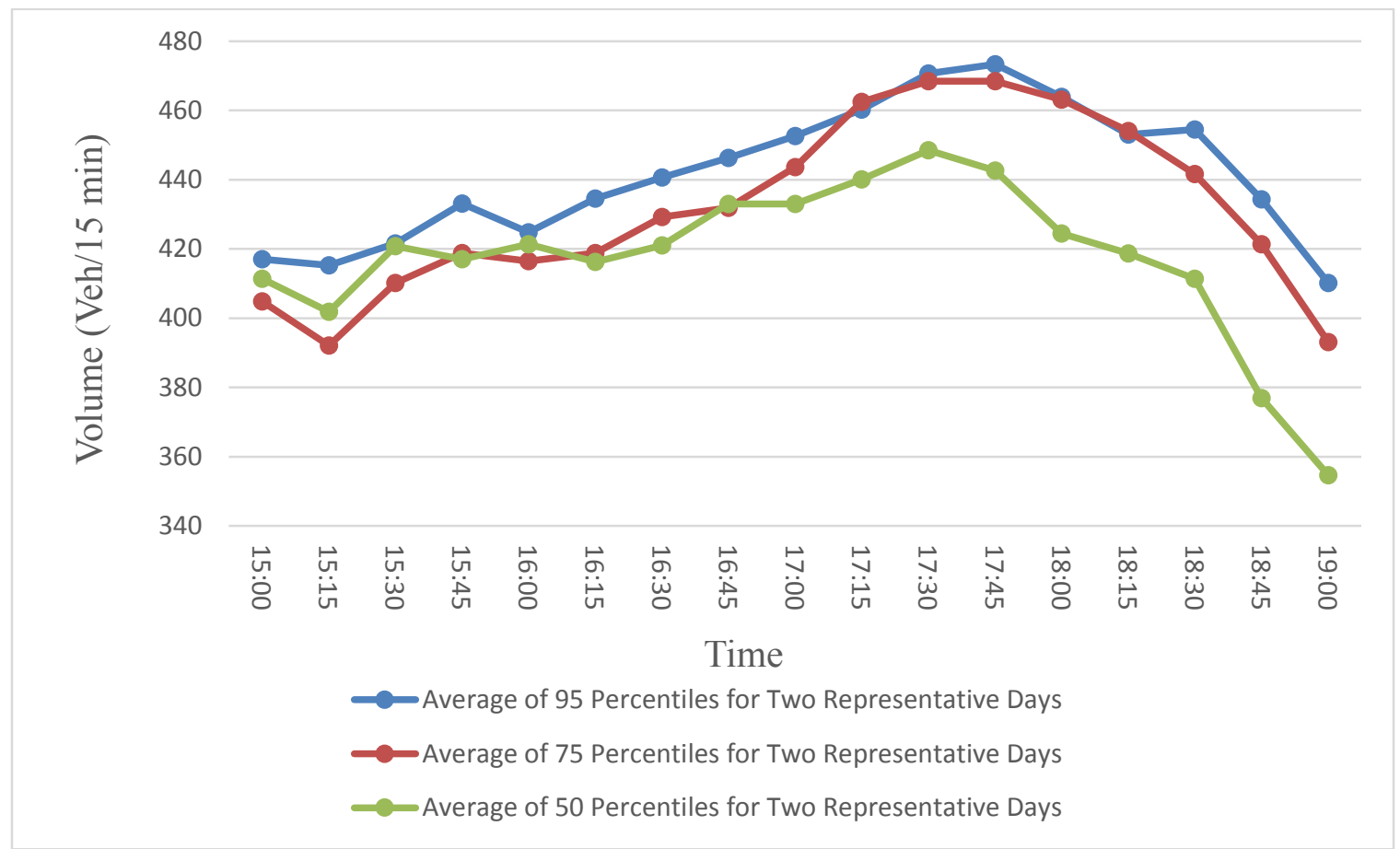

\section{Figure 4-6 Variation of Average Volume for Days with Different Percentiles}

Zone centroid connectors are usually coded with a very high capacity in regional demand models to avoid their impacts on traffic assignment. However, it was found in this study that such high capacities for zone centroid connectors cause unrealistic traffic assignment in DTALite. When there are zone centroids located between two parallel streets, vehicles may use zone centroid connectors as a bypass to avoid congested streets due to their high capacities and thus shorter travel times. In order to overcome this problem, the capacities and speed limits of these zone centroid connectors were adjusted to lower values to prevent vehicles from using these links as a bypass. In addition, in many cases the zone connectors from the demand model do not correspond to real-world 
connections of the zones to the network. In such cases, the centroid connectors have to be updated and segregated to get realistic results.

\subsection{Demand Calibration}

The ODME module included in DTALite was first conducted to calibrate the demand utilizing the available ITS detectors along the freeways and the midblock volume counts along the arterial streets obtained from the FDOT. The initial demand matrix for this process was obtained from a previous study conducted by Citilabs that utilized counts from the FDOT PTMS system as inputs to the ODME module implemented in Cube. Figures 4-7 to 4-9 compare the simulated and observed link volumes with the initial matrix without using dynamic ODME and with using the dynamic ODME process in DTALite for the three demand levels investigated in this study. It is seen from these figures that the dynamic ODME process implemented in DTALite with the additional input volumes can greatly reduce the difference between simulated and observed link volumes. The $\mathrm{R}^{2}$ value increases from 0.756 to 0.907 in the case of the $50^{\text {th }}$ percentile demand level, while the $\mathrm{R}^{2}$ value increases from 0.702 to 0.922 in the case of the $75^{\text {th }}$ percentile demand level. For the $95^{\text {th }}$ percentile demand level, the $\mathrm{R}^{2}$ value increases from 0.655 to 0.896 . 


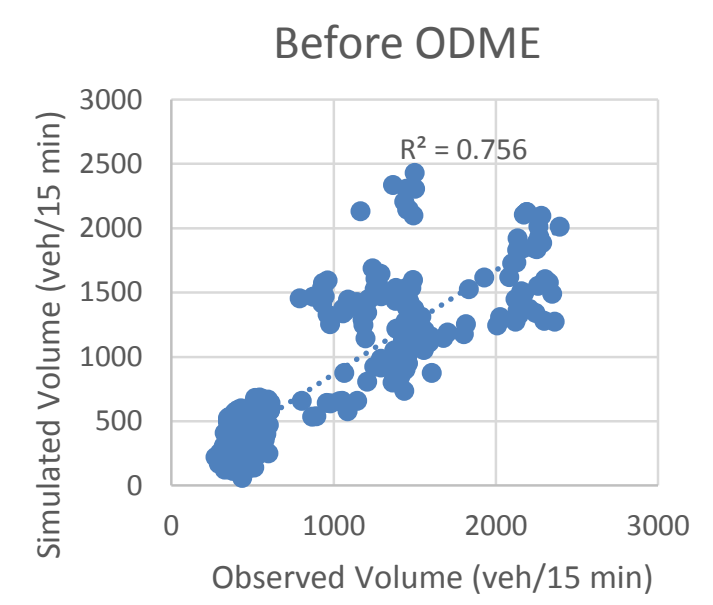

\section{Aftre ODME}

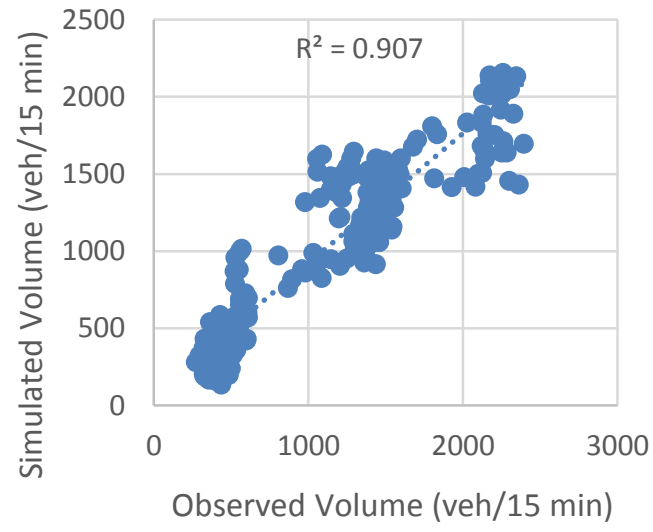

Figure 4-7 Simulated Link vs. Observed Link Volumes Before and After Running ODME for Day with $\mathbf{5 0}^{\text {th }}$ Percentile of Demand
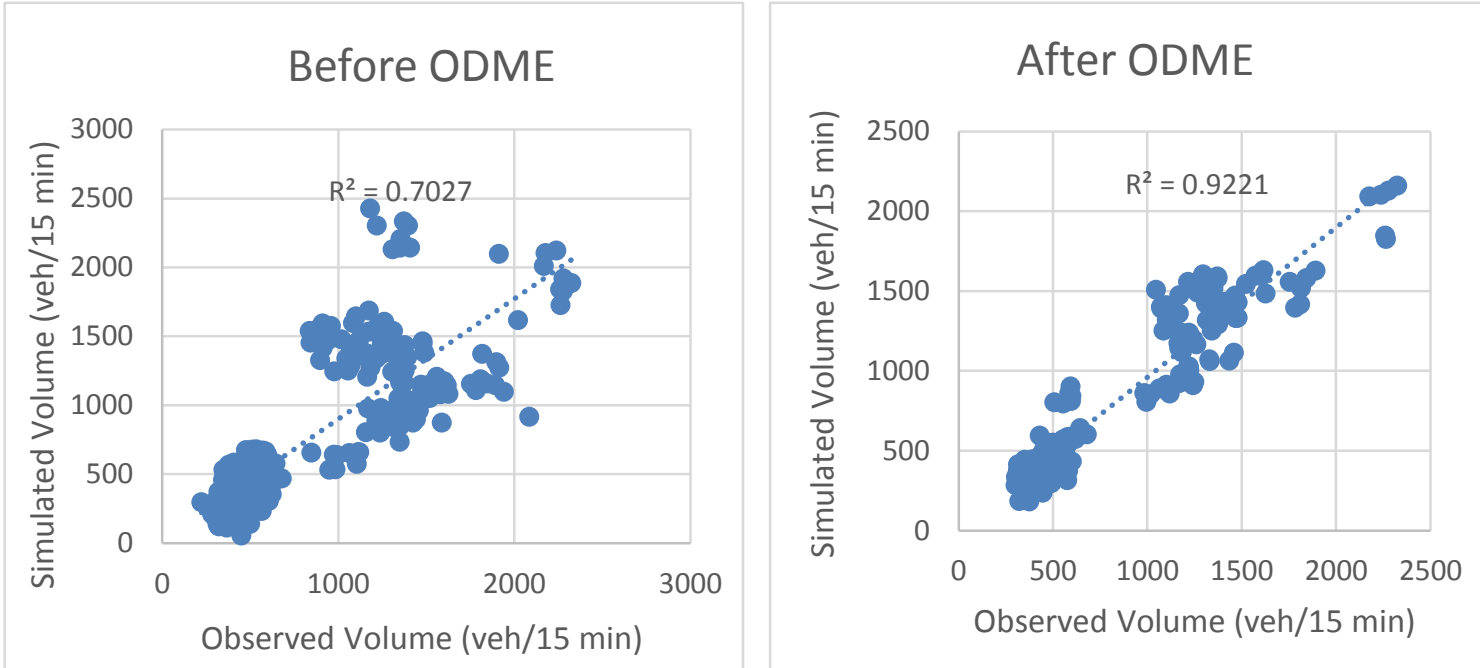

Figure 4-8 Simulated Link vs. Observed Link Volumes Before and After Running ODME for Day with $75^{\text {th }}$ Percentile of Demand

Even though the O-D matrix generated by the above ODME process produces very good $\mathrm{R}^{2}$ values for intersection approach volumes, there is significant errors at certain locations in terms of the turning movement percentages, which indicates that this model requires further calibration and validation. 

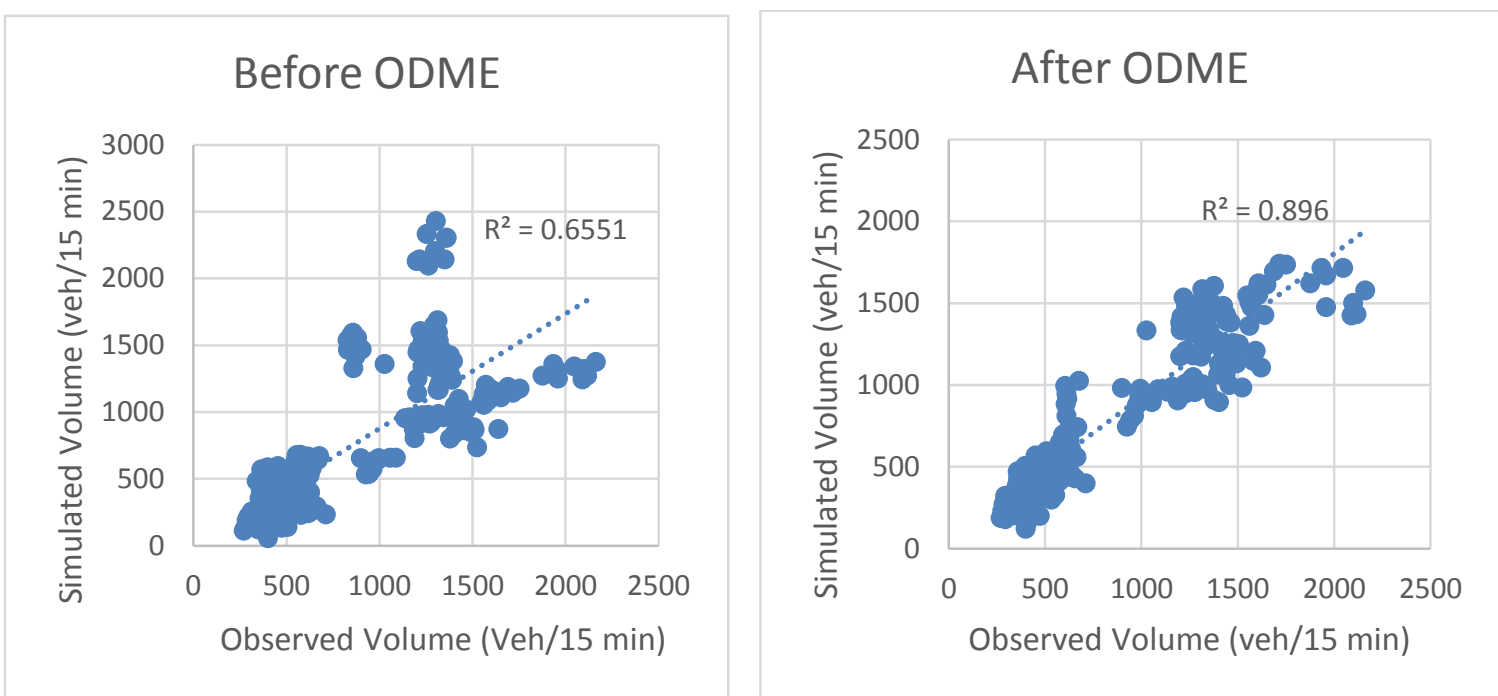

Figure 4-9 Simulated Link vs. Observed Link Volumes Before and After Running ODME for Day with $95^{\text {th }}$ Percentile of Demand

Therefore, an additional ODME process was conducted in this study for different percentile demand levels based on historical turning movement counts, in addition to the previously used midblock traffic counts, as described next and in the next chapter.

Since the DTALite ODME process does not allow the user to input turning movement counts, two approaches were tried. These two approaches are referred as the "virtual detector" approach discussed below and the "turning movement link" approach described in the next section. First, the virtual sensors were added to each approaching and departing links at the signalized intersections in this study with a purpose of minimizing the deviations from turning movement counts. Figure 4-10 shows the locations virtual sensors in the study network. Two signalized intersections are enlarged to show the detailed coding of MVDS and virtual sensors. The traffic counts at each virtual sensor were calculated by summing up all the movement volumes that can reach the corresponding 
link. However, the historical turning movement counts were only collected for one average day instead of one year. In order to produce the different turning movement counts for different demand levels, the ratios of MVDS counts at the $50^{\text {th }}, 75^{\text {th }}$, and $95^{\text {th }}$ percentile demand level to their average values were estimated and applied to the historical turning movement volumes in their vicinity to generate the turning movements at each demand level. Once the required turning movement counts were produced, the dynamic traffic assignment-based ODME procedure in DTALite was conducted again. It was noticed that the estimated O-D matrix still cannot produce reasonably good link volumes at certain locations compared to sensor data. A manual adjustment of the initial O-D matrix was made before running another ODME process. This process was conducted iteratively until reasonably good results were obtained. The adjustment described above considered the fact that the zone connections to the network derived from the demand model do not reflect the real-world conditions. In the future, it is recommended that a method is developed to automatically disaggregate the zones into subzones with realistic connections to the network. 


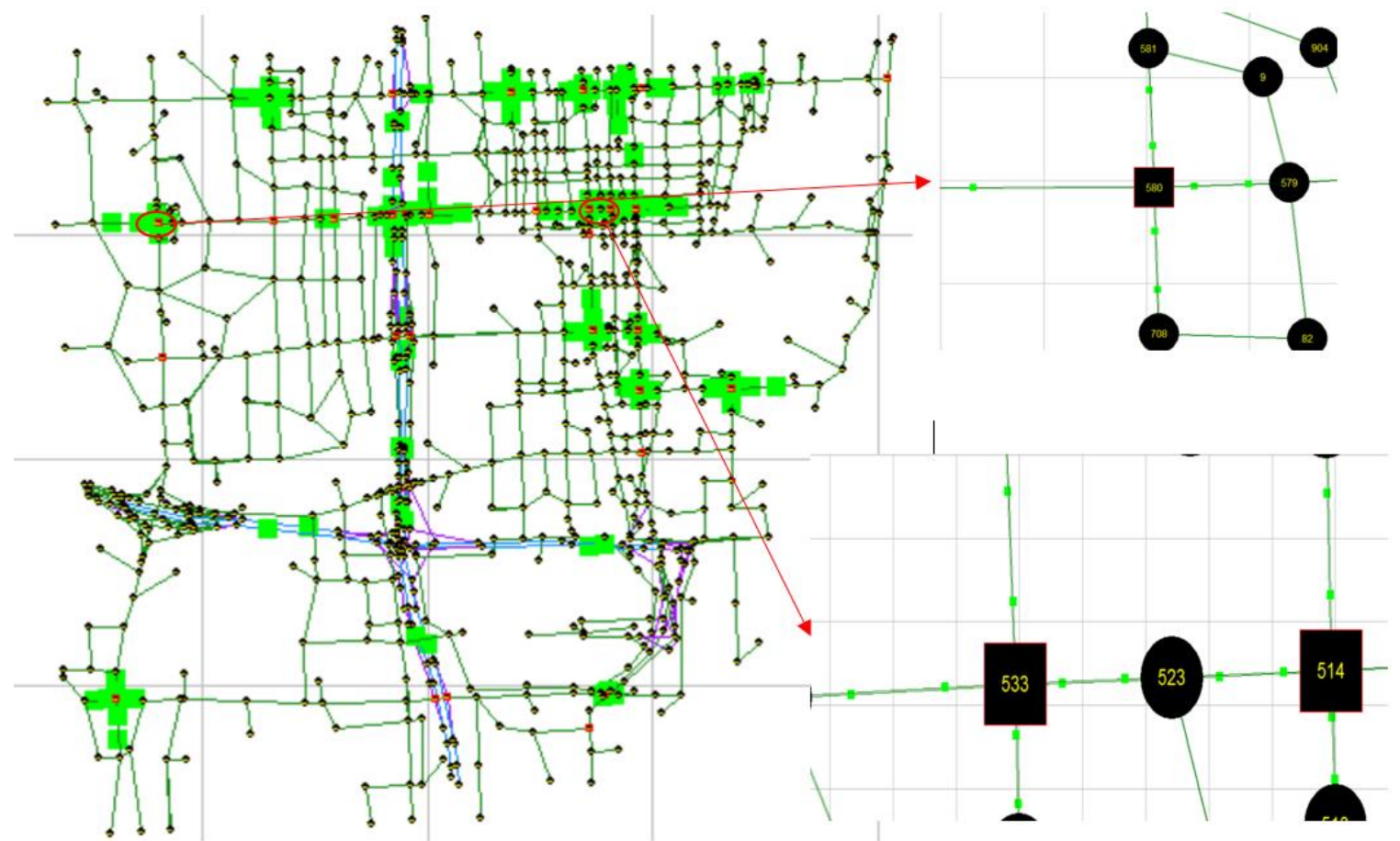

Figure 4-10 Virtual Sensor Locations in the Study Network

Figures 4-11 to 4-13 compare the simulated and observed turning movement counts along the Broward Boulevard before and after implementing the above ODME process for different demand levels. 

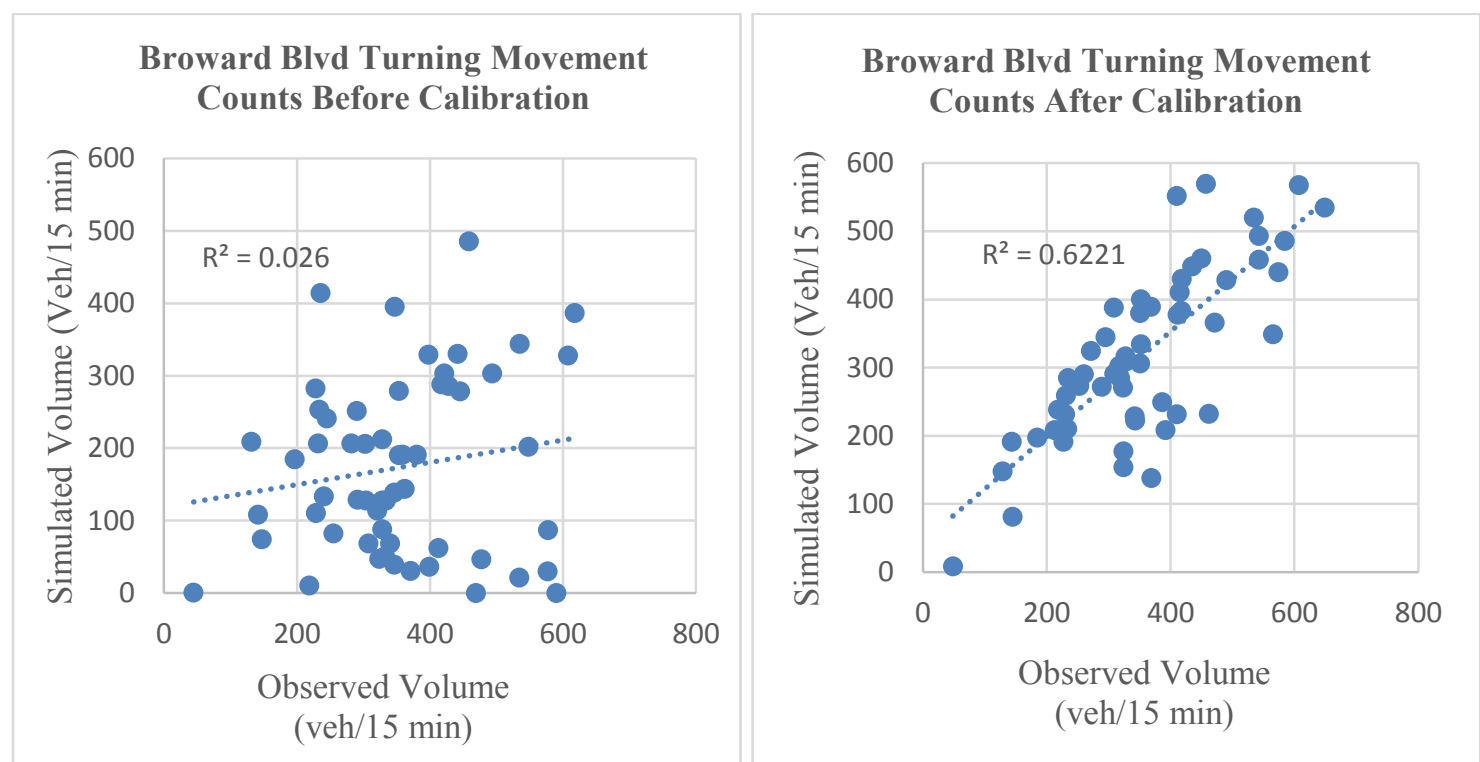

Figure 4-11 Simulated vs. Observed Link Volumes, Before and After Running ODME for the Day with $50^{\text {th }}$ Percentile of Demand
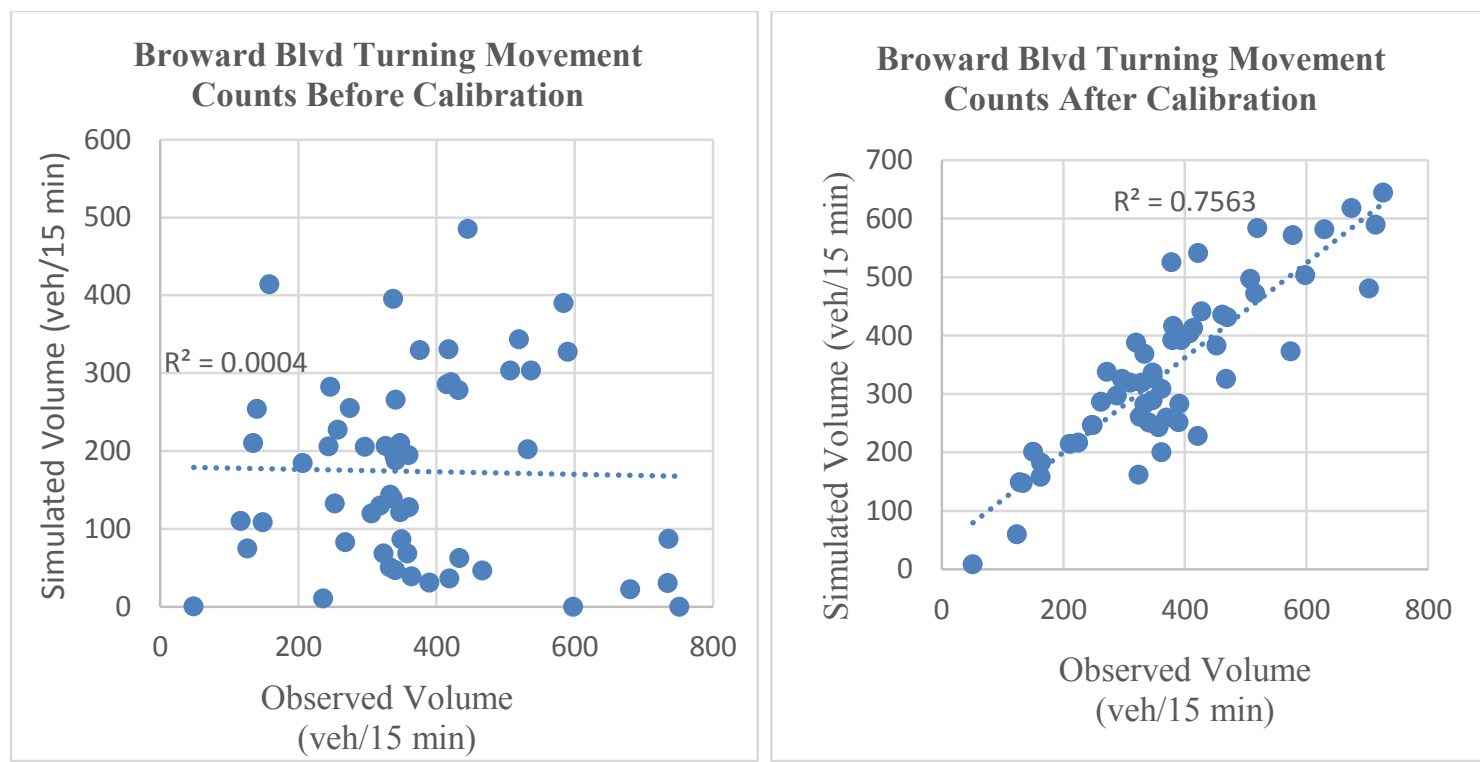

Figure 4-12 Simulated Link vs. Observed Link Volumes, Before and After Running ODME for the Day with $75^{\text {th }}$ Percentile of Demand

As shown in Figure 4-11, the $\mathrm{R}^{2}$ value was 0.026 without demand calibration for the median day. This $\mathrm{R}^{2}$ value can be improved to 0.622 after the ODME process, which 
produces turning movement volumes that are relatively close to the observed counts. Similarly, the results in Figures 4-12 and 4-13 show that the $\mathrm{R}^{2}$ value can be greatly increased from 0.0004 to 0.7563 for the $75^{\text {th }}$ percentile of demand and from 0.0008 to 0.7636 for the $95^{\text {th }}$ percentile of demand.
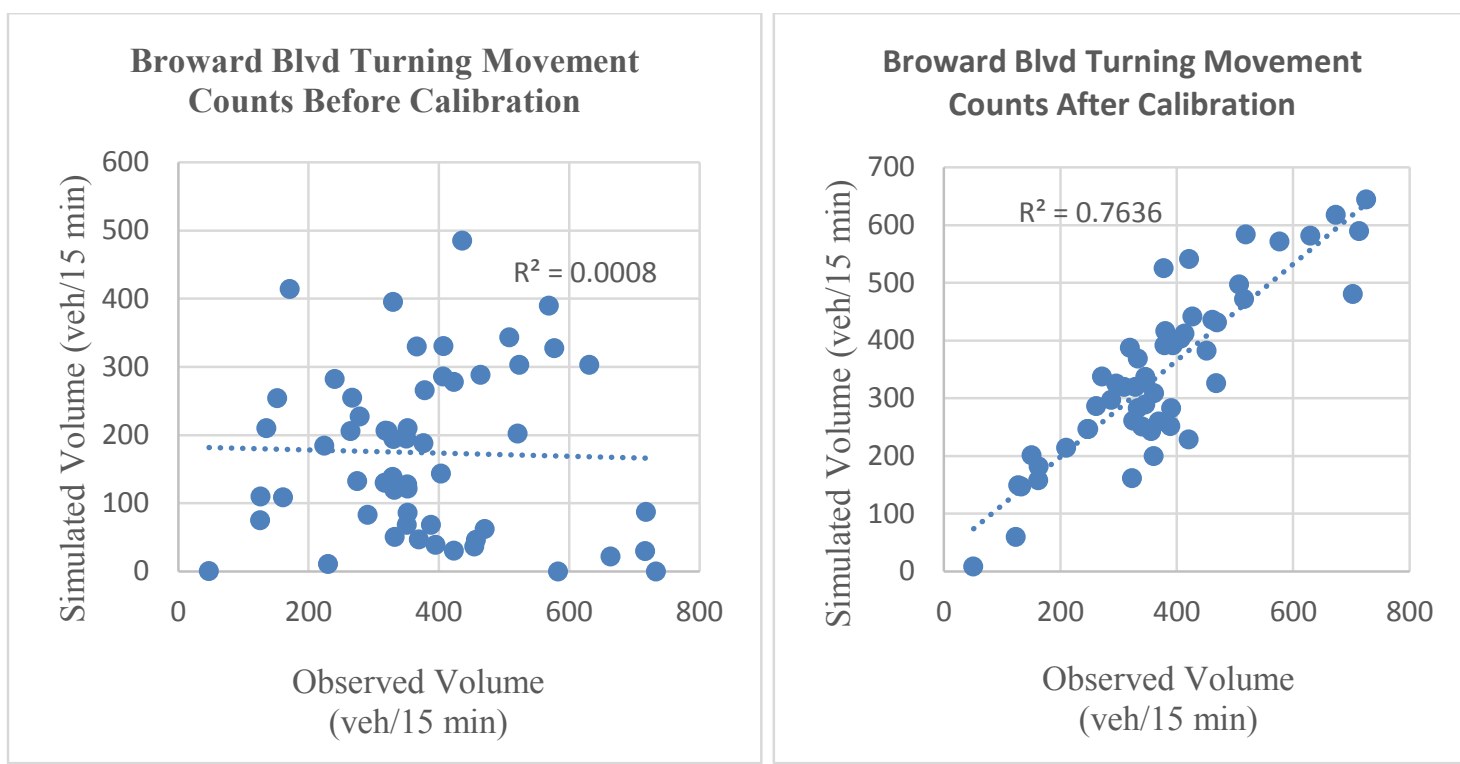

\section{Figure 4-13 Simulated Link vs. Observed Link Volumes, Before and After Running ODME for the Day with $95^{\text {th }}$ Percentile of Demand}

The accuracy of the calibration process was evaluated by comparing the estimated traffic turning movements counts with the observed ones. The Root Mean Square Error (RMSE) and Percentage Root Mean Square Error (PRMSE) were used as measures to assess the link simulated volumes in comparison to observed link volumes. The following equations provide the expressions for RMSE and PRMSE:

$$
R M S E=\sqrt{\frac{\hat{\mathrm{i}}_{\left(y_{i}-\hat{y_{l}}\right)^{2}}^{n}}{n}}
$$




$$
\text { PRMSE }=\sqrt{\frac{\sum_{\mathrm{i}\left(y_{i}-\widehat{y_{i}}\right)^{2}}}{n}} \times \frac{100 n}{\sum \widehat{y_{i}}}
$$

where

$$
\begin{aligned}
& y=\text { simulated/estimated volume, } \\
& \hat{y}=\text { observed volume, and } \\
& n=\text { number of predictions. }
\end{aligned}
$$

The RMSE and PRMSE measures between the simulated and real-world volumes were calculated. The corresponding results are listed in Table 4-1. As shown in this table, the RMSE is about 242 to 282 vehicles per 15 minutes and the PRMSE is $68 \%$ to $75 \%$ before the ODME process, while these values greatly drop to 79 to 89 vehicles per 15 minutes for the RMSE and $21 \%$ to $25 \%$ for PRMSE after the calibration process.

Table 4-1 Simulated Turning Movement Counts vs. Observed Turning Movement Counts for Broward Boulevard for Different Levels of Demand

\begin{tabular}{|c|c|c|c|c|}
\hline Demand Level & $\begin{array}{c}\text { RMSE Before } \\
\text { Calibration }\end{array}$ & $\begin{array}{c}\text { PRMSE } \\
\text { Before } \\
\text { Calibration }\end{array}$ & $\begin{array}{c}\text { RMSE After } \\
\text { Calibration }\end{array}$ & $\begin{array}{c}\text { PRMSE After } \\
\text { Calibration }\end{array}$ \\
\hline 50 percentile & 241.85 & $68.07 \%$ & 89.42 & $25.15 \%$ \\
\hline 75 percentile & 277.86 & $75.60 \%$ & 82.84 & $22.04 \%$ \\
\hline 95 percentile & 282.43 & $75.01 \%$ & 79.52 & $21.44 \%$ \\
\hline
\end{tabular}

It is also useful to look at the errors based on the count ranges. Table 4-2 shows the PRMSE values for three different ranges of observed counts. These values demonstrate that the simulated counts match better the observed counts after the ODME and calibration steps, especially for the observed count range of more than 500 vehicles per 15 minutes. It is seen from this table that the errors follow almost the same pattern for different demand 
levels. Compared to the other two demand levels, the $95^{\text {th }}$ percentile demand level has the least PRSME. The $75^{\text {th }}$ percentile demand level has a lower error in the count range of 250 to 500 vehicles per 15 minutes. Further, analysis to improve the accuracy of turning movement counts was conducted utilizing the turning vehicle link method, as described in the next chapter.

Table 4-2 Simulated Turning Movement Counts vs. Observed Turning Movement Counts for Broward Boulevard for Three Observed Volume Classes

\begin{tabular}{|c|c|c|c|c|c|c|}
\hline \multirow{2}{*}{$\begin{array}{c}\text { Count } \\
\text { Volume } \\
\text { Range }\end{array}$} & \multicolumn{2}{|c|}{$\begin{array}{c}\text { 50 Percentile } \\
\text { (Medium Day) }\end{array}$} & \multicolumn{2}{c|}{$\begin{array}{c}\text { 75 Percentile } \\
\text { (Heavy Day) }\end{array}$} & \multicolumn{2}{c|}{$\begin{array}{c}\text { 95 Percentile } \\
\text { (Very Heavy Day) }\end{array}$} \\
\cline { 2 - 7 } & $\begin{array}{c}\text { PRSE } \\
\text { Before }\end{array}$ & $\begin{array}{c}\text { PRSME } \\
\text { After } \\
\text { Calibration }\end{array}$ & $\begin{array}{c}\text { PRSME } \\
\text { Cefore } \\
\text { Calibration }\end{array}$ & $\begin{array}{c}\text { PRSME } \\
\text { After } \\
\text { Calibration }\end{array}$ & $\begin{array}{c}\text { PRSME } \\
\text { Before } \\
\text { Calibration }\end{array}$ & $\begin{array}{c}\text { PRSME } \\
\text { After } \\
\text { Calibration }\end{array}$ \\
\hline $0 \sim 250$ & $49.32 \%$ & $18.33 \%$ & $70.08 \%$ & $19.42 \%$ & $71.94 \%$ & $19.08 \%$ \\
\hline $250 \sim 500$ & $60.86 \%$ & $26.53 \%$ & $60.73 \%$ & $22.91 \%$ & $60.85 \%$ & $23.03 \%$ \\
\hline$>500$ & $73.95 \%$ & $19.33 \%$ & $79.23 \%$ & $17.90 \%$ & $78.94 \%$ & $15.53 \%$ \\
\hline
\end{tabular}

\subsection{Summary}

THIS STUDY COLLECTED DATA FROM DIFFERENT SOURCES

AND PROCESSED THEM TO MODEL AND CALIBRATE MODELS. CASE STUDY NETWORK WAS PREPARED FOR MODELING IN DTALITE. DIFFERENT TRAFFIC PATTERNS WERE IDENTIFIED FOR MODELING TO REPRESENT DIFFERENT DEMANDS AND CONGESTION LEVELS. THE DAYS THAT CORRESPOND TO DIFFERENT PERCENTILES OF TRAFFIC, SUCH AS THE 50TH, 75TH, AND 95TH PERCENTILES WERE DETERMINED AND MODELED USING A MESOSCOPIC-SIMULATION-BASED DTA. A SYSTEMATIC, MULTILEVEL APPROACH TO NETWORK CALIBRATION IS RECOMMENDED IN THIS STUDY, WITH AN INCREASING CALIBRATION 
SCOPE IN EACH LEVEL. ORIGIN-DESTINATION DEMANDS FOR TWO LEVELS OF CALIBRATION INCLUDING NETWORK LEVEL AND SEGMENT LEVEL FOR ARTERIAL STREETS WERE DETERMINED UTILIZING AN ODME PROCESS BASED ON A SEED MATRIX AND TRAFFIC COUNTS FOR THE THREE DAYS.

\section{CHAPTER 5 \\ TRAFFIC RESPONSE SIGNAL CONTROL}

\subsection{Introduction}

As stated earlier, this study focuses on utilizing a multi-resolution, multi-scenario approach to modeling dynamic signal control, as an example of modeling ATM strategies. This chapter discusses the modeling of demand responsive control. Chapter VI discusses incident responsive signal control. A combination of a mesoscopic simulation based DTA tool (DTALite), microscopic simulation (VISSIM), and a signal timing optimization tool is used to derive and evaluate demand responsive signal plans. This chapter discusses the methodology and procedure utilized for this purpose.

As discussed in the previous chapter, the DTALite models of the case study network with different traffic patterns were calibrated at the segment level, by comparing the results from the model with segment measures. An analysis of the results indicate that this calibration was not adequate to produce good turning movement volume estimates from the model runs. In this chapter, a portion of Broward Boulevard between US-1 and Andrew Boulevard, which is part of the network discussed in Chapter IV, was selected and 
further calibrated at the turning movement level. Once the DTAlite produced acceptable turning movement counts, the network was extracted from the mesoscopic DTALite network and input into the signal timing optimization tool and microscopic simulation tool to assess demand responsive signal control, as described below.

\subsection{Calibration of Turning Movement Volumes}

In order to design the traffic response signal control, it is necessary to calibrate the model further to ensure acceptable turning movement counts. The ODME procedure utilized as described in Chapter IV does not reach this level of calibration. The production of good turning movement counts from DTA models is particularly difficult and time consuming from dynamic traffic assignment models. The approach described in Chapter IV attempted to do this by summing the volumes of the turning movements leaving a link or those entering a link to produce additional "virtual detectors" that can improve the calibration accuracy. However, this approach still was not able to produce acceptable turning movement counts that can be used for designing traffic signal plans. Inputting the turning movement counts to the ODME process and coding the signal control utilized in the real-world are expected to help in producing better turning movement counts. Therefore, additional calibration of the turning movement counts was conducted for the case study in this chapter. Each turning movement was coded as a link as shown in the Figure 5-1 and the turning movement volume was associated with the link. This allows a better consideration of the turning movements in the ODME process. In addition, the 
research uses more refined zone connectors to ensure accurate representation of O-D trips and appropriate access to the network in the mesoscopic model. Since the locations of the zone connectors are important, their locations were matched with real-world network configuration to ensure that they provide reasonable access to the network that is similar to what is expected in real-life.

Before Adding Turning Movement Count Sensors




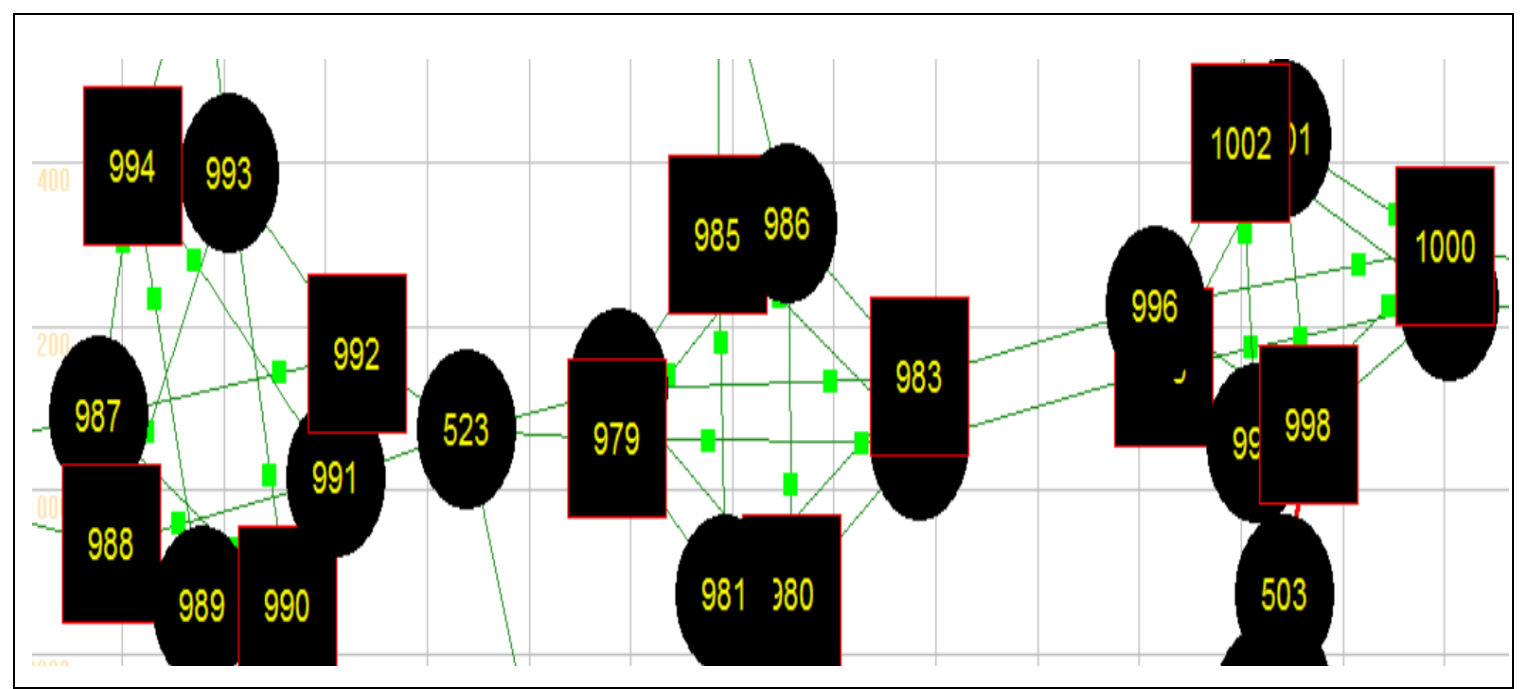

Figure 5-1 Turning Movement Sensor Adjustment in NeXTA

As illustrated in Figure 5-1, the turning movement counts were added to the three intersections within the mentioned case study with the purpose of minimizing the deviations from turning movement counts. The ODME process was conducted to calibrate the demand utilizing the observed turning counts at these three intersections. The quality of the calibration was evaluated based on the mean absolute error (MAE) and mean absolute percentage error (MAPE). Equations 5-1 and 5-2 show the expression for these measures.

$$
\begin{gathered}
M A E=\frac{1}{N} \sum_{t}\left|P_{t}-P_{t, a}\right| \\
M A P E=100 \frac{1}{N} \sum_{t} \frac{\left|P_{t}-P_{t, a}\right|}{P_{t, a}}
\end{gathered}
$$

where

$$
P_{t}=\text { estimated turning movement volume per } 15 \text { minutes, }
$$




$$
\begin{aligned}
& P_{t, a}=\text { corresponding real-world data measurement, and } \\
& N=\text { total number of the time intervals. }
\end{aligned}
$$

Figures 5-2, 5-3 and 5-4 show MAE (upper numbers) and MAPE (lower numbers) for each movement and for three demand levels. It is seen from these figures that the dynamic ODME process with the utilizing of the turning movement as input can produce acceptable simulated turning movement counts. Since the right turn and left turn counts usually are less than the through movement counts, the MAPE for these movements are more than MAPE of through movements. However, the MAE values, which are related to the volume amounts, show that there is an acceptable discrepancy between the observed and simulated counts of the right turn and left turn movements.
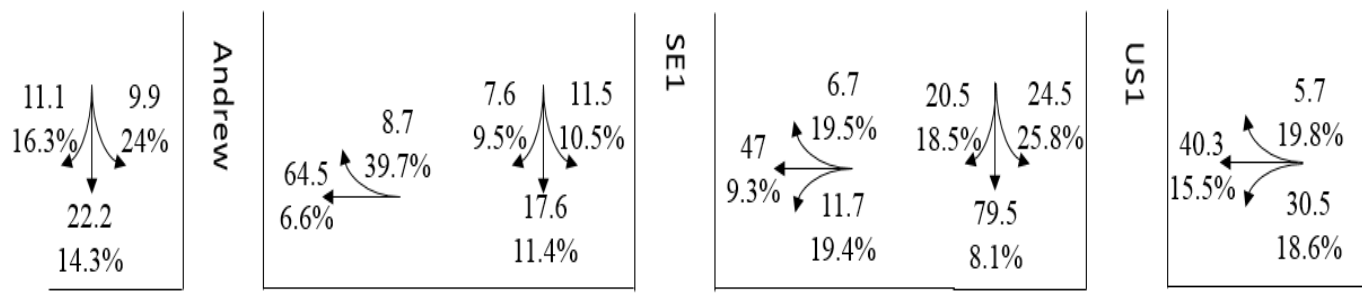

Broward Blvd

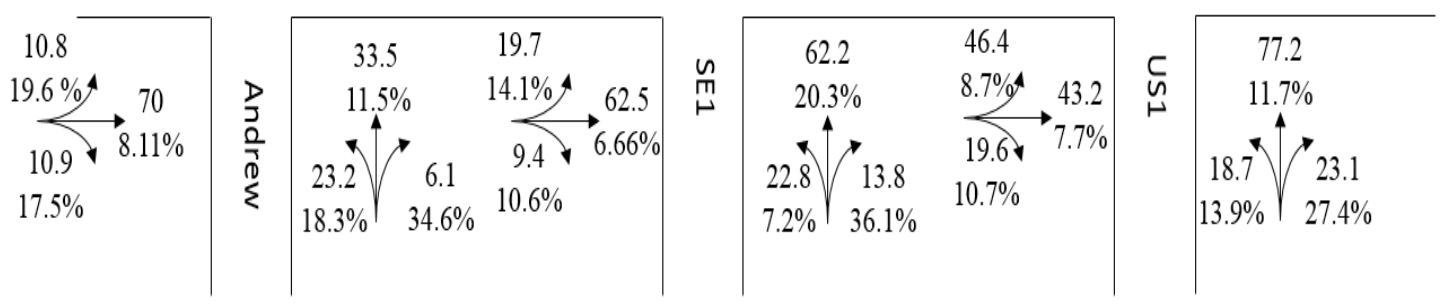

Figure 5-2 MAE (Upper Numbers) and MAPE (Lower Numbers) for Each Left, Through and Right Turning Movements for Day with 50th Percentile of Demand 


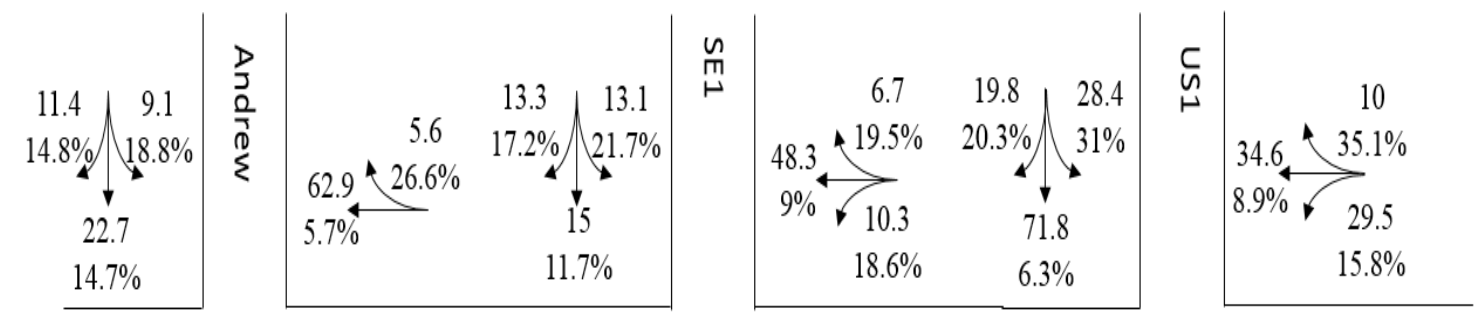

Broward Blvd

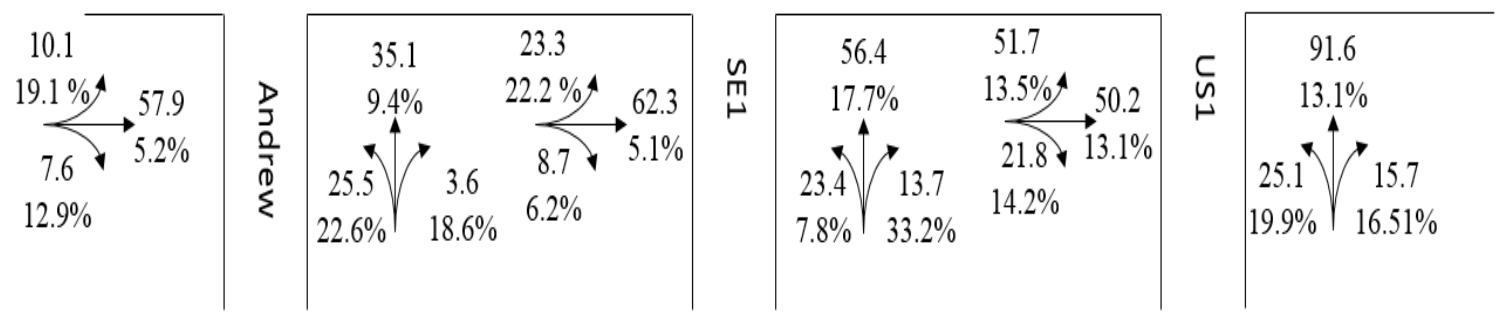

Figure 5-3 MAE (Upper Numbers) and MAPE (Lower Numbers) for Each Left, Through and Right Tirning Movements for Day with $75^{\text {th }}$ Percentile of Demand
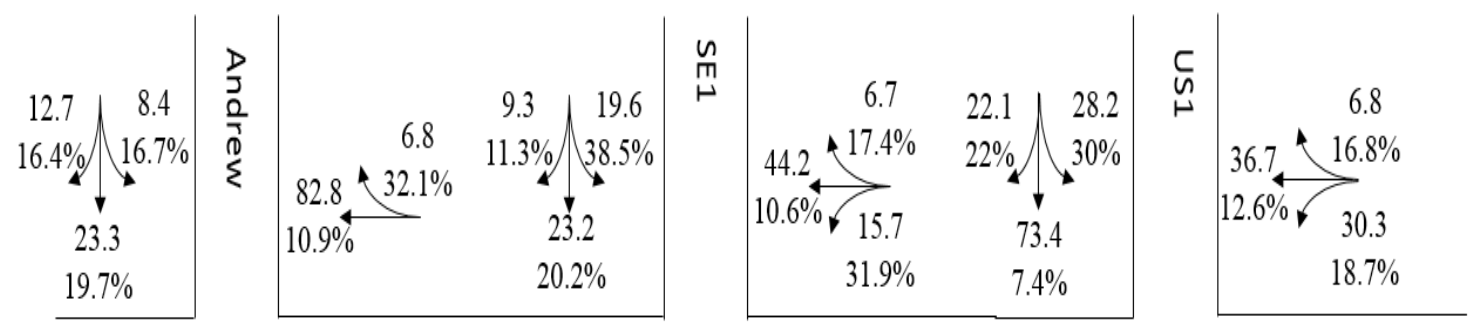

Broward Blvd
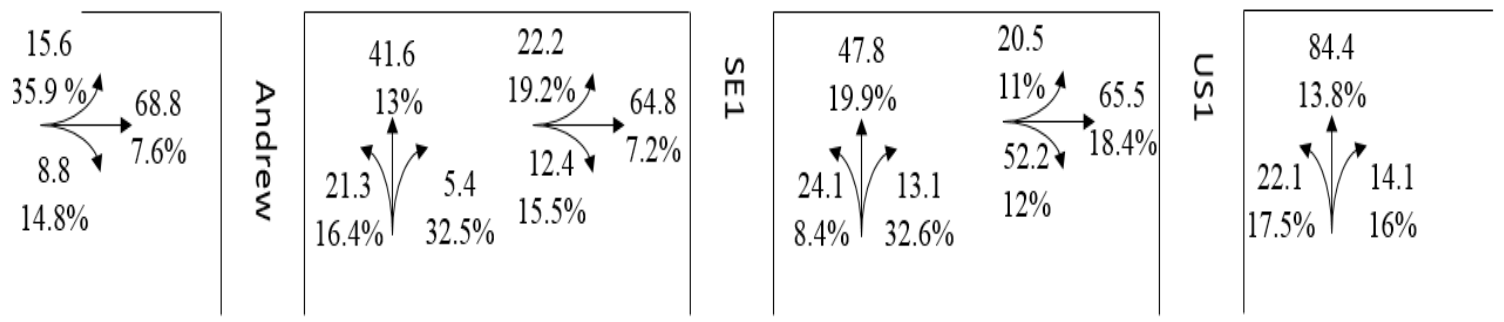

Figure 5-4 MAE (Upper Numbers) and MAPE (Lower Numbers) for Each Left, Through and Right Turning Movements for Day with $95^{\text {th }}$ Percentile of Demand 


\subsection{HCS Signal Optimization on Network Performance}

Before the construction of the network in the VISSIM, the network coded in the HCS was designed and matched with the VISSIM network to allow a valid comparison. The calibrated VISSIM model produced a saturation flow rate of $1854 \mathrm{veh} / \mathrm{hr} / \mathrm{lane}$, which was used as the input saturation flow model in the HCS. The base analysis period in the HCM, and thus HCS analysis, is fixed at 15 minutes. Once the network was coded in the HCS, a full optimization with 200 iterations were conducted to minimize the network total delay. This result is the optimized pre-timed signal settings; in terms of offset, cycle length and green split; however, the phase pattern and sequence are assumed to be fixed. Signal optimization were run for different traffic patterns including $50^{\text {th }}, 75^{\text {th }}, 95^{\text {th }}$ percentile volumes of normal days during the PM peak period and the resulting signal timings were used in the multi-resolution analysis.

\subsection{Network Modeling in Microscopic Simulation}

The VISSIM microscopic simulation tool was used as part of the multi-resolution framework of this study. Before evaluating the impact of signal optimization on the network performance, it is necessary to calibrate the model based on real-world data to reflect measured traffic parameters for the utilized network.

\subsubsection{VISSIM Model Calibration}

VISSIM was used in this study to further assess the performance of the signal timing plans developed to emulate demand responsive signal control, as described in the previous section. In order to accurately reflect the real-world traffic conditions in the 
VISSIM, the network needs to be calibrated to reflect the saturation flow rate estimated for the study area.

The saturation flow rate in VISSIM was calibrated in a hypothetical network. Since the saturation flow rate of an approach is defined as the maximum number of vehicles that can pass through the intersection during one hour (HCM 2010), no signal interruption and no conflicting traffic were allowed to affect the vehicle movements in the calibration of the saturation flow rate. Hence, in this scenario, a large number of vehicles were fed to one direction and the intersection signal control was set to serve green indication for this direction in the whole studied time period (i.e., an hour). The volume input at the upstream of the intersection was manipulated to be high enough to guarantee that the upstream demand was higher than the volume count at the immediate downstream of the intersection. In this case, the volume count at the immediate downstream of the intersection was extracted as the saturation flow rate.

VISSIM urban driver behavior model for motorized vehicles adopts the Wiedemann 74 driver behavior model. Based on the above literature review, two parameters were investigated in this study to obtain the reasonable saturation flow rate: 1) additive part of desired safety distance (bx_add) and 2) multiplicative part of desired safety distance (bx_mult) (Kim et al., 2005; AG 2007; Al-Nuaimi et al., 2013). The reason for examining the aforementioned two parameters is that they determine the target desired safety distance, which has a direct impact on saturation flow rate. The saturation flow rate in number of vehicles per hour per lane decreases with the increase of these two parameters. 
According to the HCM 2010 (HCM 2010), the ideal saturation flow rate is 1,900 passenger car per hour per lane for signalized intersections in a metropolitan area with population higher than 250,000 . Therefore, the target saturation flow rate for the purpose of this calibration is set to be 1,900 passenger car per hour per lane. It can be inferred from Figure 5-5 that the second parameter, (i.e., multiplicative part of desired safety distance) should be higher than 3.00 but lower than 3.50. The first parameter (i.e., the additive part of desired safety distance) ranges from 2.00 to 2.50 , respectively.

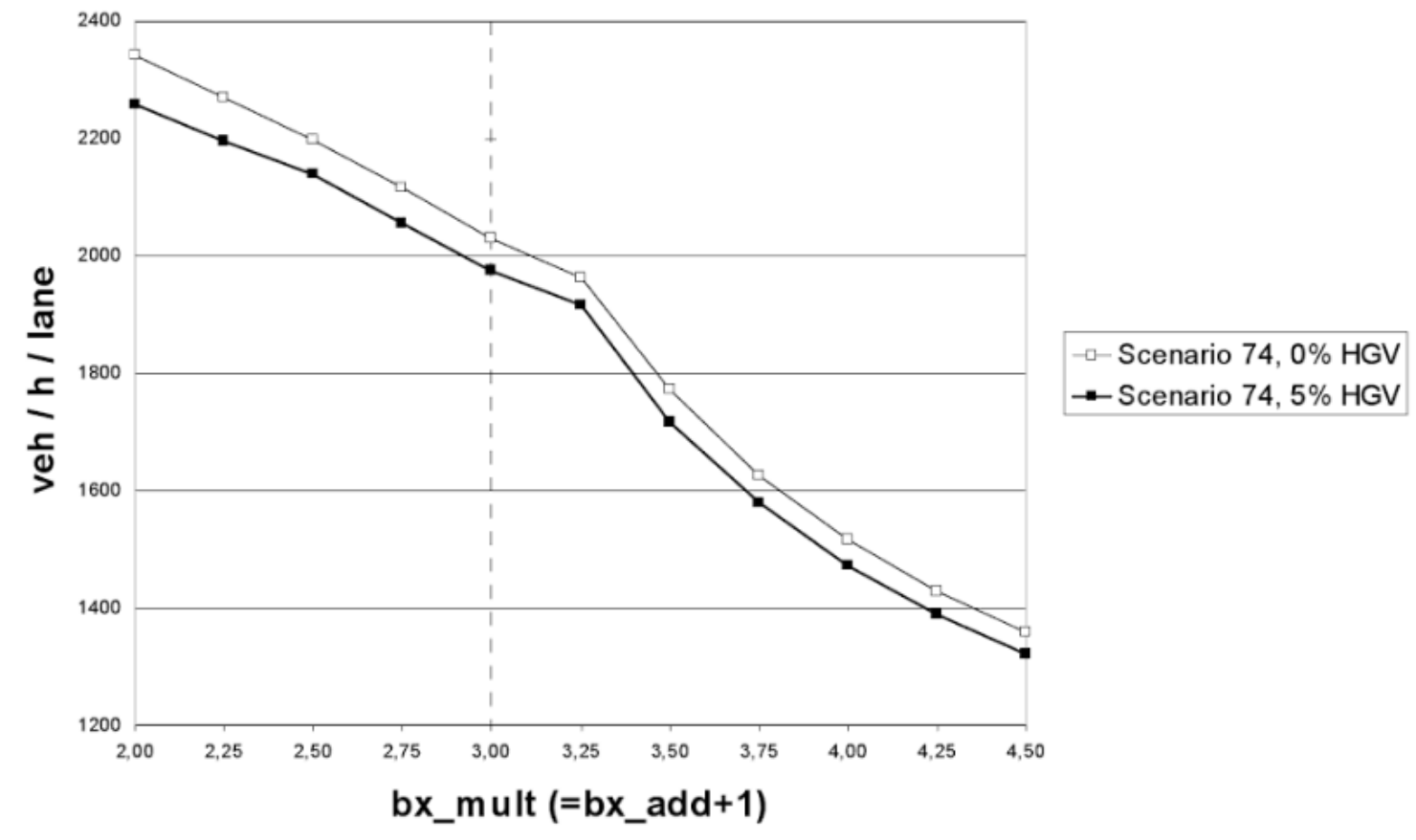

Figure 5-5 Relationship Between Saturation Flow Rate and Desired Safety Distance Parameters (Oregon 2011)

Based on the discussion above, the two parameters of the desired safety distance were adjusted manually within the specified ranges and the difference between them was kept as 1 . The most appropriate combination of the two parameters was found to be 2.4 
feet for the additive part of desired safety distance and 3.4 feet for the multiplicative part of desired safety distance, while the default values are 2 feet and 3 feet for those two parameters, respectively. The saturation flow rate for the simulated direction was 1,854 passenger cars per hour per lane, which was the average number of ten simulation runs with various random seeds. The modeled saturation flow rate is close to the recommended value by HCM (i.e., 1,900 passenger cars per hour per lane) and similar to the findings from the literature (HCM 2010).

Table 5-1 Default and Calibrated Parameters

\begin{tabular}{|c|c|c|}
\hline Parameter & Default Value & Calibration Value \\
\hline Look Back Distance & $\begin{array}{c}\text { Minimum Value: } 0 \\
\text { Maximum Value: } 820.21 \mathrm{ft} .\end{array}$ & $\begin{array}{c}\text { Minimum Value: } 25 \mathrm{ft} . \\
\text { Maximum Value: } 820.21 \mathrm{ft}\end{array}$ \\
\hline Look Ahead Distance & $\begin{array}{c}\text { Minimum Value: } 0 \\
\text { Maximum Value }: 820.21 \mathrm{ft} .\end{array}$ & $\begin{array}{c}\text { Minimum Value: } 25 \mathrm{ft} . \\
\text { Maximum Value }: 820.21 \mathrm{ft} .\end{array}$ \\
\hline Average Standstill Distance & $6.56 \mathrm{ft}$. & $6.56 \mathrm{ft}$. \\
\hline $\begin{array}{c}\text { Additive Portions of the } \\
\text { Safety Distance Factor }\end{array}$ & $2 \mathrm{ft}$. & $2.4 \mathrm{ft}$. \\
\hline $\begin{array}{c}\text { Multiplicative Portions of } \\
\text { the Safety Distance Factor }\end{array}$ & $3 \mathrm{ft}$. & $3.4 \mathrm{ft}$. \\
\hline
\end{tabular}

\subsubsection{Volume Representation in VISSIM}

In this section, we investigate the impact of utilizing two different methods of inputting the volumes estimated by the mesoscopic simulation tool to the VISSIM microscopic simulation tool. The VISSIM software accepts two methods of inputting traffic movement demands. The first involves inputting "partial routes" and the second involves inputting turning movement counts. Usually, turning movement counts are coded for urban arterials, as it is difficult to estimate the partial paths of the vehicle, which 
represent a part of the route of the vehicles between their origins and destinations. The partial route coding, however, is expected to better replicate real-world conditions. Results from mesoscopic simulation-based DTA tools provide the required information to input the partial routes to the microscopic simulation. The impact of utilizing the partial routes compared to turning movement counts was investigated in this study. A segment of Broward Boulevard in Broward County was used as a case study. The segment is bounded by US-1 to the east and Andrews Avenue to the east, as shown in Figure 5-6.

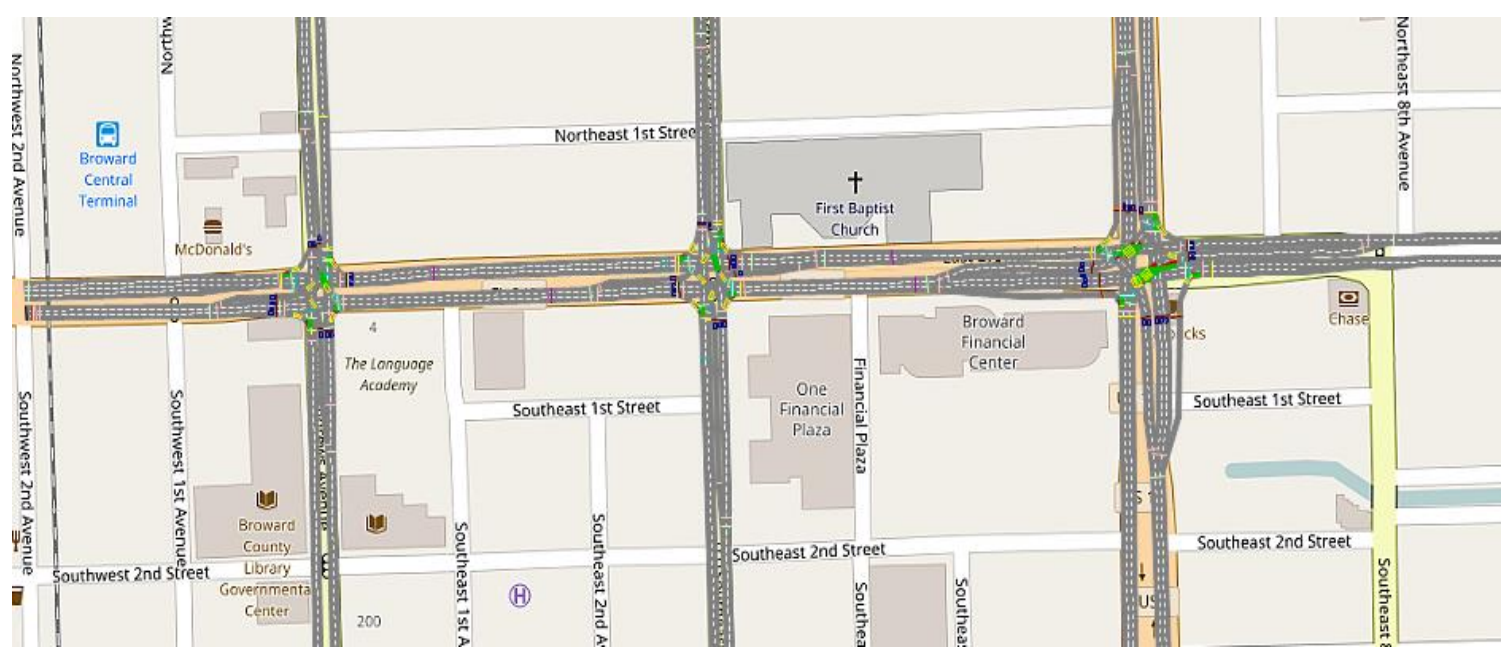

Figure 5-6 Case Study Network 


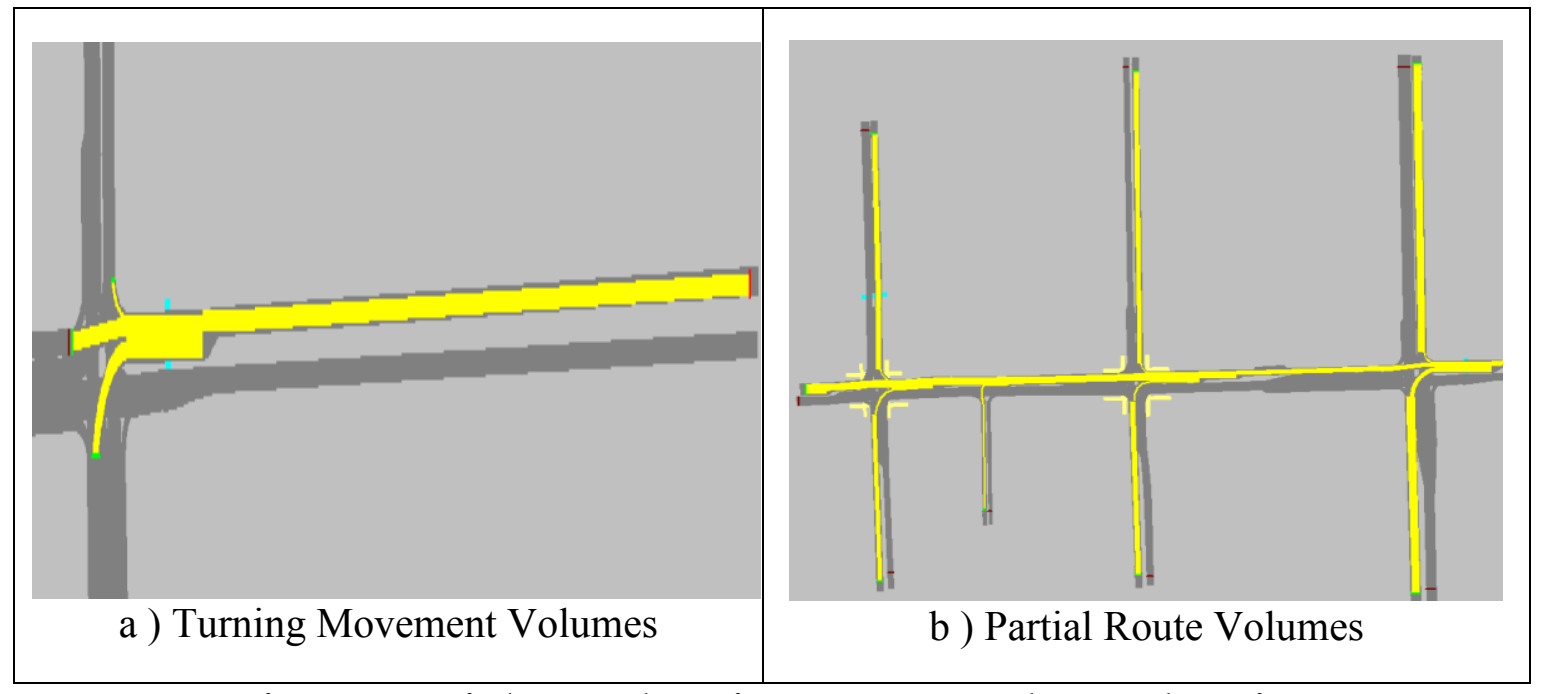

Figure 5-7 Link Based Assignment vs. Path Based Assignment

The traffic volumes output from the DTAlite tool were input to VISSIM. Figure 5-7 illustrate the two methods mentioned above of inputting volumes to VISSIM. To compare the impact of utilizing the partial route volumes instead of turning movement volumes, the travel time and delay outputs from the VISSIM models with the two methods were compared for both direction of the main street (Broward Boulevard). As can be seen in Figure 5-8, the travel time and delay estimates when utilizing partial routes were significantly different than those when coding turning movement counts for all travel demand intervals. To compare the network performance under the two methods, the total delay per vehicle and the network total travel time for the three demand levels including medium, heavy and very heavy days are presented in Figure 5-9. As expected, the very high and high demand days have more total travel time and, consequently, they produced more delays compared with medium demand day. It also can be seen the modeling with 
partial routes produced more delays and, subsequently, more travel time compared to the link based method for the modeled three demand levels.
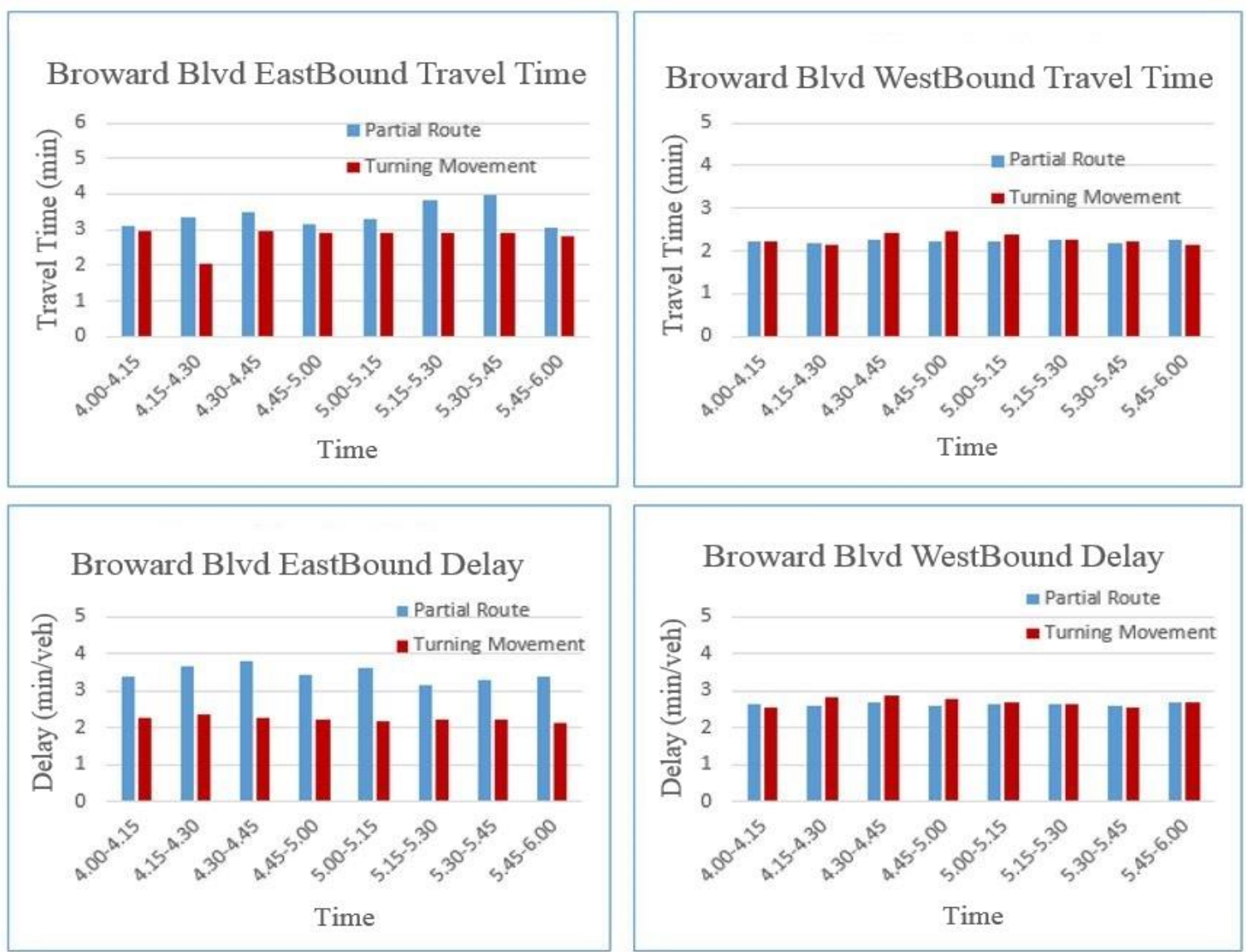

Figure 5-8 Comparison Between Path Based Assignment and Link Based Assignment for the Median (50\%) Demand Day 


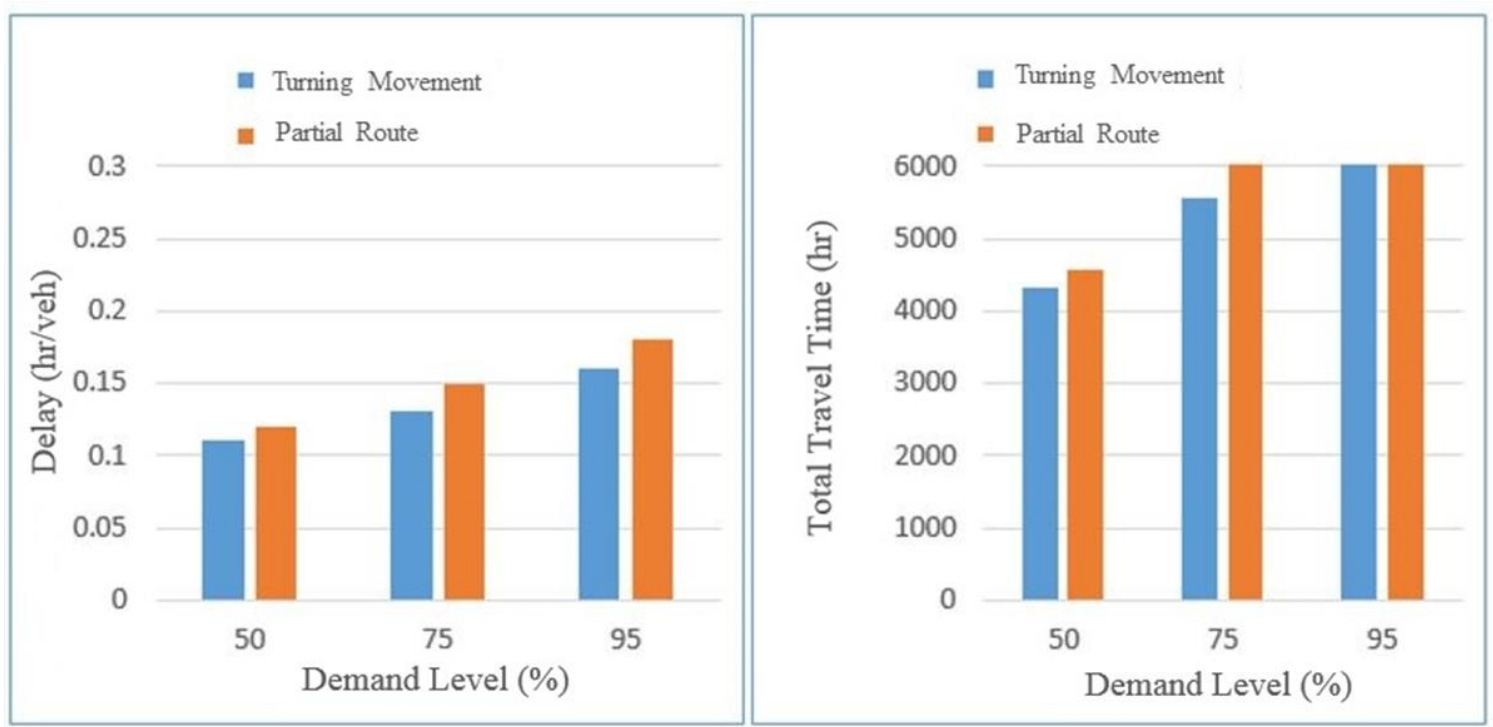

Figure 5-9 Comparison of Delay and Travel Time Between Path Based Assignment and Link Based Assignment

\subsection{Microscopic Simulation Assessment of Demand Responsive Signal Control}

This section describes the method used in the assessment of demand responsive signal control and the obtained results. Two levels of dynamic signal control plans were tested. At the first level, a plan is selected from a library of plans for the specific day under consideration, depending on the volume level but the plan is kept fixed during the whole period. The second level is more responsive control that allows changing the plans with the change in demands during the period. To simplify the analysis, the model changes the plan every 15 minutes, although the real-world adaptive control can change the plans at more frequent intervals.

For the performance evaluation of the first level signal control, three test scenarios were prepared including very high demand $\left(95^{\text {th }}\right.$ percentile $)$, high demand $\left(75^{\text {th }}\right.$ percentile), and medium demand $\left(50^{\text {th }}\right.$ percentile) levels. With conventional time-of-day control, the 
signal control plan was assumed to be derived for the medium day and applied for days with different demand control. Thus, to model the time of control, the signal controls was optimized for the $50^{\text {th }}$ volumes and applied for the three scenarios. For the first level of traffic signal control, the signals are optimized with the demands of the three scenarios $\left(50^{\text {th }}, 75^{\text {th }}\right.$, and $95^{\text {th }}$ percentile demands $)$.

As mentioned above, the travel demand for the case study segment was obtained using the mesoscopic analysis tool and used as an input to the VISSIM model. As shown in Table 5-2, that total network delay decreased from 6,475 to 6,121 vehicle-hours, if the signal was optimized for the $75^{\text {th }}$ percentile demand level, meaning that selecting a plan that was designed for this level of demand rather than using a plan developed for the median demand resulted in a saving of about 354 vehicle-hours of delay (5\%) for the peak hour of the three modeled intersections when evaluated using VISSIM. [When evaluated using the HCS, the delay saving was 295 vehicle-hours].

The impact of optimizing the signal control for the $95^{\text {th }}$ percentile demand level on the mobility results are presented in Table 5-3. As shown in this Table, the network total travel delay was lowered by 253 vehicle-hours (about $2.8 \%$ ) when the traffic signals were optimized for $95^{\text {th }}$ demand level when evaluated using VISSIM. [363 vehicle-hours when evaluated using the HCS]. 
Table 5-2 The Performance of Optimizing Control Plan for the $75^{\text {th }}$ Percentile of Demand Level vs. Using the Optimized Plan for the Median Day

\begin{tabular}{|c|c|c|c|}
\hline Street & $\begin{array}{c}\text { Optimized } \\
\text { Signal Delay } \\
\text { (veh-hrs) }\end{array}$ & $\begin{array}{c}\text { Optimized Traffic } \\
\text { Signal for Median Day } \\
\text { Delay (veh-hrs) }\end{array}$ & $\begin{array}{c}\text { Delay Saving } \\
\text { (veh-hrs) }\end{array}$ \\
\hline Network & 6121 & 6475 & 354 \\
\hline Broward EB & 1106 & 1533 & 427 \\
\hline Broward WB & 1096 & 1267 & 171 \\
\hline N Andrews NB & 295 & 300 & 5 \\
\hline N Andrews SB & 242 & 259 & 16 \\
\hline NE 3 rd. AVE NB & 540 & 428 & -112 \\
\hline NE 3 rd. AVE SB & 253 & 222 & -30 \\
\hline US1 NB & 693 & 614 & -78 \\
\hline US1 SB & 675 & 624 & -52 \\
\hline
\end{tabular}

Table 5-3 The Performance of Optimizing Control Plan for the $95^{\text {th }}$ Demand Level vs. Using the Optimized Plan for the Median Day

\begin{tabular}{|c|c|c|c|}
\hline Street & $\begin{array}{c}\text { Optimized Signal } \\
\text { Delay (veh-hrs) }\end{array}$ & $\begin{array}{c}\text { Optimized Traffic } \\
\text { Signal for Median Day } \\
\text { Delay (veh-hrs) }\end{array}$ & $\begin{array}{c}\text { Delay Saving } \\
\text { (veh-hrs) }\end{array}$ \\
\hline Network & 9036 & 9290 & 253 \\
\hline Broward EB & 1539 & 2115 & 577 \\
\hline Broward WB & 1580 & 1522 & -58 \\
\hline N Andrews NB & 383 & 810 & 428 \\
\hline N Andrews SB & 311 & 267 & -45 \\
\hline NE 3 rd. AVE NB & 1064 & 445 & -619 \\
\hline NE 3 rd. AVE SB & 297 & 244 & -53 \\
\hline US1 NB & 756 & 662 & -94 \\
\hline US1 SB & 962 & 1028 & 66 \\
\hline
\end{tabular}

The second level (more dynamic) traffic responsive signal control was evaluated by defining 8 traffic signal timing patterns during the PM peak period (4:00 to 6:00 PM). Each pattern was optimized for the demands of the corresponding 15 minutes of the PM peak period using the HCS software. As shown in Table 5-4, the network mobility 
improved by 387 vehicle-hours (6.4\%) for the $75^{\text {th }}$ percentile demand level. By comparing Table 5-2 and 5-4, it can be concluded that the delay saving for whole network changed from 354 to 387 vehicle-hours. Whereas, delay saving for main street (Broward) EB and WB changed from 427 and 171 to 342 and 213 vehicle-hours. However, there was a significant saving in delay for the cross streets, when using the more dynamic traffic signal control.

Table 5-4 The Benefits of the More Dynamic Responsive Control for the $\mathbf{7 5}^{\text {th }}$ Percentile of Demand

\begin{tabular}{|c|c|c|c|}
\hline Street & $\begin{array}{c}\text { Optimized } \\
\text { Signal Delay } \\
\text { (veh-hrs) }\end{array}$ & $\begin{array}{c}\text { Optimized Traffic Signal } \\
\text { for Median Day } \\
\text { Delay (veh-hrs) }\end{array}$ & $\begin{array}{c}\text { Delay Saving } \\
\text { (veh-hrs) }\end{array}$ \\
\hline Network & 6088 & 6475 & 387 \\
\hline Broward EB & 1191 & 1533 & 342 \\
\hline Broward WB & 1054 & 1267 & 213 \\
\hline N Andrews NB & 310 & 300 & -10 \\
\hline N Andrews SB & 231 & 259 & 27 \\
\hline NE 3 rd. AVE NB & 495 & 428 & -67 \\
\hline NE 3 rd. AVE SB & 233 & 222 & -10 \\
\hline US1 NB & 679 & 614 & -64 \\
\hline US1 SB & 676 & 624 & -53 \\
\hline
\end{tabular}

Comparing Tables 5-3 and 5-5 indicates that the network travel delay saving increased from 253 to 380 vehicle-hours ( $2.8 \%$ to $4.2 \%)$, when using the more dynamic traffic response signal control for the $95^{\text {th }}$ percentile demand. It can be also concluded that delay saving for the main street (Broward) EB decreased from 577 to 207 vehicle-hours. However, there is a relatively significant saving in delay for the cross street movements. 
Table 5-5 The Benefits of the More Dynamic Responsive Control for the $\mathbf{9 5}^{\text {th }}$ Percentile of Demand

\begin{tabular}{|c|c|c|c|}
\hline Street & $\begin{array}{c}\text { Optimized } \\
\text { Signal Delay } \\
\text { (veh-hrs) }\end{array}$ & $\begin{array}{c}\text { Optimized Traffic Signal } \\
\text { for Median Day } \\
\text { Delay (veh-hrs) }\end{array}$ & $\begin{array}{c}\text { Delay Saving } \\
\text { (veh-hrs) }\end{array}$ \\
\hline Network & 9036 & 9290 & 380 \\
\hline Broward EB & 1909 & 2115 & 207 \\
\hline Broward WB & 1562 & 1522 & -40 \\
\hline N Andrews NB & 406 & 810 & 405 \\
\hline N Andrews SB & 301 & 267 & -35 \\
\hline NE 3 rd. AVE NB & 655 & 445 & -210 \\
\hline NE 3 rd. AVE SB & 197 & 244 & 47 \\
\hline US1 NB & 736 & 662 & -74 \\
\hline US1 SB & 943 & 1028 & 85 \\
\hline
\end{tabular}

\subsection{Summary}

An analysis of the segment level calibration results indicated that this level of calibration is not adequate to produce good turning movement volumes. The case study used in this dissertation was further calibrated at the turning movement level. The microscopic simulation model network was prepared and the network maximum saturation flow rate was calibrated by adjusting the VISSIM urban driver behavior model. The demands generated from the utilized DTA tool was input into the signal timing optimization tool and microscopic simulation tool to assess demand responsive signal control. For the performance evaluation, three test scenarios were prepared including very high demand ( $95^{\text {th }}$ percentile), high demand $\left(75^{\text {th }}\right.$ percentile), and medium demand $\left(50^{\text {th }}\right.$ percentile) levels. Two levels of traffic responsive signal controls were tested. In the first level, a signal control plan is selected from a library of plan for the specific day depending on the volume level but the plan was kept fixed during the whole period. The second level 
is more responsive control that allows changing the plans with the change in demands during the period (15 minutes). The results indicate that the delay resulting from the implementation the traffic responsive signal control strategies can be reduced significantly, and that this method can be used as part of planning for operations to evaluate and design strategies. 


\section{CHAPTER 6}

\section{INCIDENT RESPONSE SIGNAL CONTROL}

\subsection{Introduction}

Incident management is a vital part of ATM strategies. Estimating the impact of incidents and incident management allows traffic management agencies to determine the need for various incident management strategies and technologies to justify the decision to invest in their programs. These strategies include incident detection, response, and clearance strategies and techniques. Special signal control plans during incident conditions are critical components of arterial incident management strategies. Special signal control plans can increase roadway capacity during arterial incidents and diversion due to freeway incidents. In addition, modifying signal timing can be combined with traveler information that guides motorists to alternative routes (Turnbull 2005). With an emphasis on implementing incident management strategies for signalized urban streets in recent years, there has been an increasing interest in models that estimate incident impacts on these streets. Such estimation will allow agencies to determine the need for special signal timing plans and other management strategies during incident conditions. This chapter describes a new approach to estimate the capacity impacts due to arterial incidents that result from the interaction between the drop in capacity below demands at midblock urban street segment locations and upstream and downstream signalized intersection operations. Models were developed in this study based on data generated utilizing a microscopic simulation model that was calibrated by a comparison of capacity values suggested in the 
HCM and SHRP2-L08 project for incident and no-incident conditions, and by a comparison of field measurements of system performance. These developed models can be used as part of the macroscopic and mesoscopic analysis models. In this study, the developed models are used to set the capacity during the incident conditions in the mesoscopic-based DTA tool (DTAlite) to support the estimation of the diversion during incidents. To supplement the DTA analysis, regression models were developed to estimate the diversion rate due to urban street incidents. These regression models were combined with the DTA model to estimate the volume at the incident location and alternative routes. The developed models will assist agencies in estimating incident impacts on urban street facilities and support the development and use of special signal plans based on incident attributes and urban network characteristics. This chapter also describes the development and application of a new model that can estimate the impacts of incidents and incident management strategies on urban street facilities. The developed method was successfully used to estimate the impact of modifying signal timing plans during incidents with consideration of the estimating drop in capacity.

\subsection{Estimating the Capacity Impacts of Urban Street Incidents}

An incident occurring at mid-block locations can decrease the throughput of the incident link and upstream and downstream intersections. Upstream intersection throughput capacity is affected when the queue from the incident spills back to the upstream links. During the first part of the upstream link green phases, the vehicles are able to leave the stop lines of the feeding links at the saturation flow rates of these links 
until the queue due to the downstream incident spillbacks to the upstream signal. From the moment of this occurrence until the end of the green, the throughput of the upstream links will be controlled by the allowable throughput at the downstream incident location. Thus, the upstream movement greens can be divided into two parts. The first part, referred to as the "unconstrained green" in this study, is the green time portion that vehicles from the upstream links can leave at the throughput of upstream links due to the availability of queuing storage at the downstream link. In the second part, referred to as the "constrained green," the movements from the upstream links are controlled by the capacity at the incident location due to the spillback of the queues from the downstream incident. The result of having this constrained green is a reduction in the capacity of the upstream intersection feeding links, which causes an increase in the upstream movement delays. The unconstrained green portion is expected to increase as the incident is located further downstream from the stop line. In addition, higher reductions in incident capacity and higher demands at the incident location will result in an increase in the constrained green of the upstream intersection. It should be noted that this only occurs when there is a spillback from the incident location to the upstream signal.

On the other hand, incident link capacity can be affected by the downstream intersection queue if it spills back to the upstream incident location. In addition, a reduction in link capacity due to incidents can prevent vehicles from arriving at downstream signalized intersections, reducing the utilization of downstream green movement and thus the capacity. Consequently, this decreases downstream intersection queue discharges. 
The reduction in the upstream intersection maximum throughputs is expected to be a function of how far the incident is from the upstream intersection, the volume to capacity $(\mathrm{v} / \mathrm{c})$ ratio at the incident location, and incident duration. Whereas the capacity at the incident location (IC) is expected to be a function that determines how far the incident is from the downstream intersection, and also determine its green to cycle-length $(\mathrm{g} / \mathrm{C})$ ratio and number of total and blocked lanes. This study developed two models to estimate the impacts of incidents on capacity, taking into consideration the interactions with upstream and downstream signalized intersections. The first model estimates the remaining capacity of the incident link with lane-blockage incidents located at various distances from the downstream signal. This model considers the impact of the interaction between downstream signal operation and the drop in the capacity at the incident location. It is a function of the $\mathrm{g} / \mathrm{C}$ ratio of the downstream signal, distance of the incident to the downstream intersection, and the number of the blocked lanes due to the incident. The second model estimates the reduction in the upstream intersection maximum throughput as a result of the incidents at different downstream locations. This model is a function of the volume to capacity $(\mathrm{v} / \mathrm{Ic})$ ratios at the incident location, distance to the incident, and incident duration times. The two models can be used together to estimate the delays due to incidents depending on the drop in capacity at the upstream signal due to queue spillback from the incident locations. 


\subsubsection{Procedure of Estimating Incident Capacity in Urban Street}

As stated above, this study estimates the interaction between incidents and upstream and downstream signalized intersections on capacity. The first step is to estimate the drop in link capacity due to incidents without this interaction. This estimation was conducted in this research based on the SHRP2 Program L08 project (Zegeer et al., 2014) and the HCM 2010 recommendations to estimate the remaining link capacity due to incidents at midblock locations. The SHRP2 L08 equations can only be used to estimate the capacity drop due to incidents at the movement stop lines (Zegeer et al., 2014). This study uses these abovementioned values to estimate the throughput at an incident midblock location, calibrate a microscopic simulation model to produce the capacity at the incident location, and examine the interactions with upstream and downstream signals using microscopic simulation to develop the models, as described in this section.

\subsubsection{Calibration of Microscopic Simulation for No-Incident Conditions}

In order to develop the abovementioned models, the study used the VISSIM microscopic simulation tool. The model was first calibrated for no-incident conditions based on real-world data to reflect measured traffic parameters for the utilized network. The manual calibration process was divided into two parts. The first is based on saturation flow rate, and the second is based on traffic flow performance measures. A three-mile segment of Glades Road, an urban street in Boca Raton, Florida, was used as a case study. The segment has nine signalized intersections with an average signal spacing of 1,760 feet. The posted speed limit is 45 miles per hour. The Glades Road network was coded in 
VISSIM, as shown in Figure 6-1, and the results from the simulation were compared with real-world measurements.

The first step was to perform an initial fine-tuning of the model's parameters to produce saturation flow rates that are in agreement with the Highway Capacity Manual 2010 (HCM 2010) procedures (HCM 2010) and previous observations from the field in South Florida. The target saturation flow rate for the purpose of this calibration was set from 1,850 to 1,900 passenger cars per hour per lane. The parameters of the VISSIM model were fine-tuned to produce this value.

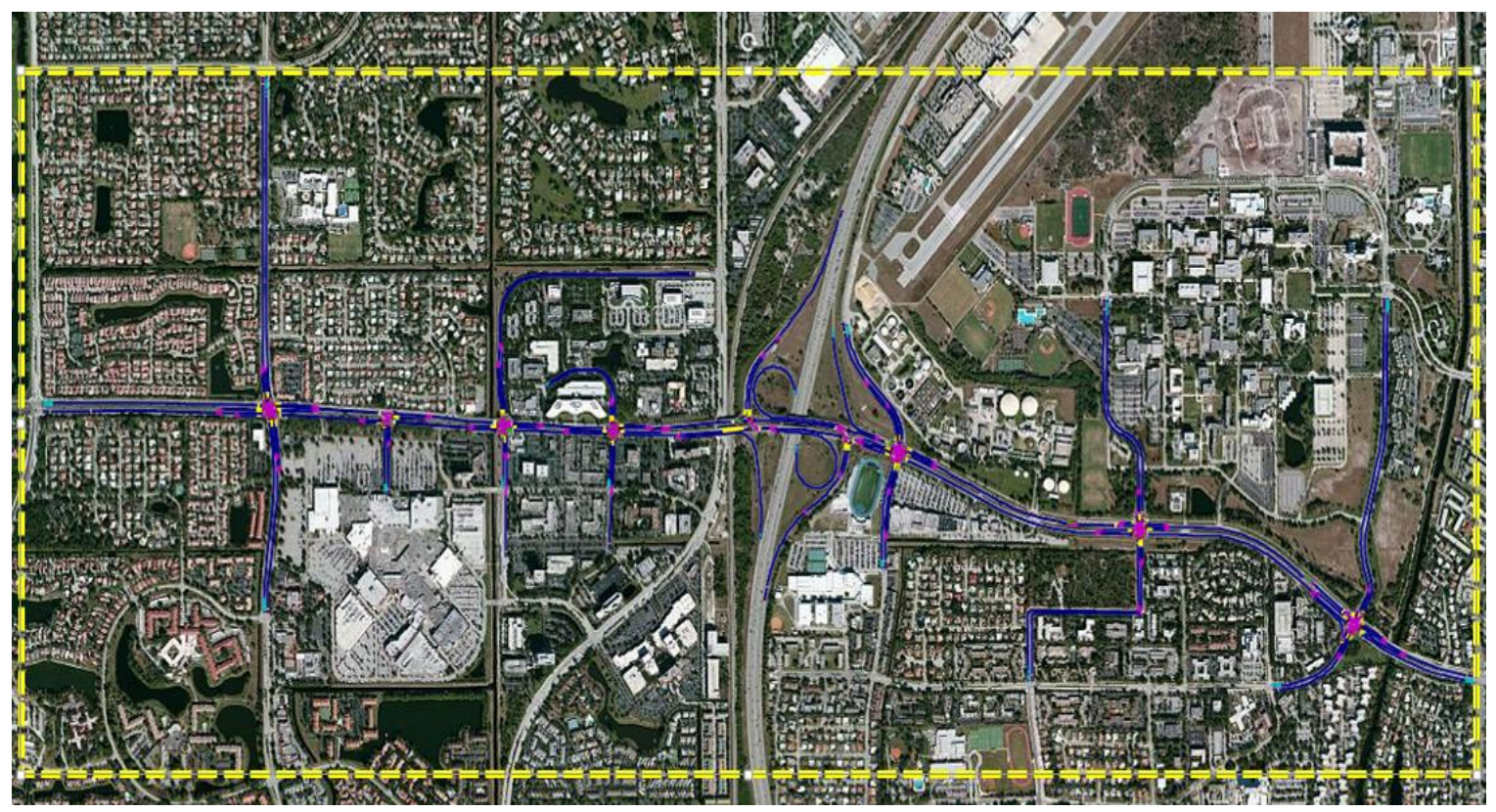
Figure 6-1 Study Network

Two of the urban driver car-following models in VISSIM (Wiedemann 74 driver behavior model) were fine-tuned in this study in order to obtain the target saturation flow rate: 1) the additive part of desired safety distance (bx_add); and 2) the multiplicative part of the desired safety distance (bx_mult) (Kim et al., 2005; AG 2007; Al-Nuaimi et al., 
2013). These parameters determine the desired safety distance, which has a direct impact on saturation flow rate (26). The most appropriate combination of the two parameters was found to be 2.4 feet for the additive part of the desired safety distance and 3.4 for the multiplicative part of the desired safety distance. The resulting saturation flow rate for the simulated thru movement was 1,854 passenger cars per hour per lane based on the average of ten simulation runs with different random seeds.

The second part of the calibration process compared the traffic flow performance according to the Glades Road simulation model with real-world traffic data. Three traffic data types were used in the calibration, including: 1) signal timing plans of all intersections; 2) historical turning movement counts of all intersections; and 3) data from permanently installed magnetometers (from SENSYS). These data types provide point measurements of speed, occupancy, and volume, as well as travel time measurements based on automatic vehicle re-identification. Virtual detectors were included in the simulation at the same locations of the detectors in the field. The measured and simulated parameters based on ten simulation runs with different seed numbers were examined to determine if further finetuning of the parameters was needed. It was determined that the Percentage-Root-MeanSquare-Errors (PRMSE) for the simulated versus measured volume and speed values were below $15 \%$ for both directions of travel.

\subsubsection{Estimating Capacity Drop due to Incidents at the Movement Stop Lines}

Once the network was calibrated for no-incident conditions, it was necessary to model the incident conditions. In order to model incident impacts using simulation, it was 
necessary to emulate the estimated drop in capacity at the incident location in the simulation model. The capacity drop at the incident location was estimated based on the equations developed in the SHRP2 Program L08 project referenced earlier (Zegeer et al., 2014). The equations used in the estimation is as follows:

$$
f_{i c, \operatorname{int}(i), n, m}=\left(1-\frac{N_{i c, i n t(i), n, m}}{N_{n, \operatorname{int}(i), n, m}}\right)\left(1-\frac{b_{i c, \operatorname{int}(i), n}}{\sum_{m \in L, T, R} N_{n, i n t(i), n, m}}\right) \geq 0.01
$$

with

$$
\mathrm{b}_{i c, \operatorname{int}(i), n, m}=0.58 I_{f i, \operatorname{int}(i), n}+0.42 I_{\text {pdo,int }(i), n}+0.17 I_{\text {other,int }(i), n}
$$

where

$$
\begin{aligned}
& f_{i c, \text { int }(i), n, m, a p, d}=\text { saturation flow adjustment factor for incident influence on } \\
& \text { movement } \mathrm{m}(\mathrm{m}=\mathrm{L} \text { : left, } \mathrm{T} \text { : through, } \mathrm{R} \text { : right }) \text { at intersection } \mathrm{i} \text {, } \\
& N_{n, \text { int }(i), n, m}=\text { number of serving movement lanes (m) on leg associated } \\
& \text { with phase (n) at intersection i, } \\
& N_{i c, \text { int }(i), n, m, a p, d}=\text { number of serving movement lanes (m) blocked by the incident } \\
& \text { on leg associated with phase (n) at intersection i, } \\
& b_{i c, \text { int }(i), n}=\text { calibration coefficient on leg associated with phase (n) at } \\
& \text { intersection i, } \\
& I_{f i, \text { int }(i), n} \quad=\text { indicator variables for fatal-or-injury on leg associated with } \\
& \text { phase (n) at intersection i, } \\
& I_{p d o, \text { int }(i), n}=\text { indicator variables for property damage only (PDO) on leg } \\
& \text { associated with phase (n) at intersection } \mathrm{i} \text {, and }
\end{aligned}
$$




$$
\begin{aligned}
I_{\text {other,int }(i), n=} \quad & \text { indicator variables for non-crash incident on leg associated } \\
& \text { with phase (n) at intersection i. }
\end{aligned}
$$

\subsubsection{Modeling Incidents in Microscopic Simulation}

This section describes the calibration of microscopic simulation models for incident conditions in VISSIM. VISSIM does not allow the user to specify incidents in the model. Previous studies modeled incidents by setting up a red signal at the incident lane using vehicle(s) with zero speed at the time and location of the incident (Avetisyan et al., 2014) or using buses with dwelling times equal to the lane blockage durations (Hadi et al., 2007). In this study, the incidents were simulated in VISSIM using bus stops with dwell times equal to incident duration on the blocked lanes. This was combined with a reduced speed area on the adjacent lanes to emulate drivers slowing down to observe the incident (Hadi et al., 2007; Zhou and Tian 2012). The length of "reduce speed area" in the vicinity lanes of the incident was modified by trial and error to achieve the abovementioned expected drop in capacity due to the uninterrupted incident. "Uninterrupted incident" refers to the incident that is not affected by downstream signal queue spillbacks.

\subsubsection{Assessing the Impacts of Downstream signal on Upstream Incident Capacity}

As discussed in the previous section, the first model developed in this study estimates the impact of queue spillbacks from downstream signalized intersections on the capacity of midblock incident locations. The modeled incidents are one- and two-lane blockage incidents on a three-lane urban street segment at different distances from the 
downstream intersection and different $\mathrm{g} / \mathrm{C}$ ratios at the downstream intersection. The model is applicable for conditions, in which the capacity with incident conditions is below the demand at the incident location.

The simulation started with a 15 -minute warm-up period, followed by a 75 -minute analyzing period. In all simulated scenarios, a one-hour incident was assumed to occur 15 minutes after the simulation starts. The simulation model was used to assess the impacts of incidents on upstream intersection throughputs. The incident location and the downstream signal $\mathrm{g} / \mathrm{C}$ ratio were varied, and the incident capacity at the upstream link was assessed using the microscopic simulation. Figure 6-2 shows the variation in the incident capacity with incident location and the $\mathrm{g} / \mathrm{C}$ ratio of downstream signalized intersection, considering the segment number of lane blockages.

As seen in Figure 6-2, the drop in the incident capacity decreases with the increase in the distance from the downstream intersection. In addition, the capacity increases with the increase in the $\mathrm{g} / \mathrm{C}$ ratio at the downstream intersection and decreases with the increase in the number of blocked lanes. 


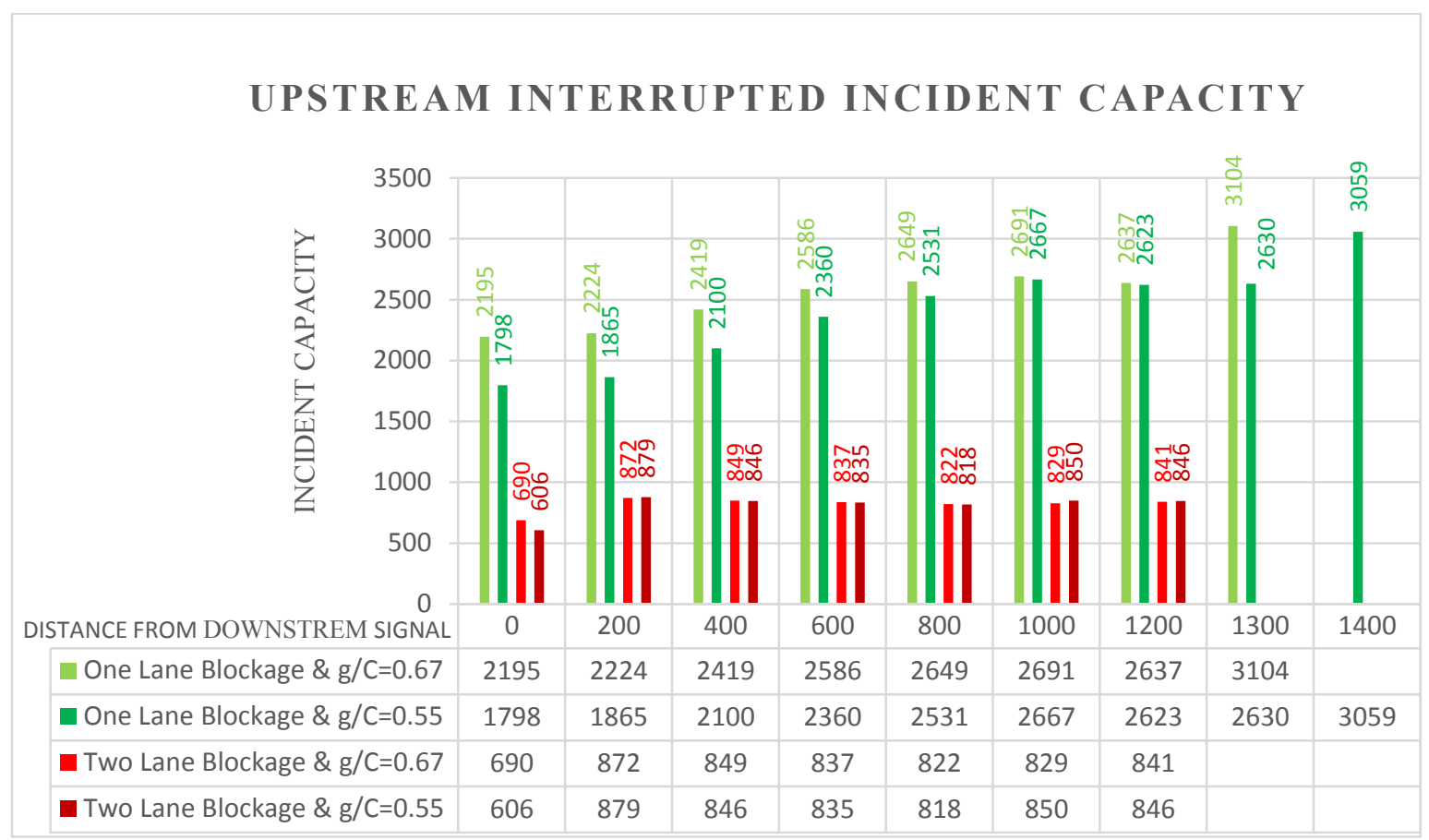

Figure 6-2 Interrupted Incident Capacity Effected by Downstream Intersection

Based on the simulation results for one lane blockage, the upstream incident capacity was not found to be affected by the downstream intersection when the incidents occur at 1,310 ft. and 1,420 ft. from the downstream signal and when the $\mathrm{g} / \mathrm{C}$ ratio at the downstream signal equals 0.67 and 0.55 , respectively. These values were selected for use in the analysis based on typical $\mathrm{g} / \mathrm{C}$ ratios for the main street in the analysis area. Reducing the $\mathrm{g} / \mathrm{C}$ ratio to a level that constrains the departing volumes from the upstream link is expected to reduce the traffic volume arriving at the downstream incident location, and this may reduce the portion of unconstrained green. For the two-lane blockage incident, the capacity at the incident location was not affected significantly by the downstream 
intersection when the incident occurs at $250 \mathrm{ft}$. or more from the downstream signal. This distance is the same for both $\mathrm{g} / \mathrm{C}$ ratios $(0.55$ and 0.67$)$.

The data presented in Figure 6-2 was used to develop regression models to estimate the incident capacity (IC) based on the investigated influencing factors. The developed regression models are presented in Table 6-1. The developed regression models show that there is a significant relationship between the incident capacity and the three independent variables, mentioned earlier, as indicated by the Coefficient of Determination (R-Squared) values and the t-test of the significance of the independent variable coefficients. If the $\mathrm{g} / \mathrm{C}$ ratio for an assessed condition is between the two $\mathrm{g} / \mathrm{C}$ ratios assessed in this study, as displayed in Table 6-1, interpolation can be used to estimate the capacity.

Table 6-1 Upstream Interrupted Incident Capacity Regression Models

\begin{tabular}{|c|c|c|c|}
\hline $\begin{array}{c}\text { Number of } \\
\text { Lane } \\
\text { Blockage }\end{array}$ & $\begin{array}{c}\text { Downstream } \\
\text { Signal g/C }\end{array}$ & Upstream Incident Capacity & $\mathbf{R}^{\mathbf{2}}$ \\
\hline $\begin{array}{c}\text { One Lane } \\
\text { Blockage }\end{array}$ & 0.67 & $\mathrm{IC}=0.7924 \mathrm{x}+1796.2$ & 0.9406 \\
\cline { 2 - 4 } & 0.55 & $\mathrm{IC}=0.6322 \mathrm{x}+2153.5$ & 0.9196 \\
\hline \multirow{2}{*}{$\begin{array}{c}\text { Two Lane } \\
\text { Blockage }\end{array}$} & 0.67 & $\mathrm{IC}=6 \mathrm{E}^{-07} \mathrm{x}^{3}-0.0016 \mathrm{x}^{2}+1.2092 \mathrm{x}+633.45$ & 0.9215 \\
\cline { 2 - 4 } & 0.55 & $\mathrm{IC}=4 \mathrm{E}^{-07} \mathrm{x}^{3}-0.0011 \mathrm{x}^{2}+0.839 \mathrm{x}+706.85$ & 0.8982 \\
\hline
\end{tabular}

\subsubsection{Assessing the Impacts of Incidents on Upstream Intersection Maximum Throughputs}

A second model was developed in this study to estimate upstream intersection maximum throughput as impacted by the drop in capacity due to a downstream signal. The maximum throughput was assessed for incidents with different distances from the upstream intersection, different volume-to-capacity incident link ratios (v/Ic), and different incident 
durations. This was conducted by introducing incidents at different locations with different capacity drops and different incident durations in a VISSIM model. The incident durations were 15 minutes, 35 minutes, and 45 minutes. It should be noted that 35 minutes is the median incident duration at the study location. The network used in the testing is part of the Glades Road network in Boca Raton, Florida.

First, the network was simulated without an incident, and then incidents with different attributes were introduced in the model. In all simulated incident scenarios, the incidents were assumed to occur 15 minutes after start of the simulation. The simulation model was used to assess the impacts of incidents on upstream intersection maximum throughputs. The maximum throughputs at the upstream intersection were assessed using the microscopic simulation.

Figures 6-3 to 6-5 show the variation in the upstream intersection maximum throughput with the incident location, incident duration and the v/Ic ratio at the incident location. In these figures, the incident location references the distance from the upstream signal stop line. It should be also noted that the saturation flow of the upstream signal without incident is $5,562 \mathrm{veh} / \mathrm{hr}$. Thus, incidents with downstream locations and v/Ic ratios that produce this upstream intersection saturation flow in the simulation are recognized as incidents that do not impact upstream signal operations. For example, the investigated 35minute incident did not affect the upstream intersection throughput when it is located more than $1550 \mathrm{ft}$., $2400 \mathrm{ft}$., and $3000 \mathrm{ft}$. from the upstream signalized intersection with the v/Ic ratio at the incident location of $1.13,1.3$ and 1.5 , respectively. 


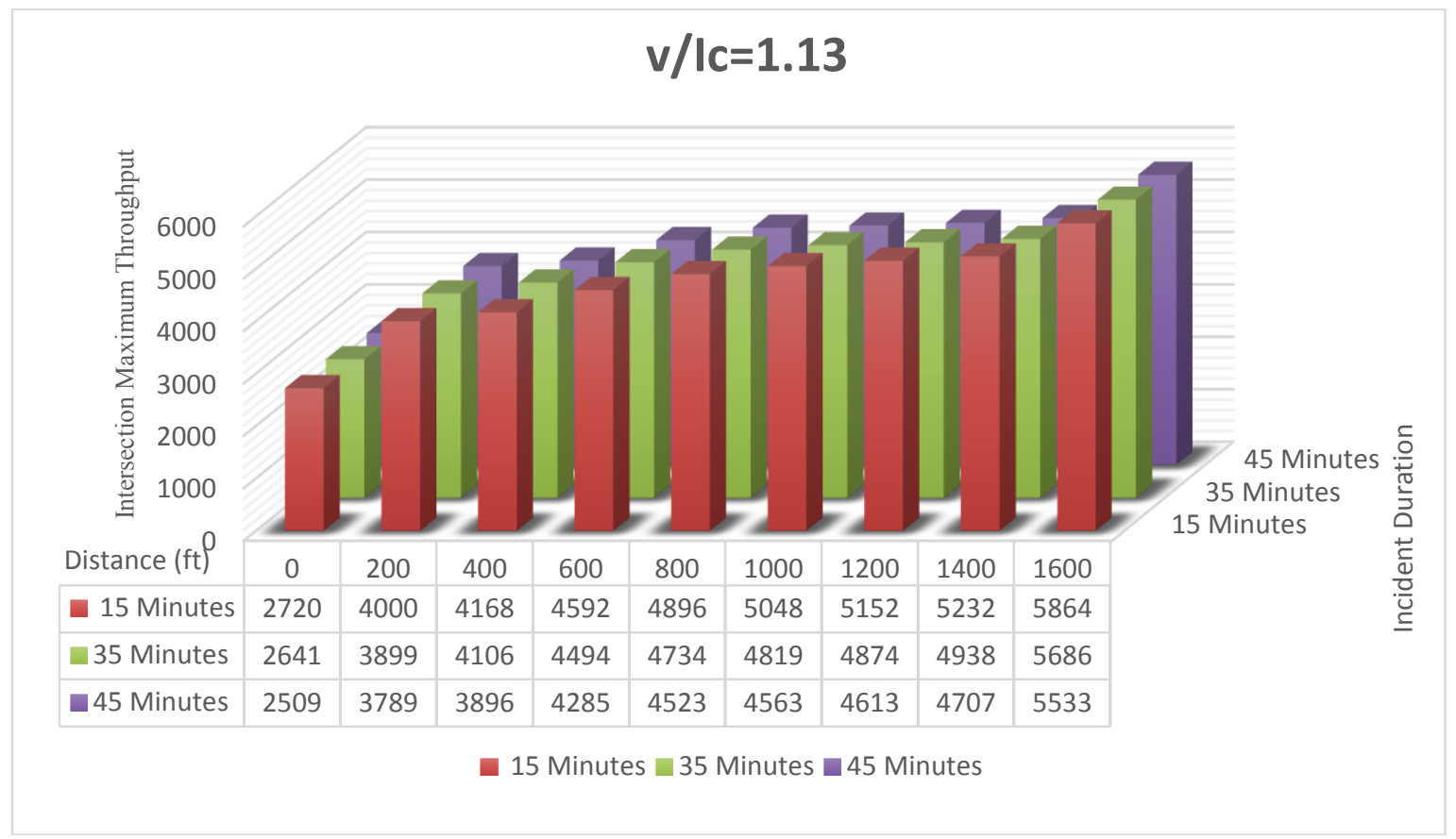

Figure 6-3 Upstream Intersection Maximum Throughput Variation with Incident Location and Duration $(\mathrm{v} / \mathrm{Ic}=1.13)$ at the Incident Location

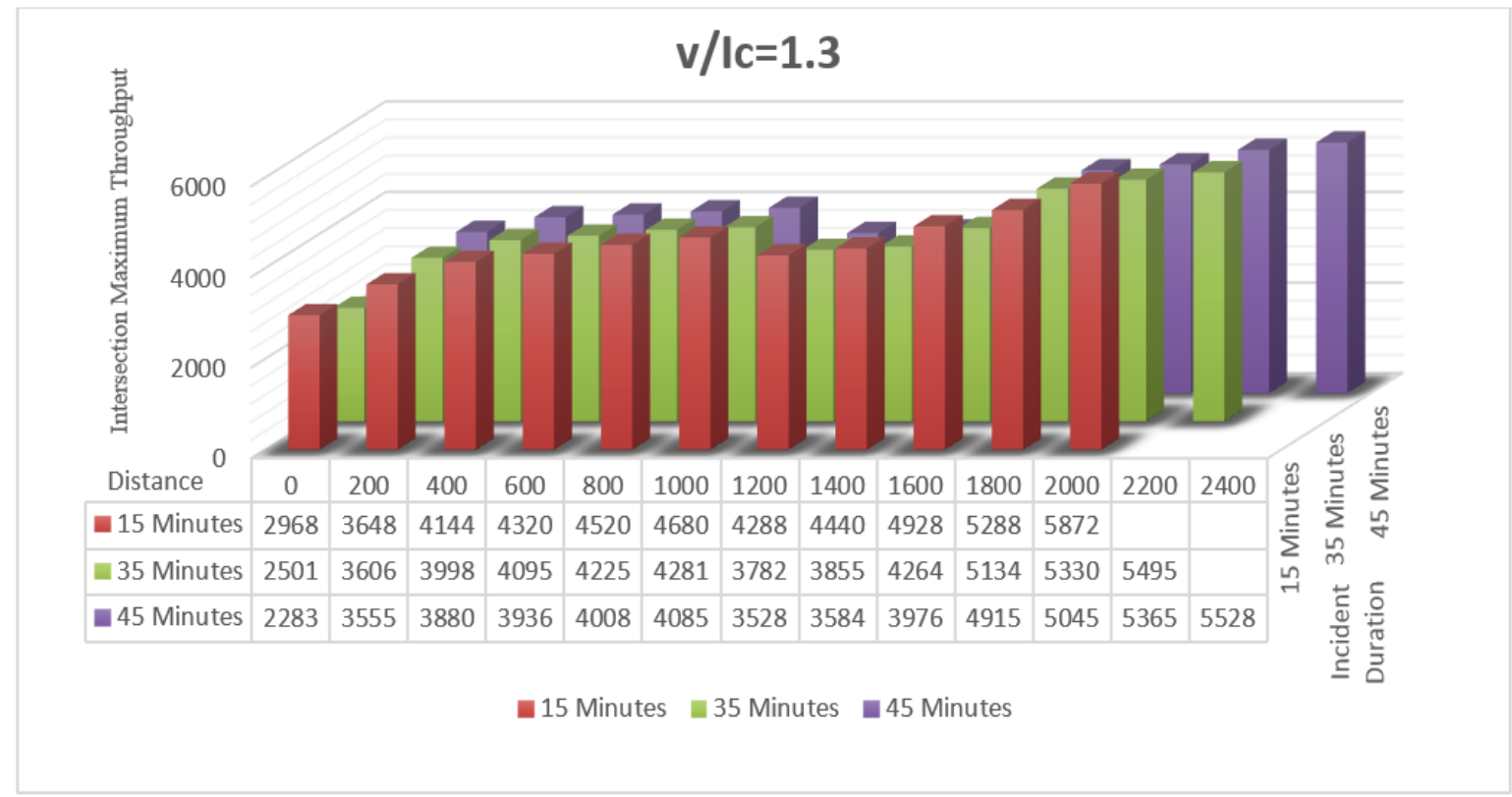

Figure 6-4 Upstream Intersection Maximum Throughput Variation with Incident Location and Duration $(\mathrm{v} / \mathrm{Ic}=1.3)$ at the Incident Location 


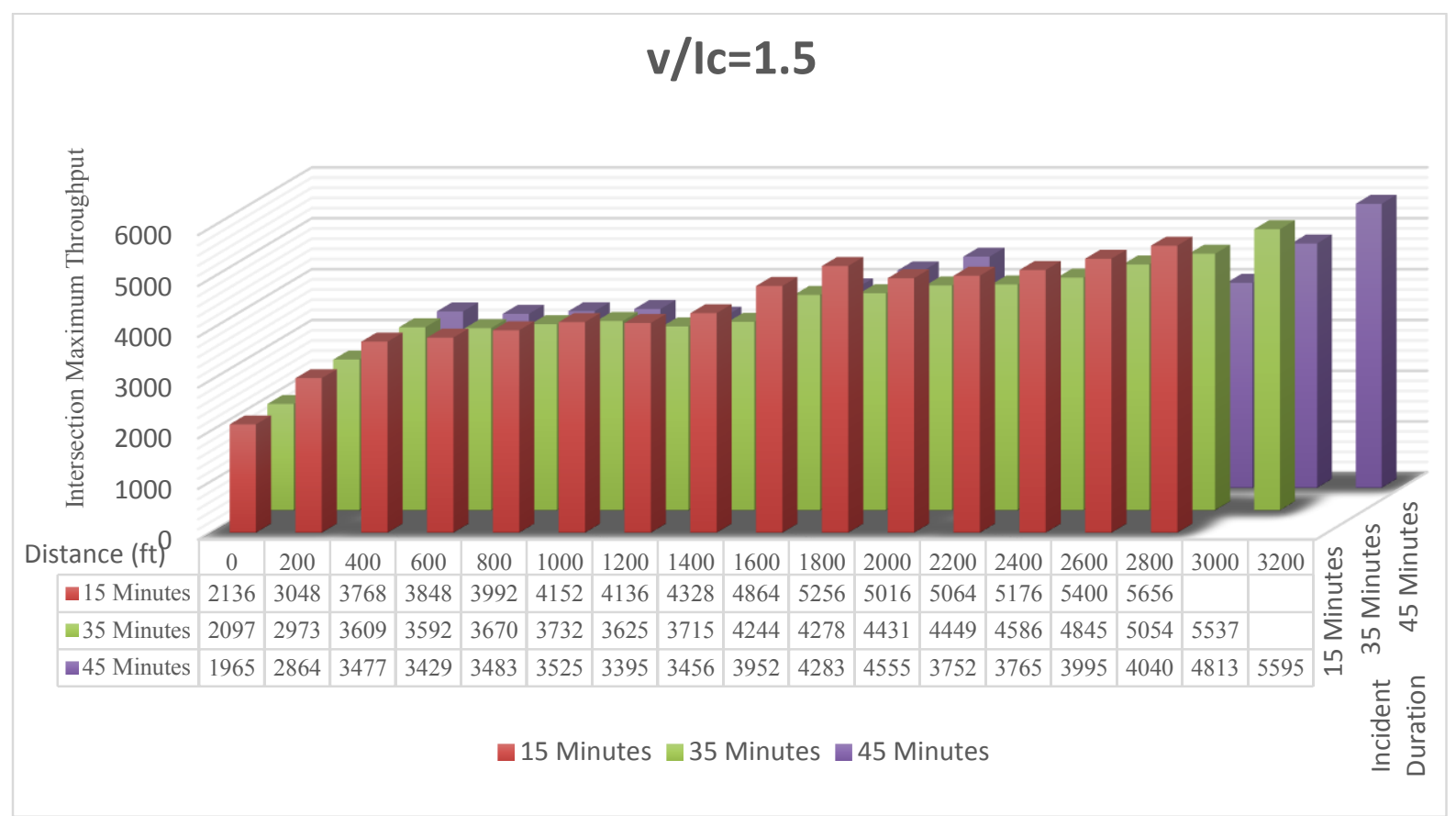

Figure 6-5 Upstream Intersection Maximum Throughput Variation with Incident Location and Duration $(\mathrm{v} / \mathrm{Ic}=1.5)$ at the Incident Location

As seen in Figures 6-3 to 6-5, the throughput decreases with the increase in the $\mathrm{v} / \mathrm{Ic}$ ratio at the incident location and the decrease in the distance between the upstream intersection and the incident location. The incident duration effect on the throughput can be explained by the fact that in the first few minutes after the incident occurs, the queue length does not always reach the upstream signals, and thus the upstream intersection operates close to the saturation flow rate during the first few minutes. Since the proportions of these minutes compared to the incident duration are higher in the case of shorter incidents, the calculated throughput of shorter incidents are somewhat higher.

As stated earlier, when an incident occurs at a location downstream of an upstream intersection, the throughput of the upstream links that feed the incident link can decrease 
if the queue from the incident spills back to the upstream links. For incidents that cause queueing due to demand exceeding the capacity at the incident location, when the signal phases serving the upstream feeding links are red, the downstream link queue starts decreasing due to the reduction in the arrivals at the back of the queue. This creates some queuing capacity that can accommodate flows from the upstream links in the green phase. During the first parts of the upstream link green phases, referred to as the "unconstrained green", the vehicles will be able to leave the stop lines of the feeding links at the saturation flow rates of these links until the queue due to the downstream incident spills back to the upstream signal again. The duration of the unconstrained green was calculated based on the following relationships:

$$
M T=S F \times \frac{U G}{C}+I c \times \frac{(T G-U G)}{C}
$$

Thus, the unconstrained green can be calculated as:

$$
U G=\frac{(M T \times c-I c \times T G)}{(S F-I c)}
$$

where

$$
\begin{aligned}
& U G=\text { upstream intersection unconstrained green time, } \\
& M T=\text { intersection maximum throughput, } \\
& T G=\text { upstream intersection green time, } \\
& C=\text { cycle length, } \\
& I c=\text { capacity at the incident location, and } \\
& S F=\text { saturation flow. }
\end{aligned}
$$


Table 6-2 Variation in Upstream Unconstrained Green in Terms of Incident location, Duration and Volume to Incident Capacity (v/Ic) Ratio

\begin{tabular}{|c|c|c|c|c|}
\hline $\begin{array}{l}v / \text { Ic at } \\
\text { incident } \\
\text { location }\end{array}$ & $\begin{array}{l}\text { Incident } \\
\text { Location }\end{array}$ & $\begin{array}{l}\text { Unconstrained } \\
\text { Green for } 15 \\
\text { Minute Incident }\end{array}$ & $\begin{array}{l}\text { Unconstrained } \\
\text { Green for } 35 \\
\text { Minute Incident }\end{array}$ & $\begin{array}{l}\text { Unconstrained } \\
\text { Green for } 45 \\
\text { Minute Incident }\end{array}$ \\
\hline \multirow{8}{*}{1.13} & 200 & 29.05 & 25.43 & 21.50 \\
\hline & 400 & 35.07 & 38.65 & 25.32 \\
\hline & 600 & 50.25 & 50.33 & 39.27 \\
\hline & 800 & 61.14 & 55.34 & 47.77 \\
\hline & 1000 & 66.59 & 58.39 & 49.20 \\
\hline & 1200 & 70.31 & 60.36 & 51.02 \\
\hline & 1400 & 73.18 & 62.65 & 54.36 \\
\hline & 1600 & 80 & 80 & 80 \\
\hline \multirow{12}{*}{1.3} & 200 & 26.69 & 25.41 & 23.84 \\
\hline & 400 & 41.80 & 37.35 & 33.76 \\
\hline & 600 & 47.16 & 40.31 & 35.46 \\
\hline & 800 & 53.25 & 44.27 & 37.66 \\
\hline & 1000 & 58.13 & 45.97 & 40.01 \\
\hline & 1200 & 46.19 & 30.77 & 23.03 \\
\hline & 1400 & 50.82 & 32.99 & 24.74 \\
\hline & 1600 & 65.68 & 45.46 & 36.68 \\
\hline & 1800 & 76.65 & 71.96 & 65.28 \\
\hline & 2000 & 80.00 & 77.93 & 69.26 \\
\hline & 2200 & 80.00 & 80.00 & 79.01 \\
\hline & 2400 & 80.00 & 80.00 & 80.00 \\
\hline \multirow{16}{*}{1.5} & 200 & 17.38 & 15.36 & 12.43 \\
\hline & 400 & 36.74 & 32.47 & 28.93 \\
\hline & 600 & 38.90 & 32.01 & 27.63 \\
\hline & 800 & 42.77 & 34.11 & 29.07 \\
\hline & 1000 & 47.07 & 35.78 & 30.22 \\
\hline & 1200 & 46.64 & 32.90 & 26.70 \\
\hline & 1400 & 51.81 & 35.32 & 28.35 \\
\hline & 1600 & 66.22 & 49.55 & 41.69 \\
\hline & 1800 & 76.77 & 50.46 & 50.59 \\
\hline & 2000 & 70.31 & 54.58 & 57.90 \\
\hline & 2200 & 71.60 & 55.06 & 36.31 \\
\hline & 2400 & 74.62 & 58.75 & 36.67 \\
\hline & 2600 & 78.00 & 65.71 & 42.84 \\
\hline & 2800 & 80.00 & 71.34 & 44.06 \\
\hline & 3000 & 80.00 & 80.00 & 64.86 \\
\hline & 3200 & 80.00 & 80.00 & 80.00 \\
\hline
\end{tabular}


Table 6-2 presents the variation in the unconstrained green as a function of the demand to capacity ratio at the incident location, incident duration and the distance from the upstream intersection to the incident location. Table 6-3 shows the regression analysis models developed in this study to estimate the maximum throughput and the unconstrained green of the upstream intersection as a function of the independent variables. The $\mathrm{x}$ in the table is the incident distance from the upstream intersection in feet.

Table 6-3 Regression Models to Estimate Upstream Intersection Maximum Throughput and Unconstrained Green Regression Models

\begin{tabular}{|c|c|c|c|c|c|}
\hline $\begin{array}{c}\text { Incident } \\
\text { Duration }\end{array}$ & $\begin{array}{c}\text { v/Ic at } \\
\text { Incident } \\
\text { Location }\end{array}$ & $\begin{array}{c}\text { Intersection } \\
\text { Movement Maximum } \\
\text { Throughput }\end{array}$ & $\mathbf{R}^{\mathbf{2}}$ & Unconstrained Green & $\mathbf{R}^{\mathbf{2}}$ \\
\hline \multirow{3}{*}{$\begin{array}{c}15 \\
\text { Minutes }\end{array}$} & 1.13 & $\mathrm{MT}=0.4858 \mathrm{x}+1143.6$ & 0.84 & $\mathrm{UG}=0.0449 \mathrm{x}+15.785$ & 0.9 \\
\cline { 2 - 6 } & 1.3 & $\mathrm{MT}=0.3317 \mathrm{x}+1146.7$ & 0.83 & $\mathrm{UG}=0.0305 \mathrm{x}+19.136$ & 0.80 \\
\cline { 2 - 6 } & 1.5 & $\mathrm{MT}=0.3334 \mathrm{x}+994.31$ & 0.88 & $\mathrm{UG}=0.0251 \mathrm{x}+18.117$ & 0.88 \\
\hline \multirow{3}{*}{$\begin{array}{c}35 \\
\text { Minutes }\end{array}$} & 1.13 & $\mathrm{MT}=0.4628 \mathrm{x}+1113.7$ & 0.83 & $\mathrm{UG}=0.0403 \mathrm{x}+15.696$ & 0.87 \\
\cline { 2 - 6 } & 1.3 & $\mathrm{MT}=0.3242 \mathrm{x}+1048$ & 0.72 & $\mathrm{UG}=0.0275 \mathrm{x}+14.143$ & 0.73 \\
\hline \multirow{3}{*}{45 Minutes } & 1.5 & $\mathrm{MT}=0.2761 \mathrm{x}+928.35$ & 0.89 & $\mathrm{UG}=0.0233 \mathrm{x}+17.439$ & 0.80 \\
\cline { 2 - 6 } & 1.13 & $\mathrm{MT}=0.4632 \mathrm{x}+1053.4$ & 0.82 & $\mathrm{UG}=0.04 \mathrm{x}+8.9462$ & 0.90 \\
\cline { 2 - 6 } & 1.3 & $\mathrm{MT}=0.3256 \mathrm{x}+985.88$ & 0.73 & $\mathrm{UG}=0.0266 \mathrm{x}+10.23$ & 0.73 \\
\hline
\end{tabular}

\subsection{Estimation of Incident Diversion Rate}

To estimate the incident diversion, this research developed a logit regression model that estimates the diversion rate due to incidents on urban streets. Implementing this model in conjunction with the mesoscopic simulation-based DTA tool (DTAlite) provides the 
vehicle diversion at the incident location and diversion routes. . The logit model estimates the percentage of the potential drivers for an incident with given attributes, and the DTA model identifies the diversion routes.

Diversion rates due to incidents are expected to be different for different systems depending on traffic conditions at the original and alternative routes. It also depends on incident attributes such as the incident duration, number of lanes blockage, and location of the incident. The data used in this study to estimate the logit parameters include archived traffic detector and incident databases. Traffic management centers in Florida maintain detailed incident management archives in Oracle database files. The incident archives include incident time stamps (detection, notification, responses, arrivals, and departures), event details (severity, number of blockage lane, duration), and incident locations. The traffic database contains traffic volume, speed, and occupancy measurements at different aggregation levels (15-minute aggregation level used in this study). To estimate the diversion, incidents on two corridors (Broward Boulevard and Sunrise Boulevard from I95 to US-1 in Broward County, Florida) were selected in the analysis. A set of incidents and associated attributes during evening peak hours (3-7 pm), between May 1, 2014 and October 30, 2016, were extracted from the incident database. The selected incidents were then associated with measurements from traffic detector stations at locations upstream and downstream of the incident locations. This association provides the determination of the diversion rate at each detector location based on the detector measurements. The incident diversion rate was estimated based on the average difference between the median traffic 
volumes of typical no-incident days and the volumes for the incident days. Therefore, the average typical median no-incident day and incident day traffic volumes needs to be estimated before the diversion rate can be calculated.

\subsubsection{Traffic Volume Estimation for No Incident Days}

The demand level with no-incident condition was selected as the median demand during weekdays without incidents or abnormal external conditions. The days with incidents, special events, and bad weather were first filtered out. In addition, holidays and weekends were removed from the analysis. The remaining days were included in the next step analysis. The 15-minute traffic counts between 3:00 pm and 7:00 pm for the remaining days were aggregated to obtain the PM peak period traffic counts. Figures 6-6 and 6-7 show the volumes for Broward and Sunrise Boulevards during the PM peak period for normal days between June 1, 2015 and May 31, 2016. The different percentile volumes of the normal days during the PM peak period were calculated and are shown in Figures 6-6 and 6-7 as straight lines. The days with volumes that are close to the $50^{\text {th }}$ percentile values were considered representative days for these two corridors and used in subsequent analysis. It should be noted that there may be no days with exact volumes as the $50^{\text {th }}$ percentile. In this case, the average volume of the two closest days was used in the analysis. 


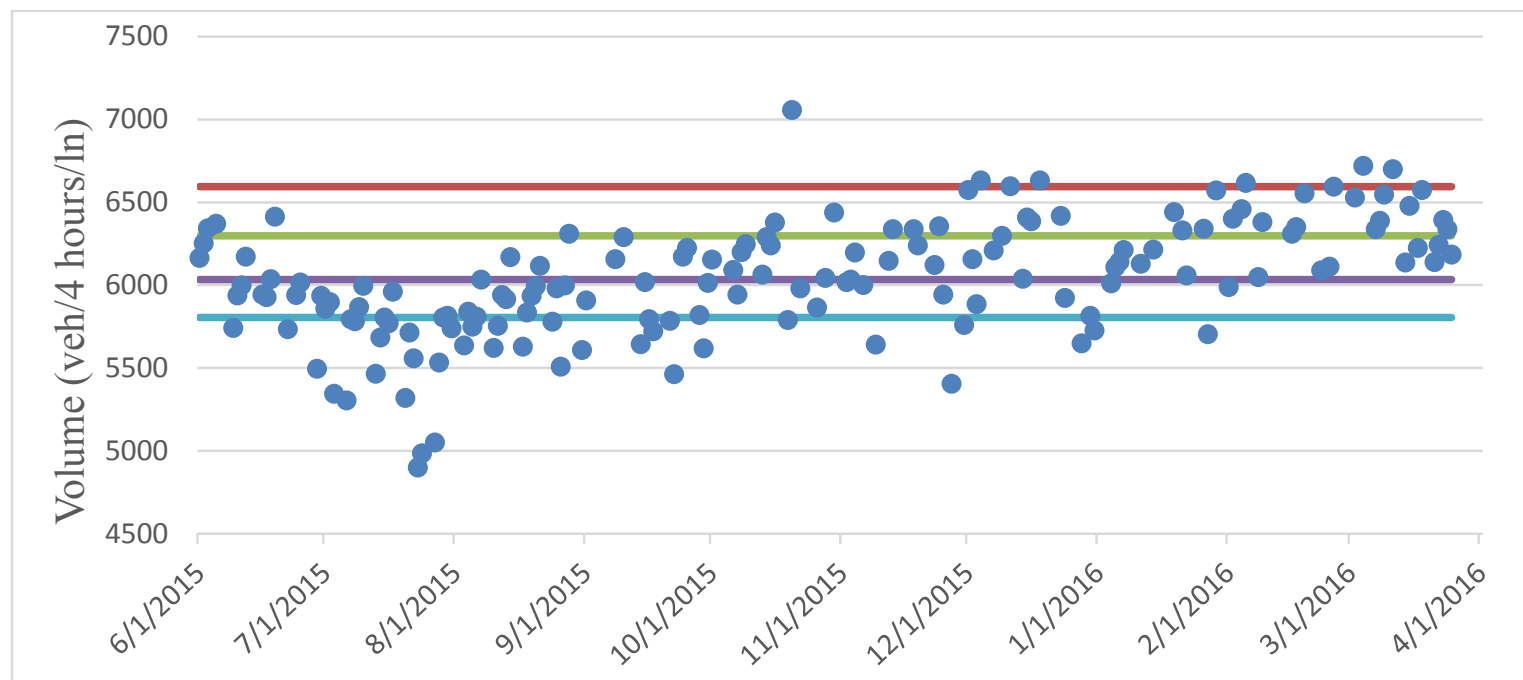

Normal Reperesentative Study Days from June 2015 untill April 2016

$\longrightarrow 95$ Percentile $\longrightarrow 75$ Percentile $\longrightarrow 50$ Percentile $\longrightarrow 25$ Percentile $\quad$ Average Volume per Lane

\section{Figure 6-6 Broward Blvd Eastbound Volume Distribution (PM Peak)}

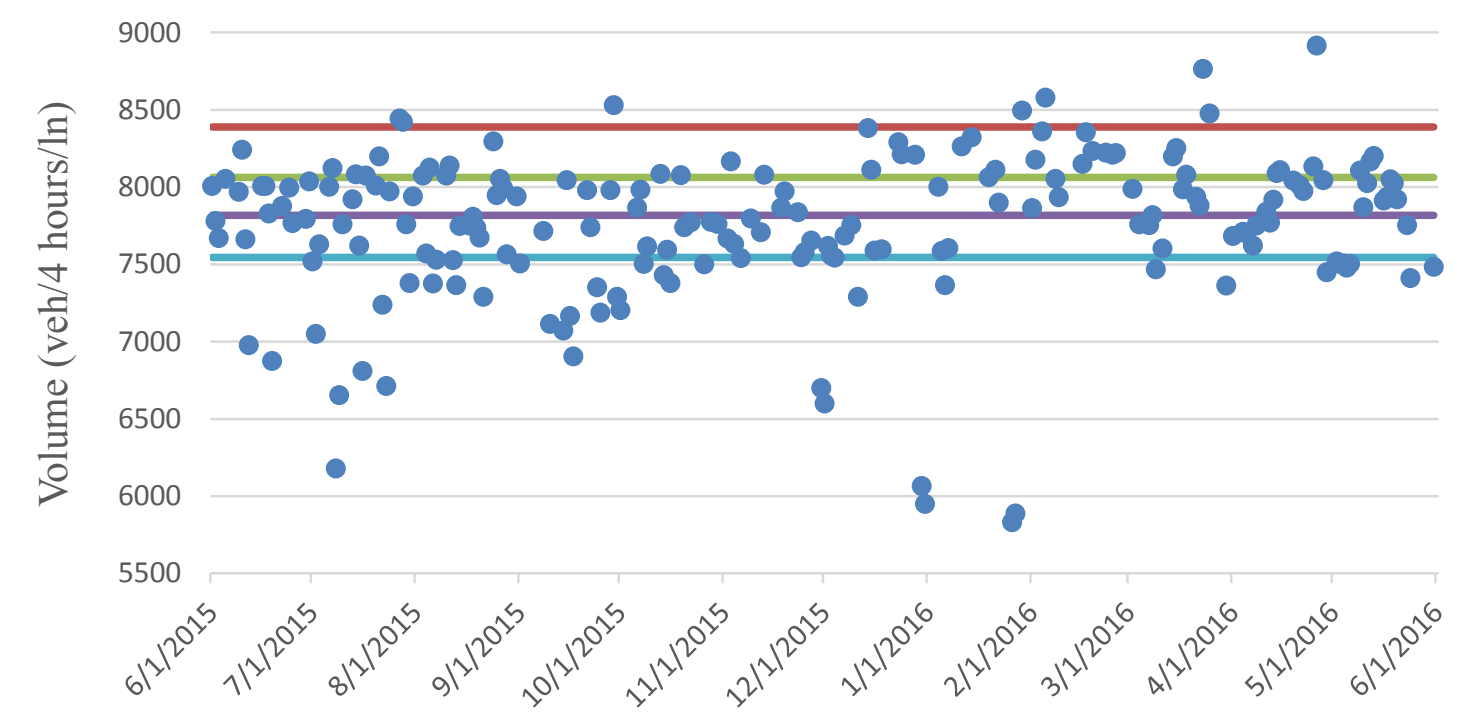

Normal Reperesentative Study Days from June 2015 untill June 2016

$\longrightarrow 95$ Percentile $\longrightarrow 75$ Percentile $\longrightarrow 50$ Percentile $\longrightarrow 25$ Percentile $\bullet \quad$ Average Volume per Lane

Figure 6-7 Sunrise Blvd Eastbound Volume Distribution (PM Peak) 


\subsubsection{Diversion Rate Estimation}

The estimation of the diversion rate requires the estimation of the volumes during each incident for use in the analysis and for comparison with the estimated median day's volumes, as described in the previous section. The volumes for the day incidents during PM peak hours were also extracted from database. This requires the association of the time stamps and the location of the incident with traffic detector data.

Thirty five one-lane blockage incidents during the PM peak period were identified, and time-varying counts for each incident were captured. Figure 6-8 shows the incident and normal median days' counts on the Sunrise and Broward Boulevards. To estimate the diversion rates for each incident, the difference between the normal traffic day and the incident day over a period from the occurrence of the incident to the end of the recovery time was estimated. Thus, the analysis period includes the incident duration and incident recovery period. The incident recovery period starts once the incident is cleared until the incident queue dissipates. Let $V_{i N}$ denote the volume of detectors for time interval $i$ during the normal traffic day conditions, and $V_{i I}$ denote the volume for the time interval $i$ during a specific incident day condition. The $t$ is the incident time occurrence and $\Delta t$ is the mathematical expression used to calculate the diversion rate in percentage due to the incident $(D)$, as follows:

$$
\mathrm{D}=\frac{\int_{\mathrm{i}=\mathrm{t}}^{\mathrm{t}+\Delta \mathrm{t}} V_{i N^{-}} \int_{\mathrm{i}=\mathrm{t}}^{\mathrm{t}+\Delta \mathrm{t}} V_{i I}}{\int_{\mathrm{i}=\mathrm{t}}^{\mathrm{t}+\Delta \mathrm{t}} V_{i N}} \times 100
$$




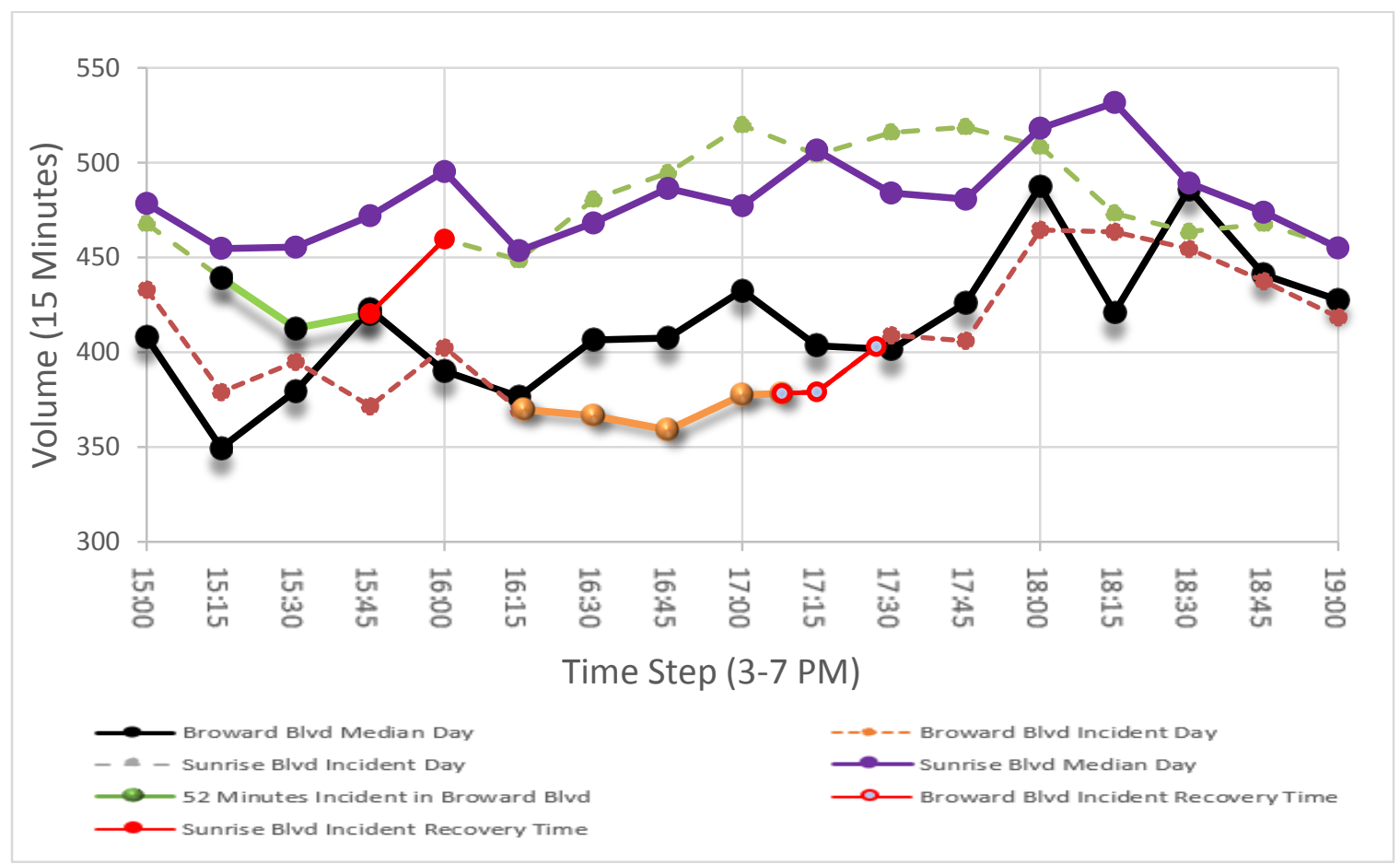

Figure 6-8 Two Examples Of Broward \& Sunrise Normal and Incident Day Counts Comprision

One of the challenges of diversion rate estimation is to identify the analysis period for which the cumulative volumes with and without incidents are compared to estimate the diversion. The analysis period in this research is set to the time that the incident starts until the time that the queue caused by the incident dissipates. The associated records in the incident database only report the time stamp at which the lane blockage ends, and there is no information about the time stamp when the traffic returns to normal conditions (recovery time stamp). To identify the incident recovery time, the volumes in the incident day and median normal days were plotted during the PM peak period. Two curves were compared, and the end of the recovery time was identified when the volumes in the two curves were close to each other. The normal day pattern and incident day pattern in terms of the traffic 
volume were compared to determine where the traffic volume appears to start to be affected by the incident and when this effect appears to end. The difference in the volume summation between these two points was obtained and assumed to be equal to the diverted volume. As shown in Figure 6-8, the study corridor normal median and incident day counts at 15-minutes interval were plotted for the purpose of diversion estimation. The incident started at 16:16 PM and it cleared after 52 minutes at 17:08 PM, but the queue did not dissipate at the time shown 17:27 PM. Another example of incident occurrence at Sunrise Boulevard indicates that incident starts at 3:15 PM and it was removed after 30 minutes, whereas the count data shows that the queue dissipated after 15 minutes of incident clearance.

\subsubsection{Diversion Model}

All lane blockage incidents that occurred on the Broward and Sunrise Boulevards between 3:00 p.m. and 7:00 p.m. on weekdays, from May 1st, 2014 to October 30th, 2016, were identified for use in the regression model. Since there was no sufficient incident database for 2 and 3-lane blockages, only the incidents with a one-lane blockage were used in the regression model. Figure 6-9 shows the general relationship between the incident duration and diversion rate. As expected, the diversion rate increased as the incident duration increased. However, a significant diversion for the incidents that have a duration less than 15 minutes was not found. Therefore, the derived model can be used for the incidents that have a duration between 15 and 90 minutes. The derived expression using the regression analysis was as follows: 


$$
D=7.1148 \ln (I D)-17.879
$$

where

$\mathrm{D}=$ average diversion rate in percentage, and

ID $=$ incident duration in minutes

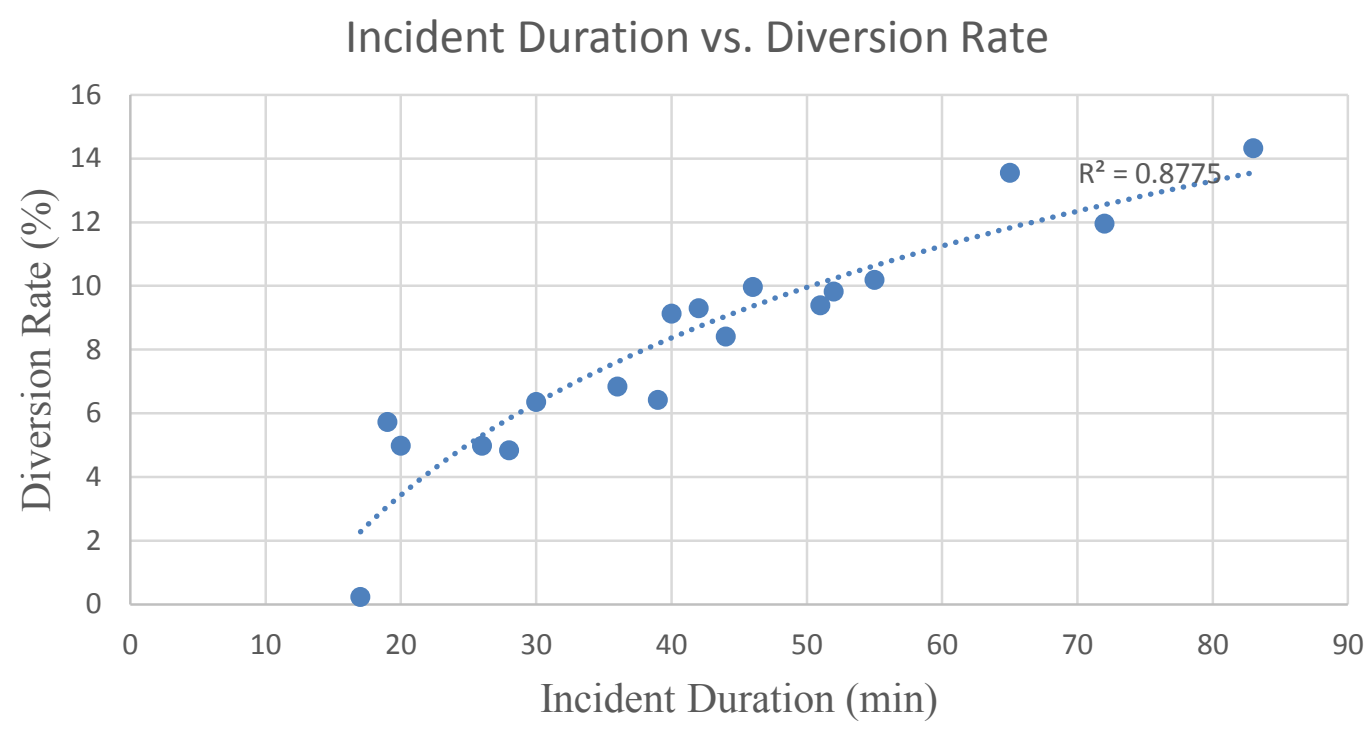

Figure 6-9 Relationship Between Average Diversion Rate and Incident Duration

As seen in Figure 6-9, the model fits the data relatively well with a $\mathrm{R}^{2}$ of 0.88 . This expression indicates that there is a general trend of increase in diversion with the increase in the incident duration.

\subsection{Modeling Incidents in Mesoscopic Simulation}

The regression model, derived as discussed in Section 6.3, was implemented in conjunction with the mesoscopic simulation-based DTA tool. The logit model was used to estimate the diversion rate and the DTA tool was used to estimate the alternative paths that 
vehicles will take utilizing the dynamic traffic assignment (DTA). Therefore, it is necessary to model the incident in the DTA tool. The utilized tool (DTAlite) cannot account for the location of the incident in the midblock and can only model the incident at the stop line. In addition, as described later in this section, it may not be able to model the additional impacts due to the inefficiency associated with queue spillbacks at the upstream and downstream intersections. Thus, the models derived in Section 6.2, were used to supplement the incident modeling in the tool. In order to model the incident, first the capacity at the incident location was estimated using the models presented in Table 6-1. This capacity can be used as an input to the models presented in Table 6-3 to determine the impact of the midblock incident on upstream intersection maximum throughput. The results were compared with the impact of the incident on the upstream signal operation as assessed by DTAlite to determine, if additional fine-tuning of the upstream signal operation is needed to reflect the impacts of the midblock incidents considering their locations. This is done to account for the inefficiency resulting from vehicles of a main-street through movement that feeds the downstream link getting the green time but is not be able to move due to the downstream link being temporary blocked. The cross streets may fill the queueing storage that becomes available during the rest of the main street through movement. The model presented in Table 6-1 considers theses inefficiencies.

This approach was tested by running DTAlite with a small hypothetical network that has five nodes and four links. The first upstream link was designed to accommodate the expected queue of the arriving vehicles to the network, in case of spillback from the 
incident. The third link was considered as the link, on which an incident occurs at the midblock. The second link was the incident upstream link. One lane blockage incident with a 35-minute duration was modeled $600 \mathrm{ft}$. downstream from the upstream signal and $800 \mathrm{ft}$. away from the downstream signal. The incident started in the 990 -minute time step of the simulation and was removed in the 1,025-minute time step. Five scenarios were developed to analyse the performance of the incident modeling in different ways in the DTAlite. The first scenario was implemented to model the base condition without the incident. In the second scenario, the incident was modeled with the drop in the incident link capacity without considering the incident location, as is done in DTAlite models. Since the DTAlite is capable of modeling the incident at the intersection stop line only, a third scenario was designed by defining a dummy link at the incident location to emulate the incident location impact on the spillback. It should be noted that in the second and third scenarios, the drop in the capacity at the incident location was calculated based on the SHRP2 Program L08 project procedure of calculating the capacity drops due to the incidents on arterial facilities (Zegeer et al., 2014). The last scenario was implemented by using the proposed method that combines the derived regression model in Section 6.2 with the DTA tool modeling. When applying the regression model in Table 6-4 to the case study, the following values were set: Ic is the capacity during incident ( $2096 \mathrm{veh} / \mathrm{hr}$ ), $\mathrm{n}=3$ is number of lanes of the incident link, and $\mathrm{C}$ is the lane capacity with no incident $(800$ $\mathrm{veh} / \mathrm{hr} / \mathrm{ln})$. The lane capacity was calculated based on the link saturation flow rate of 1600 $(\mathrm{veh} / \mathrm{hr} / \mathrm{ln})$ to an effective green per cycle length ratio, which was 0.50 . The noted 
downstream $\mathrm{g} / \mathrm{C}$ ratio is 0.55 . The upstream signal $\mathrm{g} / \mathrm{C}$ ratio is also 0.60 , so that, the upstream node maximum flow is $2880(\mathrm{veh} / \mathrm{hr})$ for three lanes. The V/Ic ratio at the incident location is equal to 1.40. By using the regression model in Table 6-2, the upstream signal maximum saturation flow was calculated $(950 \mathrm{veh} / \mathrm{hr} / \mathrm{ln}$ or $2850 \mathrm{veh} / \mathrm{hr})$. Thus, the upstream signalized intersection capacity during the incident can be calculated by multiplying the upstream signal maximum throughput (2850 veh/hr) by its $\mathrm{g} / \mathrm{C}$ ratio (0.6), which is equal to $1710(\mathrm{veh} / \mathrm{hr})$ or $(427 \mathrm{veh} / 15 \mathrm{~min})$.

As seen in the Table 6-4, the upstream signal capacity is about 690 (veh/15 minutes) without the incident. The second scenario shows that the upstream signal capacity starts to drop once the queue from the incident capacity location reaches the upstream signal according to the mesoscopic simulation modeling associated with the DTA tool. The results show that the queue reaches the upstream node 15 minutes after the incident occurred because the utilized mesoscopic models the incident at the stop line. Also, the results show that the percentage drop in the incident capacity is the same at the incident location and upstream intersection location once the shockwave reaches the upstream intersection, which does not account for the inefficiency at the upstream intersection location described earlier. In the third scenario, the incident location was modeled with a dummy link and without the downstream signal queue spillback effects on its downstream incident. As can be seen, although the drop in capacity occurred earlier, the same drop in capacity result in Scenario 3 as in Scenario 2. In the fourth scenario, the drop in the incident capacity was modeled based on dropping the capacity on the dummy link and accounting from 
downstream signal queue spillback. The downstream signal's effects on capacity is indicated by dropping the incident throughput from 500 (veh/15 min) to (474 veh/15 min). However, this drop still did not account for the inefficiency in upstream signal operations. The last scenario was designed based on the proposed method and the results show that the combination of the derived regression model and the DTA tool modeling can account for this effect by dropping the capacity further to 427 (veh/15 min).

Table 6-4 Upstream Signal Maximum Throughput for the Test Scenarios

\begin{tabular}{|c|c|c|c|c|c|c|}
\hline \multirow[b]{2}{*}{$\begin{array}{l}\text { Time } \\
\text { Stamp } \\
(\text { min) }\end{array}$} & Scenario 1 & Scenario 2 & Scenario 3 & \multicolumn{2}{|c|}{ Scenario 4} & Scenario 5 \\
\hline & $\begin{array}{l}\text { Upstream } \\
\text { Node } \\
\text { Throughput } \\
\text { (veh/15min) }\end{array}$ & $\begin{array}{c}\text { Upstream } \\
\text { Node } \\
\text { Throughput } \\
\text { (veh/15min) }\end{array}$ & $\begin{array}{c}\text { Upstream } \\
\text { Node } \\
\text { Throughput } \\
\text { (veh/15 min) }\end{array}$ & $\begin{array}{l}\text { Upstream } \\
\text { Node } \\
\text { Throughput } \\
\text { (veh/15min) }\end{array}$ & $\begin{array}{c}\text { Incident } \\
\text { Location } \\
\text { Throughput } \\
\text { (veh/15min) }\end{array}$ & $\begin{array}{l}\text { Upstream } \\
\text { Node } \\
\text { Throughput } \\
\text { (veh/15min) }\end{array}$ \\
\hline 975 & 158 & 158 & 158 & 158 & 158 & 158 \\
\hline 990 & 688 & 688 & 688 & 688 & 688 & 688 \\
\hline 1005 & 689 & 647 & 500 & 474 & 474 & 427 \\
\hline 1020 & 688 & 500 & 500 & 474 & 474 & 427 \\
\hline 1035 & 690 & 500 & 768 & 760 & 746 & 760 \\
\hline 1050 & 689 & 689 & 877 & 878 & 877 & 878 \\
\hline 1065 & 688 & 688 & 878 & 877 & 878 & 877 \\
\hline 1080 & 689 & 689 & 695 & 696 & 688 & 696 \\
\hline 1095 & 687 & 687 & 675 & 675 & 675 & 675 \\
\hline 1110 & 688 & 688 & 676 & 676 & 675 & 676 \\
\hline 1125 & 689 & 689 & 642 & 674 & 675 & 674 \\
\hline
\end{tabular}

\subsection{Modeling of Diversion in the DTA Tool}

To supplement the DTA-based analysis, the developed diversion regression model, described in Section 6.3, was combined with the traveler information modeling feature of the DTA tool to estimate the volumes at the incident location and the alternative routes. The utilized DTA features requires the users to input the percentages of drivers receiving Pre-trip and En-route information. The percentage of diverted drivers can be changed by 
varying the parameters associated with the modeling service. However, the examination of this feature in this study, showed that these parameters cannot be changed to affect the diversion in this version of the utilized DTA tool. It was determined that the percentage of diverted travelers could be changed by changing the number of travelers receiving the information. For example, for Broward Boulevard, it was determined that coding $25 \%$ of travelers receiving information resulted in a $6 \%$ diversion while the coding of $30 \%$ of travelers receiving information, resulted in a $9 \%$ diversion.

Three incident scenarios with different durations $(45,30$, and 15 minutes) were defined and coded in DTAlite for the Broward Boulevard Eastbound (EB) direction. All three incidents were assumed to occur $1000 \mathrm{ft}$ away from the upstream intersection $\left(3^{\text {rd }}\right.$ Ave and Broward Blvd) and $400 \mathrm{ft}$ away from the downstream intersection (US1 and Broward Blvd), starting 30 minutes after the simulation begins. As stated earlier, the regression models developed in Section 6-2 estimates the incident upstream links' maximum saturation flow rate and these links' capacities. Reducing links' capacities during the mentioned incidents were modified by trial and error, in order to achieve the expected drop in capacity due to the incident conditions shown in Figure 6-10. The volumes produced from the DTA models for the three case study incident scenarios, were imported to the microscopic simulation tool (VISSIM) to evaluate the new signal timing plans impact on the network performance. 


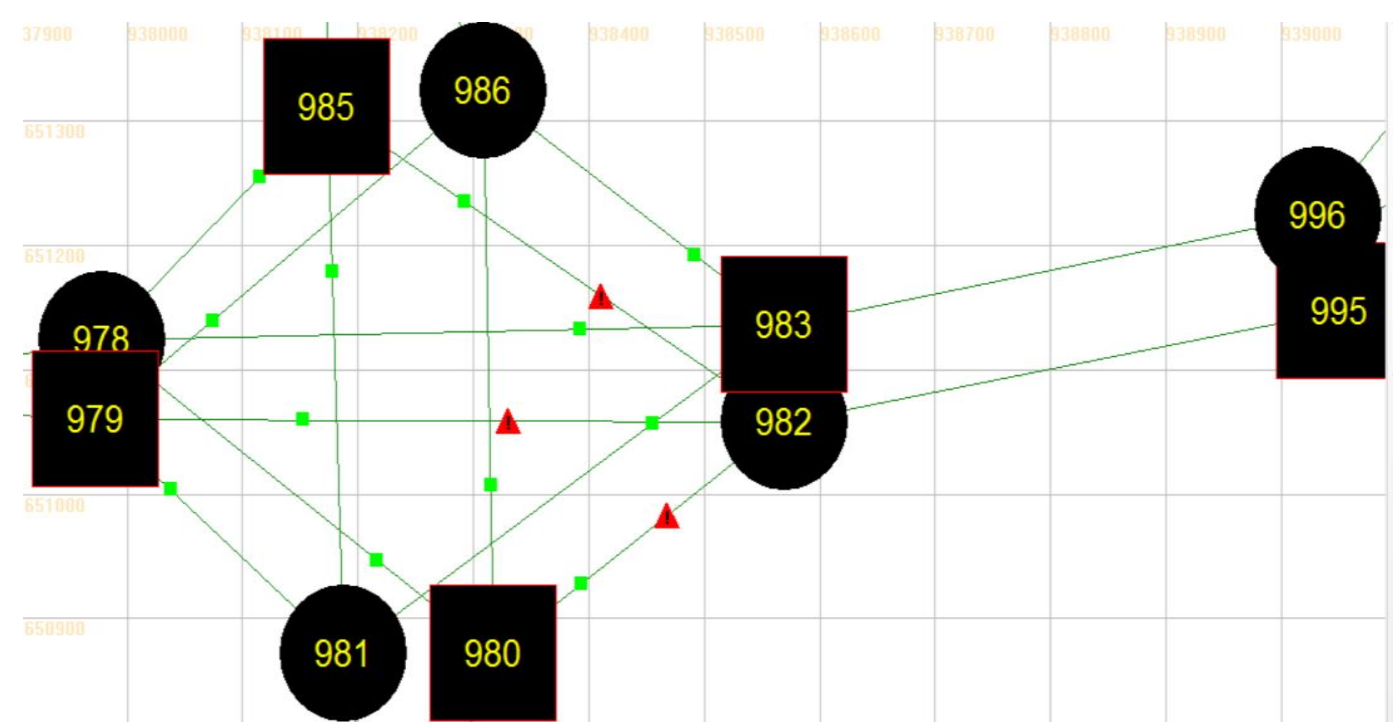

Figure 6-10 Drop the Incident Upstream Links' Capacities

\subsection{Microscopic Simulation of Special Signal Plans during Incidents}

The demands estimated for the three incident scenarios, as described above, were imported to VISSIM to model the impacts of special signal plans during incidents. Each incident scenario was evaluated first for the base condition with the signal control optimized for the normal conditions with no incidents. In the second scenario, the incident downstream and upstream signal green time were modified considering the models that predict the interactions between incidents and adjacent intersection control, derived as described in Section 6.2.

\subsubsection{Special Signal Timing Plan}

The implemented special signal timing plan was to divide the upstream signal incident movement green time (i.e., 61 seconds for a 45 minute incident) into two parts to reduce the spillback effect that block the green time at the end of a long green. The first green phase of the upstream main street movement was set to the estimated unconstrained 
green estimated using the models presented in Section 6.2. After the first green time, the upstream signal main street movement green was terminated and the greens of the cross street movements were turned on. Once the cross streets' movement green were terminated, a green time was assigned to the EB and WB green movements again as shown in the Figure. 6-11 (EB1 \& WB1). This new signal plan was set to start when the incident queue reaches the upstream signal and remained in effect until the incident queue was determined to have no impact on the upstream signal.

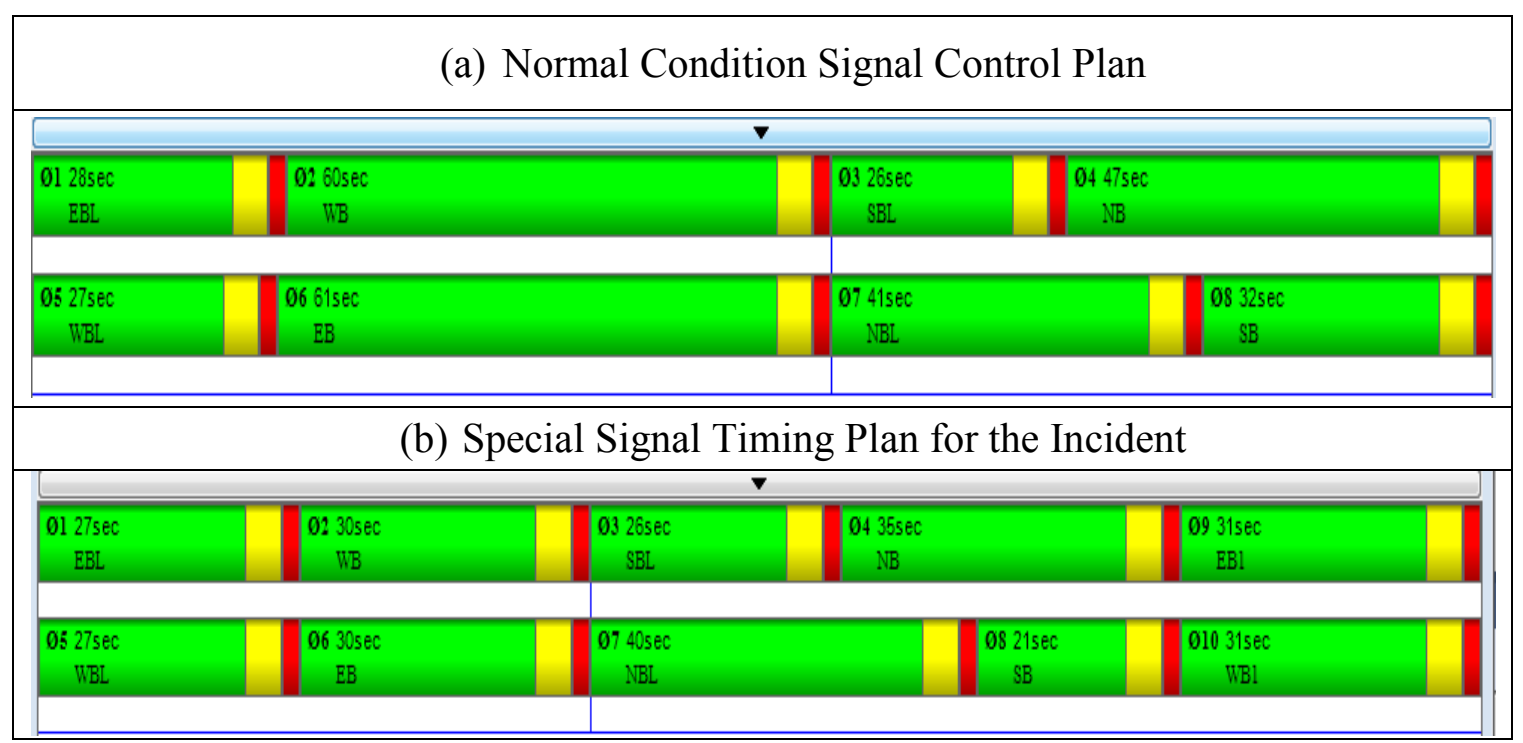

Figure 6-11 Signal Control Plan for Normal Day vs. Special Signal Control Plan

VISSIM microscopic simulation was used to estimate the impact of the special signal plan on the travel time delays during the incident for the whole network and Broward Blvd. EB direction (the incident direction) with normal signal control and incident special signal control. As shown in Table 6-5, the total network delay decreased from 456.31 to 412.69 vehicle-hours (11.3\% reduction), when the special signal control was implemented 
for 45 minutes. The results show that the implementation of the special signal plan can save 30 vehicle-hours of delay along Broward EB Boulevard. The 30 minutes incident duration results show that whole network delay decreases from 324 to 300 vehicle-hours ( $8 \%$ reduction) when the new signal timing was used during the incident. The results show the implementation of the special signal plan during the incident can save 16 vehicle-hours delay for the Broward EB Boulevard. The 15 minute incident duration results show that whole network delay decreases around 3 vehicle-hours when the new signal timing was used during the incident and that the incident can save 3 vehicle-hours delay through Broward EB Boulevard.

Table 6-5 Traffic Response Special signal Timing vs. Normal Signal Timing

\begin{tabular}{|c|c|c|c|c|c|c|}
\hline & \multicolumn{2}{|c|}{$\begin{array}{c}\text { 45 Minutes Incident } \\
\text { Delay (veh-hrs) }\end{array}$} & \multicolumn{2}{c|}{$\begin{array}{c}\text { 30 Minutes Incident } \\
\text { Delay (veh-hrs) }\end{array}$} & \multicolumn{2}{c|}{$\begin{array}{c}\text { 15 Minutes Incident } \\
\text { Delay(veh-hrs) }\end{array}$} \\
\hline Street & $\begin{array}{c}\text { Normal } \\
\text { Signal } \\
\text { Timing }\end{array}$ & $\begin{array}{c}\text { Special } \\
\text { Signal } \\
\text { Timing }\end{array}$ & $\begin{array}{c}\text { Normal } \\
\text { Signal } \\
\text { Timing }\end{array}$ & $\begin{array}{c}\text { Special } \\
\text { Signal } \\
\text { Timing }\end{array}$ & $\begin{array}{c}\text { Normal } \\
\text { Signal } \\
\text { Timing }\end{array}$ & $\begin{array}{c}\text { Special } \\
\text { Signal } \\
\text { Timing }\end{array}$ \\
\hline $\begin{array}{c}\text { Broward } \\
\text { Blvd }\end{array}$ & 121.17 & 91.49 & 87.77 & 71.50 & 53.40 & 49.89 \\
\hline $\begin{array}{c}\text { Network } \\
\text { Blvd }\end{array}$ & 456.31 & 412.69 & 324.19 & 300.14 & 197.46 & 194.10 \\
\hline
\end{tabular}

\subsection{Summary}

This chapter discussed the method used to assess incident responsive dynamic signal control. An important aspect of the assessment is to determine the capacity impacts of the interaction between the drop in capacity below demands at the midblock urban street segment location and upstream and downstream signalized intersection operations. A model was developed to estimate the drop in capacity at the incident location as a function 
of the number of blocked lanes, distance from downstream intersection, and green time to cycle length $(\mathrm{g} / \mathrm{C})$ ratio of the downstream signal. A second model was developed to estimate the reduction in the upstream intersection capacity due to the drop in capacity at midblock incident location as estimated by the first model. The second model estimates the drop in capacity of the upstream links feeding the incident locations as a function of incident duration time, volume to capacity (v/Ic) ratio at the incident location, and distance from an upstream signalized intersection.

Models were developed in this study based on data generated utilizing a microscopic simulation model that was calibrated for incident and no-incident conditions. In this study, the developed models were used to set the capacity during the incident conditions in the mesoscopic-based DTA tool to estimate the diversion during incidents. To supplement DTA-based analysis, regression models were developed to estimate the diversion rate due to urban street incidents. These regression models were combined with the DTA model to estimate the volume at the incident location and alternative routes. The developed models can estimate incident impacts on urban street facilities that can support the development and use of special signal plans based on incident attributes and urban network characteristics. Special signal timing plan was designed based on the unconstrained green time model that was diverted from the upstream signal maximum throughput models. The results indicate that the delay resulting from the implementation the traffic responsive signal control strategies can be reduced significantly. 


\section{CHAPTER 7}

\section{ESTIMATING INCIDENT IMPACTS ON URBAN STREET TRAVEL TIME}

\subsection{Introduction}

Assessing the impacts of incident management strategies on performance measures allows traffic management agencies to prioritize their investment in these strategies by supporting better planning for operations and operator decisions. There has been an increasing interest in analyzing the impacts of incident management performance on urban streets. The reduction of the impacts of incidents by applying incident management and signal control strategies is one of the main interests of transportation agencies.

The impacts of incident and incident management on mobility measures have been widely investigated for freeway facilities. Four methods have been used by transportation agencies for the following: empirical analysis, queuing analysis, shock wave analysis, and simulation modeling. Queuing and shockwave analysis methods have been successfully applied to the estimation of incident and bottleneck delays on freeway facilities (Zhang et al., 2010; Hong et al., 2013) (Hadi et al., 2007). As discussed in the literature review, these methods may not be accurate in terms of estimating delays for incidents on signalized urban streets without the consideration of the interactions between traffic signal operations and the capacity drop at the incident location.

Traffic simulation has also been used to estimate incidents and incident management impacts (Gomes et al., 2004; Crowe 2009). Such analysis is able to model the interactions between the drops in capacity due to incidents and intersection operations. 
However, the use of simulation models can be costly in terms of data collection, model input preparation, and calibration, especially when the incident management strategies need to be evaluated at the regional levels when the stochastic nature of incident features and locations need to be considered in the analysis, and when the incident impact has to be analysed for a long period of time (e.g., one year), and for real-time operations.

This chapter discusses the development and application of a method to estimate such impacts of support of planning for operations and operational processes. This chapter first discusses the extension of existing analytical procedures to allow better assessment of the impacts of incidents considering the interactions between the reductions in capacity below demands at midblock urban street segment locations and upstream and downstream signalized intersection operations, as explained in the previous chapter. This chapter also discusses the incorporation of the method in a data analytical tool and its use to inform the identification of a special signal timing plan to reduce incident impacts as part of a decision support system.

\subsection{Estimating Incident Impact on Delay}

This study develops a method to estimate incident delay using a combination of the delay due to queuing on the mid-block incident link and the increase in upstream intersections delay due to the reduction of the saturation flow rate or maximum throughput resulting from the queue spillback at the upstream intersection. The first component was calculated using deterministic queueing analysis equations, as is used in estimating incident delays on freeways. This method estimates the total delays based on incident duration, 
mean arrival rate (demand), and mean capacity under incident condition. The total delay due to lane blockage is calculated, as follows: (May, 2016).

$$
\begin{aligned}
T D & =\frac{\left(t_{R}\right)\left(t_{Q}\right)\left(\lambda-\mu_{R}\right)}{2} \\
t_{Q} & =\frac{\left(t_{R}\right)\left(\mu-\mu_{R}\right)}{(\mu-\lambda)}
\end{aligned}
$$

where

$$
\begin{aligned}
T D & =\text { total delay } \\
t_{Q} & =\text { average time in queue } \\
\mu & =\text { mean capacity under normal conditions, } \\
\mu_{R} & =\text { mean capacity under incident conditions, } \\
\lambda & =\text { mean arrival rate, and } \\
t_{R} & =\text { average incident duration. }
\end{aligned}
$$

The increase in the upstream intersection delay is calculated using the signalized intersection control delay method presented in the 2010 version of the Highway Capacity Manual (HCM 2010) (TRB Manual, 2010). The method calculates control delay as the sum of three components: uniform, incremental, and initial queue delays. An important parameter for calculating delay using this method is the capacity of the assessed lane group, which is normally calculated as the multiplication of the saturation flow rate and the effective green time divided by cycle length. To account for the spillback from the incident location, this study estimates upstream intersection capacity utilizing regression models as 
a function of incident attributes, volume-to-capacity ratio at the incident location, and upstream signal timing, as described in the next section.

\subsection{Capacity of Urban Street Segments}

As mentioned earlier, the saturation flow adjustment factor is a function of the number of lanes, number of lanes blocked by the incident, and coefficients related to incident severity. However, the adjustment factor does not address incident locations other than at the stop line. These incidents are expected to impact the signal operations. An incident occurring at a mid-block location can decrease the throughput of the incident link. If the queue from the incident spills back to the upstream link, it will decrease the upstream intersection throughput and consequently, the operations.

Chapter 6 discusses the reduction in the upstream intersection throughputs in the analysis period, which is a function of how far the incident is from the upstream intersection, the volume to capacity (v/Ic) ratio at the incident location, the incident duration, and the upstream intersection signal timing. The capacity at the incident location (Ic) is also affected by downstream intersection parameters, including how far the incident is from the downstream intersection, and also discusses the green to cycle-length $(\mathrm{g} / \mathrm{C})$ ratio and number of total and blocked lanes. Therefore, the developed regression models estimate upstream and downstream link capacities during incidents based on incident characteristics, upstream signal operations, and downstream signal operations. The first model estimates the lane-blockage capacity at the incident location with consideration of the impacts of the downstream signal queue spill back at different incident locations from 
the downstream signal. The second model estimates the reduction in the upstream intersection throughput due to incidents at different downstream locations. Both models can be used together to estimate capacities during incident conditions that can be used as inputs to the analytical delay equations described in the previous section. The developed models to estimate downstream (incident) link capacity and upstream intersection throughput are summarized and shown in Table 7-1.

Table 7-1 Regression Models to Estimate Interaction Between the Drop in Capacity due to Incidents and Upstream and Downstream Signal Operations

\begin{tabular}{|c|c|c|c|}
\hline $\begin{array}{c}\text { Blockage } \\
\text { Type }\end{array}$ & $\begin{array}{c}\text { Downstream } \\
\text { Signal g/C }\end{array}$ & Regression Model & $\mathbf{R}^{\mathbf{2}}$ \\
\hline $\begin{array}{c}\text { One-Lane } \\
\text { Blockage }\end{array}$ & 0.55 & $\mathrm{Ic}=0.7924 \mathrm{x}+1796.2$ & 0.911 \\
\cline { 2 - 4 } & 0.67 & $\mathrm{Ic}=0.6322 \mathrm{x}+2153.5$ & 0.941 \\
\hline $\begin{array}{c}\text { Two- } \\
\text { Lane } \\
\text { Blockage }\end{array}$ & 0.55 & $\mathrm{Ic}=6 \mathrm{E}^{-07} \mathrm{x}^{3}-0.0016 \mathrm{x}^{2}+1.2092 \mathrm{x}+633.45$ & 0.898 \\
\cline { 2 - 4 } & 0.67 & $\mathrm{Ic}=4 \mathrm{E}^{-07} \mathrm{x}^{3}-0.0011 \mathrm{x}^{2}+0.839 \mathrm{x}+706.85$ & 0.921 \\
\hline $\begin{array}{c}\text { Incident } \\
\text { Duration }\end{array}$ & $\begin{array}{c}\text { //Ic at Incident } \\
\text { Location }\end{array}$ & Intersection Maximum Throughput & $\mathbf{R}^{2}$ \\
\hline \multirow{2}{*}{$\begin{array}{c}15 \\
\text { Minutes }\end{array}$} & 1.13 & $\mathrm{SF}=0.4858 \mathrm{x}_{1}+1143.6$ & 0.845 \\
\cline { 2 - 4 } & 1.3 & $\mathrm{SF}=0.3317 \mathrm{x}_{1}+1146.7$ & 0.838 \\
\hline \multirow{2}{*}{$\begin{array}{c}35 \\
\text { Minutes }\end{array}$} & 1.5 & $\mathrm{SF}=0.3334 \mathrm{x}_{1}+994.31$ & 0.884 \\
\cline { 2 - 4 } & 1.13 & $\mathrm{SF}=0.4628 \mathrm{x}_{1}+1113.7$ & 0.831 \\
\hline \multirow{2}{*}{$\begin{array}{c}45 \\
\text { Minutes }\end{array}$} & 1.3 & $\mathrm{SF}=0.3242 \mathrm{x}_{1}+1048$ & 0.729 \\
\cline { 2 - 4 } & 1.13 & $\mathrm{SF}=0.2761 \mathrm{x}_{1}+928.35$ & 0.893 \\
\cline { 2 - 4 } & 1.3 & $\mathrm{SF}=0.4632 \mathrm{x}_{1}+1053.4$ & 0.827 \\
\hline
\end{tabular}




\subsection{Arterial Delay Estimation Accuracy Assessment Results}

In the developed model, incident delays were estimated as the sum of the incident link delays based on the queuing equations and the increase in upstream control delay due to spillback with consideration of the drop in saturation flow rates due to the incident according to the developed regression models in Table 7-1. The Highway Capacity Software (HCS) was used for the calculation of the control delay with the adjusted saturation flow rates as shown in Table 7-1. The resulting arterial incident delay was evaluated by comparing the results with incident delays estimated using VISSIM. The VISSIM model accounts for the effects of the interactions between upstream and downstream signal operations and midblock incident capacity drops since it models the queue spillbacks between different segments in the network. The scenario used in this comparison involves a midblock incident that results in a demand to capacity ratio of 1.13 at the incident location. Incidents at different locations were modeled in VISSIM (calibration of the VISSIM network explained in the previous chapter), and the additional delays due to the incidents were extracted based on the average of ten runs for the noincident conditions and ten runs for the incident conditions.

The network coded in the HCS was matched with the VISSIM network to provide a valid comparison. The calibrated VISSIM model with no incidents produced a saturation flow rate of $1854 \mathrm{veh} / \mathrm{hr} /$ lane, which was used as the input saturation flow rate in the HCS analysis. The base analysis period in the HCM, and thus HCS analysis, is fixed at 15 minutes. The simulated incident duration in this study is 35 minutes. Therefore, during 
the incident condition, the saturation flow rates within the first two periods (30 minutes) were calculated based on the regression equations presented in Table 7-1. For the third period (between 30 and 45 minutes), after the incident occurrence, in order to account for the additional 5 minutes of the incident duration, the saturation flow was calculated as a weighted average of the saturation rate during the incident in the first 5 minutes of the period and the no-incident saturation flow rate during the last 10 minutes of the period. This accounted for the full 35-minute period of the incident. The incident delays in the HCS and VISSIM were calculated as the difference between the delay with incident and without incident. A comparison was also made of the estimation of the incident delay using the queuing equation by itself to determine the delay at the midblock incident location, as has been used for freeway incidents. The incident delay comparison results are shown in Figure 7-1.

Figure 7-1 shows that the incident delay decreased by moving the incident from the upstream signal toward the downstream, which is expected due to the reduction in the impact on the upstream intersection throughputs due to queue spillbacks. The results show that the use of the combination of deterministic queuing and the HCM equations procedure with the modified saturation flow of Table 1 to calculate incident delays produced results that are closer to the delays estimated by the microsimulation models, compared to the results obtained based on the deterministic queuing procedure by itself. This is particularly true for incidents located at closer distances (up to 400-500 $\mathrm{ft}$ ) from the upstream intersection for the $\mathrm{v} / \mathrm{Ic}$ ratio of this scenario, which is 1.13 . Beyond this point, the VISSIM 
simulation shows higher impacts of incidents on the delay of the upstream intersection, compared to the HCM procedure, even with the modified saturation flow rates.

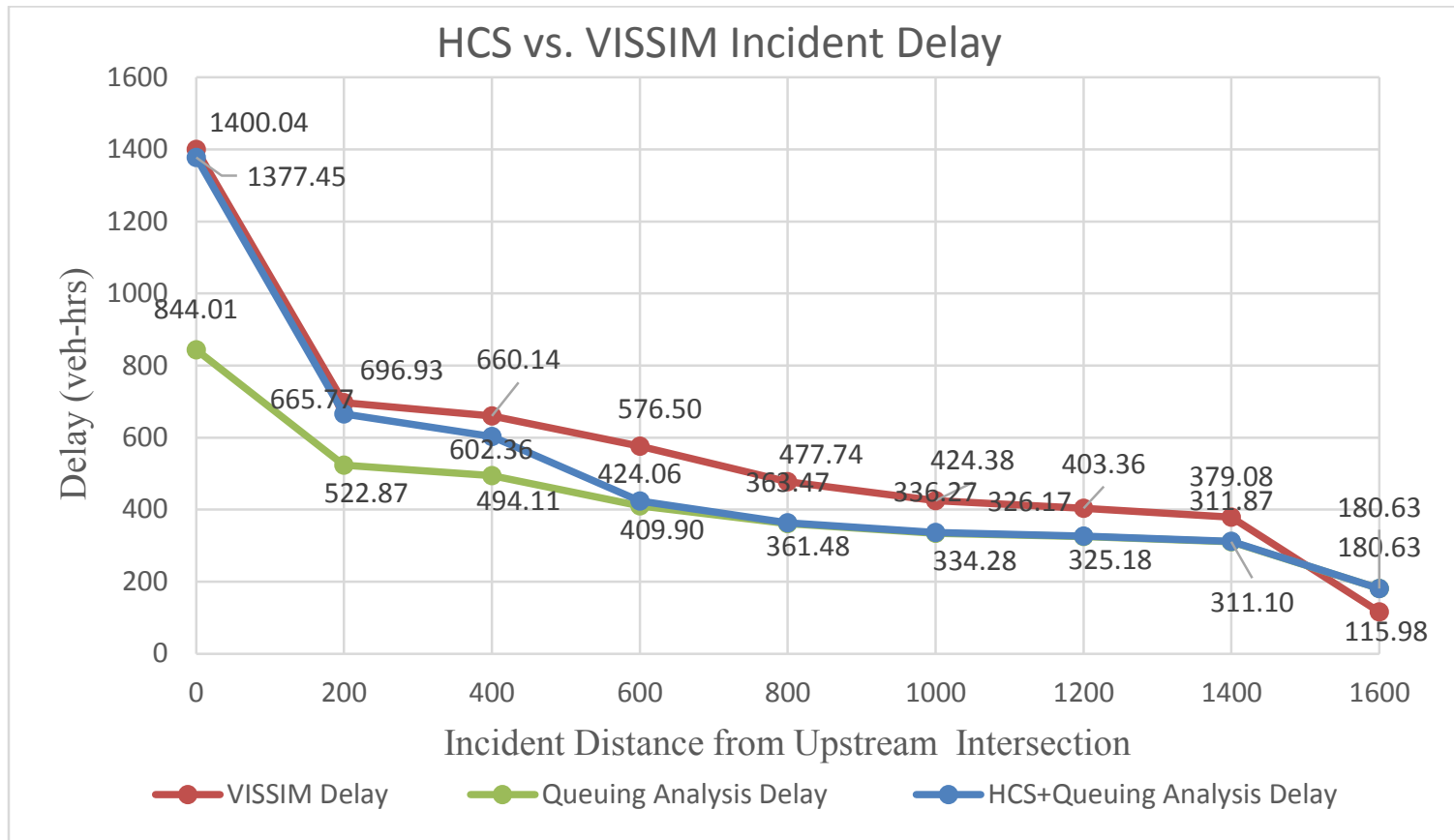

Figure 7-1 Comparison of Incident Delay Using the Proposed Method, Queuing Analysis, and VISSIM

This may be due to the stochastic nature of VISSIM, which better reflects the randomness of traffic arrivals and dissipations. The HCM procedures do not fully account for this randomness, and thus it may underestimate the impacts under certain conditions.

\subsection{Model Utilization for Decision Support}

As stated earlier, transportation agencies are interested in assessing the impacts of various strategies to reduce incident impacts on signalized urban arterials, including the application of special signal plans during incidents. Currently, this can only be achieved by utilizing simulation models that consider the interaction between the operations at the 
incident location and upstream and downstream signals. The model developed in this study can be used to support analytical assessments of such strategies by incorporating the impacts of such interactions.

Recognizing the need discussed above, the method developed in this study has been implemented in a web-based data analytics tool, referred to as the ITS Data Capture and Performance Management (ITSDCAP) tool, which was developed for the Florida Department of Transportation (FDOT) by a research team. ITSDCAP is a data analytics tool that has a number of modules for utilizing real-time and archived data to support agency operations. The implemented method in ITSDCAP allows the user to better estimate of the impacts of incidents on urban arterials and potential strategies such as incident management activities that reduce the lane blockage duration and the implementation of a signal timing plan in response to incident conditions.

To illustrate the utilization of the ITSDCAP implementation, the methodology was applied to a real-world case study of an incident that occurred at West Oakland Park Boulevard and the I-95 southbound interchange. The incident location was 0.1 mile from the upstream signal and 0.25 mile from the downstream signal. The event started at 8:18 a.m. and ended at 9:07 a.m., with a total incident duration of 49 minutes. The v/Ic ratio at the incident location was 1.44 , resulting in a queue spillback to the upstream signal and capacity constraints at the intersection during portions of the green times of the feeder links to the incident link. This case study demonstrates the use of the developed delay estimation methodology to decide on a special signal timing plan to reduce the spillback impacts. To 
reduce the spillback impacts, the special signal timing plan can be developed by: 1) increasing the green time at the downstream intersection to eliminate backup from the downstream intersection to the incident location, which can further reduce the available capacity at the incident location, and 2) the upstream intersection green and cycle length should be modified to limit the amount of traffic leaving the upstream in each cycle to a level that does not cause spillbacks that constrain the amount of traffic that can leave the upstream stop line in a cycle. However, it was determined from the case study incident that the downstream intersection effective green to capacity $(\mathrm{g} / \mathrm{C})$ ratio is 0.65 , and the incident occurred 0.25 mile from the downstream signal. Applying the downstream intersection models in Table 7-1 indicates that for such parameters, there is no interaction between the queue length from the downstream signal and the operations at the incident condition, and thus the incident capacity is not affected by the queues from the downstream intersection. For this reason, no changes are required for downstream signal control timing. However, when applying the models in Table 7-1, the results indicate a reduction in throughput of the upstream signal due to the incident. Thus, a special signal plan was designed to eliminate the constrained green time, in which traffic from feeder links are blocked by queues from the incident link.

The adjustment to the signal timing was made based on the upstream intersection throughput model developed in this study, and the impacts of the adjusted timing were evaluated using VISSIM simulation. Also, VISSIM results were compared with the proposed delay calculation method utilizing the HCM procedure implemented in the 
ITSDCAP. The adjustment to the green time made in this study required an estimation of the unconstrained green for upstream movements. The remaining green will be constrained by the queue spillback. Thus, providing green times equal to the unconstrained green will minimize the lost green due to the spillback. The unconstrained green is calculated based on the saturation flow rate provided in Table 6-4.

\subsection{Model Utilization for Decision Support}

As stated earlier, transportation agencies are interested in assessing the impacts of various strategies to reduce incident impacts on signalized urban arterials, including the application of special signal plans during incidents. Currently, this can only be achieved by utilizing simulation models that consider the interaction between the operations at the incident location and upstream and downstream signals. The model developed in this study can be used to support analytical assessments of such strategies by incorporating the impacts of such interactions.

The method developed in this study has been implemented in a web-based data analytics tool referred to as the ITS Data Capture and Performance Management (ITSDCAP) tool, developed for the Florida Department of Transportation (FDOT) by a research team. ITSDCAP is a data analytics tool that has a number of modules for utilizing real-time and archived data to support agency operations. The implemented method in ITSDCAP allows the user to better estimate the impacts of incidents on urban arterials and the potential strategies, such as incident management activities that reduce the lane 
blockage duration and the implementation of signal timing plans in response to incident conditions.

To illustrate the utilization of the ITSDCAP implementation, the methodology was applied to a real-world case study of an incident that occurred at West Oakland Park Boulevard and the I-95 southbound interchange. The incident location was 0.1 mile from the upstream signal and 0.25 mile from the downstream signal. The event started at 8:18 a.m. and ended at 9:07 a.m., with a total incident duration of 49 minutes. The V/Ic ratio at the incident location was 1.44 , resulting in queue spillback to the upstream signal and capacity constraints at the intersection during portions of the green times of the feeder links to the incident link. This case study demonstrates the use of the developed delay estimation methodology to decide on a special signal timing plan to reduce the spillback impacts. To reduce the spillback impacts, the special signal timing plan can be developed by: 1) increasing the green time at the downstream intersection to eliminate backup from the downstream intersection to the incident location, which can further reduce the available capacity at the incident location, and 2) the upstream intersection green and cycle length should be modified to limit the amount of traffic leaving the upstream in each cycle to a level that does not cause spillback that constrains the amount of traffic that can leave the upstream stop line in a cycle. However, it was determined from the case study incident that the downstream intersection effective green to capacity $(\mathrm{g} / \mathrm{C})$ ratio is 0.65 , and the incident occurred 0.25 mile from the downstream signal. Applying the downstream intersection models shown in Table 1 indicates that for such parameters, there is no 
interaction between the queue length from the downstream signal and the operations at the incident condition, and thus the incident capacity is not affected by the queues from the downstream intersection. For this reason, no changes are required for downstream signal control timing. However when applying the models in Table 1, the results indicate a reduction in the throughput of the upstream signal due to the incident. Thus, a special signal plan was designed to eliminate the constrained green time, in which traffic from feeder links are blocked by queues from the incident link.

The adjustment to the signal timing was made based on the upstream intersection throughput model developed in this study, and the impacts of the adjusted timing were evaluated using VISSIM simulation. Also, VISSIM results were compared with the proposed delay calculation method utilizing the HCM procedure implemented in the ITSDCAP. The adjustment to the green made in this study required an estimation of the unconstrained green time for upstream movements. The remaining green will be constrained by the queue spillback. Thus, providing green times equal to the unconstrained green will minimize the lost green due to the spillback. The unconstrained green time is calculated based on the saturation flow rate provided in Table 6-4.

Table 7-2 shows that both VISSIM and the HCM-based methods developed in this study and applied in ITSDCAP estimated a reduction in delay due to the updated signal timing during incidents. The HCS-based method estimated a reduction in delay resulting from the new signal timing plan to be around 3,414 vehicle-hours, whereas the VISSIM 
shows that the new signal timing saving in delay is 4,018 vehicle-hours. This confirms that the developed method and VISSIM estimation of the benefits are consistent.

Table 7-2 Network Delay Saving (Vehicle-hrs) due to Upstream Signal Timing Plan Adjustment During the Incident Condition in VISSIM and the Proposed Analytical Model

\begin{tabular}{|c|c|c|c|c|}
\hline \multicolumn{5}{|c|}{ VISSIM Models } \\
\hline Scenario & \multicolumn{2}{|c|}{$\begin{array}{c}\text { Network Total Delay } \\
\text { (veh-hrs) }\end{array}$} & \multicolumn{2}{|c|}{$\begin{array}{l}\text { Signal Adjustment Saving } \\
\text { Delay (veh-hrs) }\end{array}$} \\
\hline $\begin{array}{l}\text { Incident without } \\
\text { Signal Adjustment }\end{array}$ & \multicolumn{2}{|c|}{10703} & \multirow{2}{*}{\multicolumn{2}{|c|}{4018}} \\
\hline $\begin{array}{c}\text { Incident with } \\
\text { Signal Adjustment }\end{array}$ & \multicolumn{2}{|c|}{6684} & & \\
\hline \multicolumn{5}{|c|}{ Proposed Method Combination of Queuing and HCM Equations } \\
\hline Scenario & $\begin{array}{c}\text { Upstream } \\
\text { Signal Delay } \\
\text { (HCM } \\
\text { Equations) } \\
\text { (veh-hrs) }\end{array}$ & $\begin{array}{c}\text { Queuing } \\
\text { Analysis } \\
\text { Delay } \\
\text { (veh-hrs) }\end{array}$ & $\begin{array}{c}\text { Total } \\
\text { Delay } \\
\text { (veh-hrs) }\end{array}$ & $\begin{array}{c}\text { Signal } \\
\text { Adjustment } \\
\text { Saving Delay } \\
\text { (veh-hrs) }\end{array}$ \\
\hline $\begin{array}{c}\text { Incident without } \\
\text { Signal Adjustment }\end{array}$ & 7008 & 2718 & 9726 & \multirow{2}{*}{3414} \\
\hline $\begin{array}{c}\text { Incident with } \\
\text { Signal Adjustment }\end{array}$ & 5425 & 887 & 6312 & \\
\hline
\end{tabular}

\subsection{Summary}

The estimation of incident and incident management strategy impacts on urban street performance is important to a successful planning for operations and operations of active transportation management strategies. This chapter described the development and application of a new model to estimate the impacts of incidents and incident management strategies on urban street facilities. The developed capacity reduction models were used to estimate delay due to an urban street incident. The delay was calculated as a combination 
of the delay due to queuing on the incident link and the increase in upstream intersection control delays due the reduction in maximum throughputs resulting from queue spillback to the upstream intersection. A comparison with microscopic simulation modeling results showed that the delay estimated using the combination of the increase in control delay based on the reduction in capacity estimated by the developed models and deterministic queuing at the incident location produced better results than using the deterministic queuing procedure by itself for estimating delays.

The developed method was successfully incorporated in a data analytic tool to support agency operation decisions and is demonstrated to be able to support agency assessment of the effectiveness of incident management and associated signal control strategies. 


\section{CHAPTER 8}

\section{CONCLUSION AND RECOMMENDATIONS FOR FUTURE STUDIES}

ATM strategies are important to address the transportation system problems. There is a strong trend to invest in ATM strategies on urban streets (signalized arterials) since such strategies have already been implemented on large proportions of urban freeway segments. The potential benefits of ATM strategies include mobility improvement, unreliability reduction, environmental impact reduction, and safety improvement. Methods are needed to assess the impact of these ATM strategies.

The advancement of transportation system technologies and strategies, the increase in the availability of data, and the realization of the importance of planning for operations have increased the consideration of the need for integrating different modeling tools for more effective assessment of ATM strategies. An important aspect of this consideration is the recognition of the potential for use of simulation-based DTA to evaluate ATM strategies on urban streets. The reason is that simulation-based DTA can better capture the dynamics of traffic operations, compared to traditional methods such as static traffic assignment (STA) with simple analytical traffic flow equations. Although DTA has the potential to better replicate real-world conditions, other tools such as signal control optimization tools and microscopic simulation tools are needed in conjunction with the DTA tools to produce a comprehensive and effective analysis of advanced strategies. This study demonstrates the utilization of a multi-resolution approach that combines data 
analytics, DTA modeling, analytical modeling, and microscopic simulation to assess the impacts of ATM strategies on urban street traffic. The following subsections are the conclusions based on the results of this research and recommendations for future studies.

\subsection{Conclusions}

This study demonstrates the need for and utilization of a multi-resolution and multiscenario modeling approach to support the evaluation and design of ATM strategies. Most ATM strategies are effective in relieving congestion during non-typical days and patterns with high demands, incidents with different severity levels, and adverse weather conditions. This research highlights the importance of modeling different traffic patterns when assessing the benefits of dynamic signal control that is responsive to traffic and incident conditions. The days that correspond to different percentiles of traffic, such as the $50^{\text {th }}, 75^{\text {th }}$, and $95^{\text {th }}$ percentiles were determined and modeled using a mesoscopicsimulation-based DTA. The origin-destination demands for the three levels were determined utilizing an ODME process based on a seed matrix and traffic counts for the three days. It was determined that current methods of calibrating the demands utilizing ODME modules based on segment counts is not adequate enough to produce accurate estimates of the turning movement counts and that the inclusion of turning movement counts as an input to the ODME is necessary.

The study then demonstrated the use of the DTA model with this setup to produce turning movement volumes for different demand levels to input into signal optimization and microscopic simulation modeling tools to assess the impact of dynamic signal control. 
The Highway Capacity Software (HCS) was used to optimize the signal timing for different traffic patterns, and the resulting signal timings were evaluated using microscopic simulation to evaluate the traffic response signal control performance. The results indicate that the delay resulting from the implementation the traffic response signal control strategies can be reduced significantly and that this method can be used as part of planning for operations to evaluate and design strategies.

This study also investigated the method used to assess incident responsive dynamic signal control. An important aspect of the assessment is to determine the capacity impacts of the interaction between the drop in capacity below demands at the midblock urban street segment location and the upstream and downstream signalized intersection operations. A model was developed to estimate the drop in capacity at the incident location as a function of the number of blocked lanes, distance from downstream intersection, and $\mathrm{g} / \mathrm{C}$ ratio of the downstream signal. A second model was developed to estimate the reduction in the upstream intersection capacity due to the drop in capacity at the midblock incident location as estimated by the first model. The second model estimates the drop in capacity of the upstream links feeding the incident locations as a function of incident duration time, v/c ratio at the incident location, and distance from an upstream signalized intersection. The developed regression models show significant relationships between the drops in incident capacity and the drops in upstream saturation flow as dependent and independent variables in the model. The focus of this study was on a three-lane arterial facility because threelane facilities are the most common type of principal urban street facilities. However, the 
second model that estimates upstream intersection flow rates is applicable to facilities with a different number of lanes. The developed capacity reduction models were used to estimate delay due to an urban street incident. The delay was calculated as a combination of the delay due to queuing on the incident link and the increase in upstream intersection control delays due the reduction in maximum throughputs resulting from queue spillback to the upstream intersection. A comparison with microscopic simulation modeling results showed that the delay estimated using the combination of the increase in control delay based on the reduction in capacity estimated by the developed models and deterministic queuing at the incident location produced better results than using the deterministic queuing procedure by itself for estimating delays.

The developed method to estimate urban street incident impacts was then implemented as part of the multi-resolution modeling to refine the DTA modeling and as an input to the highway capacity manual-based modeling of incident and incident responsive management impacts. To supplement the DTA modeling for this purpose, additional regression models were developed to estimate diversion due to urban street incidents. These regression models were combined with the DTA model to estimate the volumes at the incident location and alternative routes. The produced volumes during the incident were then used as inputs to microscopic simulation for more detailed analysis and to demonstrate the benefits of special signal plans during incident conditions. 


\subsection{Recommendations for Future Works}

As mentioned earlier, this study aims to explore the effectiveness of different levels of modeling when used separately and in combination with the modeling of arterial ATM strategies. A number of research and development topics are recommended to extend the results of this study, as listed below:

- This study developed a method to evaluate signal plans that are responsive to traffic and incidents. The methods developed in this study can also be used to select the signal timing plans for different traffic demand levels. There is a need for methods to optimize signal timing plans under congested conditions utilizing the incident impact model developed in this study.

- Multi-resolution, multi-scenario modeling requires extensive effort from the user and can benefit from developing automated tools to reduce the effort. There is a need to develop tools that automate the conversion of real-world data into a format that can be used to support the modeling process and translate information between different modeling tools.

- The utilization of the ODME process shows that the estimated O-D matrix is still not able to produce reasonably good link volumes at certain locations compared to real-world data, with the utilized initial (seed) demand matrix. Therefore, a manual adjustment of the initial O-D matrix was made before running the ODME process. Further research on the ODME process is needed, possibly combined with a method to disaggregate the zones and the associated zone connecters to improve the 
simulation-based DTA process. In the future, it is recommended that a method is developed to automatically disaggregate the zones into subzones to produce realistic connections to the network.

The method developed in this study to estimate diversion includes the use a combination of DTA and regression models developed based on real-world data. Previous efforts have used non-iterative assignments that reassign vehicles that have access to alternative routes, if sufficient improvement in travel time can be realized due to this diversion. In this study, it was not possible to compare the performance of the developed method versus the method utilized in the literature due to a bug in the utilized DTA tool that did not allow the varying the level of improvement in travel time that motivates drivers to divert to an alternative route. It is recommended that such a comparison is made in future studies. 


\section{REFERENCES}

Abdel-Rahim, A., A. Krings and M. Dixon (2011). Integrating Clarus Data in Traffic Signal System Operation: A Survivable Real-time Weather-responsive System.

Abdel-Rahim, A. S., W. C. Taylor and A. Bangia (1998). Analysis of Corridor Delay Under Scats Control. ITS America 8th Annual Meeting and Exposition: Transportation Technology for Tomorrow: Conference Proceedings.

Abdi, A., A. Mehara, S. Monajjem, E. Sadeghvaziri (2014). Simulation Modeling of Dynamic Response of Vehicles to Different Types of Speed Control Humps. American Society of Civil Engineers (ASCE) T\&DI Congress 2014: pp. 533-542

AG, P. P. T. V. (2007). VISSIM version 5.0 User Manual.Karlsruhe Germany.

Agbolosu-Amison, S., A. Sadek and W. ElDessouki (2004). "Inclement Weather And Traffic Flow At Signalized Intersections: Case Study from Northern New England." Transportation Research Record: Journal of the Transportation Research Board(1867): 163-171.

Agbolosu-Amison, S., A. Sadek and B. Henry (2005). Factors Affecting Benefits of Implementing Special Signal Timing Plans for Inclement Weather Conditions. Transportation Research Record: Journal of the Transportation Research Board(1925): 146-155.

Aghdashi, B., S. Khazraeian, J. Trask, M. Hadi, N. Rouphail, "Incorporating Adaptive (Traffic Responsive) Ramp Metering in the Highway Capacity Manual Context", Proceedings of the 96th Annual Meeting of the Transportation Research Board, Washington, D.C., January 2017.

Ahmad, I., Suksawang, N., Sobhan, K., Corven, J., Sayyafi, E. A., Pant, S., \& Martinez, F. (2015). Develop Epoxy Grout Pourback Guidance and Test Methods to Eliminate Thermal/Shrinkage Cracking at Post-Tensioning Anchorages: Phase II. Transport Research International Documentation (TRID), Florida Department of Transportation.

Al-Kaisy, A. and Z. Freedman (2006). Weather-Responsive Signal Timing: Practical Guidelines. Transportation Research Record: Journal of the Transportation Research Board(1978): 49-60. 
Al-Nuaimi, H., R. Ayers and K. Somasundaraswaran (2013). Traffic Flow Modeling For An Urban Arterial Road in Brisbane City. Proceedings of the 14th Road Engineering Association of Asia and Australasia Conference (REAAA 2013), Road Engineering Association of Malaysia (REAM).

Allegheny (2011). Robinson Town Centre Boulevard/Summit Drive Park SINC Project Summary.

Alluri, P., A. Gan, D. Saha, L. Fernandez, E. Sadeghvaziri (2017). Evaluation of Signage Alternatives for Express Lane Facilities. The National Center for Transportation Systems Productivity and Management (NCTSPM), Contract \# DTRT12GUTC12 with USDOT Office of the Assistant Secretary for Research and Technology (OST-R), Final Report, January 2017.

Alvarez, P. and M. Hadi (2010). Use of ITS Data to Calibrate Microscopic Simulation Models for Incident Conditions. Submitted to the Summer Meeting of the Traffic Flow Theory and Characteristics Committee (AHB45) of the Transportation Research Board and Consideration for Publication at Transportation Research Part C.

Avetisyan, H. G., E. Miller-Hooks, S. Melanta and B. Qi (2014). Effects of Vehicle Technologies, Traffic Volume Changes, Incidents and Work Zones on Greenhouse Gas Emissions Production. Transportation research part D: transport and environment 26: 1019.

Balke, K. and D. Gopalakrishna (2013). Utah DOT Weather Responsive Traffic Signal Timing.

Banerjee and T. Frances (2001). Preliminary Evaluation Study of Adaptive Traffic Control System. ITS Benefits Database Entry.

Baqersad, M., Mohammadafzali, M., Choubane, B., Holzschuher, C., Hamedi, A. and Ali, H. (2017). "Precision Assessment of the Florida Texture Meter in Hot Mix Asphalt." ASCE's Journal of Transportation Engineering, Part B: Pavements, in press.

Baqersad, M., Hamedi, A., Mohammadafzali, M. and Ali, H., 2017. Asphalt Mixture Segregation Detection: Digital Image Processing Approach. Advances in Materials Science and Engineering, 2017 
Baqersad, M., Sayyafi, E. A., \& Bak, H. M. (2017). State of the Art: Mechanical Properties of Ultra-High Performance Concrete. Civil Engineering Journal, 3(3), 190-198.

Bhide, V. (2005). Strategies for Incident Management in an Urban Street Network.

Birst, S. and A. Smadi (2000). An Evaluation of ITS for Incident Management in SecondTier Cities: A Fargo, ND Case Study. ITS America 10th Annual Meeting and Exposition: Revolutionary Thinking, Real Results.

Bond, A., M. Grant, J. Bauer, S. Miller, B. Bowen, L. Martin and A. Giragosian (2013). Programming for Operations: MPO Examples of Prioritizing and Funding Transportation System Management \& Operations Strategies.

Bonneson, J. A. (2014). Urban Street Reliability Engine User Guide, Strategic Highway Research Program 2 Project L08.

Botha, J. L. and T. R. Kruse (1992). Flow Rates At Signalized Intersections Under Cold Winter Conditions. Journal of transportation engineering 118(3): 439-450.

Burghout, W. (2004). Hybrid microscopic-mesoscopic traffic simulation.

Caliper. (2015). TransModeler Traffic Simulation Software. from http://www.caliper.com/transmodeler/simulation.htm.

Carrion, C. and D. Levinson (2012). Value of travel time reliability: A review of current evidence.Transportation research part A: policy and practice 46(4): 720-741.

Carson, J. L. (2009). Traffic Incident Management Resource Management.

Chang, G. L. and S. Rochon (2006). Performance Evaluation of CHART-the Real Time Incident Management System in Year 2006. Maryland Department of Transportation.

Chiu, Y.-C. and B. Bustillos (2009). A Gap Function Vehicle-Based Solution Procedure For Consistent and Robust Simulation-Based Dynamic Traffic Assignment. Transportation Research Board 88th Annual Meeting. 
Chiu, Y.-C., L. Zhou and H. Song (2010). Development and Calibration of the Anisotropic Mesoscopic Simulation Model For Uninterrupted Flow Facilities. Transportation Research Part B: Methodological 44(1): 152-174.

Citilabs (2011). Cube Voyager Reference Guide Version 5.1.

Crowe, J. M. (2009). The Calibration, Validation, and Comparison of VISSIM Simulations Using The Two-Fluid Model, University of Central Florida Orlando, Florida.

Day, C. M., D. M. Bullock, H. Li, S. M. Remias, A. M. Hainen, R. S. Freije, A. L. Stevens, J. R. Sturdevant and T. M. Brennan (2014). Performance Measures for Traffic Signal Systems: An Outcome-Oriented Approach.

Dia, H. and N. Cottman (2006). Evaluation of Arterial Incident Management Impacts Using Traffic Simulation. Intelligent Transport Systems, IEE Proceedings, IET.

Dong, J. and H. Mahmassani (2009). Flow Breakdown and Travel Time Reliability.Transportation Research Record: Journal of the Transportation Research Board (2124): 203-212.

Dutta, U., J. Lynch, B. Dara and S. Bodke (2010). Safety Evaluation of the SCATS Control System.

EconomicDevelopmentResearchGroup, I., I. Cambridge Systematics, I. International, T. T. A. M. T. Institute and I. Weris (2013). Development of Tools for Assessing Wider Economic Benefits of Transportation.

FDOT (2006). Annual Report Smart Sunguide TMC.

M. Fesharaki, (2016). An Investigation on the Effect of Rail Corrugation on Track Response. International Journal of Science and Engineering Applications, Volume 5 Issue 7.

FHWA. (2016). Adaptive Signal Control Technology. from https://www.fhwa.dot.gov/innovation/everydaycounts/edc-1/asct.cfm. 
Ghaman, R., D. Gettman, L. Head and P. B. Mirchandani (2002). Adaptive control software for distributed systems. IECON 02 [Industrial Electronics Society, IEEE 2002 28th Annual Conference of the], IEEE.

Gillam, W. and R. Wilhill (1992). UTC and Inclement Weather Conditions. Road Traffic Monitoring, 1992 (IEE Conf. Pub. 355), IET.

Glassco, R. A., A. Proper, V. Shah and K. Wunderlich (1997). Studies of Potential Intelligent Transportation Systems Benefits Using Traffic Simulation Modeling-Volume 2.

Gomes, G., A. May and R. Horowitz (2004). Congested Freeway Microsimulation Model Using VISSIM. Transportation Research Record: Journal of the Transportation Research Board(1876): 71-81.

Goodwin, L. C. (2002). Weather Impacts On Arterial Traffic Flow. The Road Weather Management Program FHWA, Washington.

Goodwin, L. C. and P. Pisano (2003). Best practices for road weather management. Road Weather.

Gordon, R. L., R. A. Reiss, H. Haenel, E. R. Case, R. L. French, A. Mohaddes and R. Wolcott (1996). Traffic Control Systems Handbook-Revised Edition 1996.

Hadi, M., L. Shen, C. Zhan, Y. Xiao, S. Corbin and D. Chen (2007). Use of Operation Data to Evaluate the Benefits and Costs of Advanced Traffic Management Components By.

Hadi, M., P. Sinha and A. Wang (2007). Modeling Reductions in Freeway Capacity Due To Incidents In Microscopic Simulation Models. Transportation Research Record: Journal of the Transportation Research Board(1999): 62-68.

Hadi, M., P. Sinha and A. Wang (2007). Modeling Reductions in Freeway Capacity Due to Incidents in Microscopic Simulation Models. Transportation Research Record: Journal of the Transportation Research Board.

Hadi, M. and Y. Xiao (2015). Framework for Multi-Resolution Analyses of Advanced Traffic Management Strategies. 
Hadi, M., Xiao, Y., Tao, W., Fakharian Qom, S., Massahi, A., Azizi, L., Jia J., and. Iqbal M. S. (2016). Framework for Multi-Resolution Analyses of Advanced Traffic Management Strategies.

Hadi,M., Y. Xiao, H. Ozen and P. Alvarez (2008). Evaluation Tools to Support ITS Planning Process: Development of a Sketch Planning Tool in FSUTMS/Cube Environment.

Halkias, J. and M. Schauer (2004). Red light, Green light. Public roads 68(3).

Harris, J. T. (2005). Benefits of retiming traffic signals. ITE 2005 Annual Meeting and Exhibit Compendium of Technical Papers.

HCM (2010). HCM2010. Transportation Research Board, National Research Council, Washington, DC.

Hellernan, B. (2010). Managed Motorways in the Netherlands. Presentation to the FGD Scan Team, Centre for Transport and Navigation.

Hong, C., Y. Yue-sheng and Z. Bin (2013). A Capacity Assessment Method on Urban Expressway after Traffic Incident. Procedia-Social and Behavioral Sciences 96: 19211928.

Hossan, M., Asgari, H. and X. Jin (2016). "Investigating Preference Heterogeneity in Value of Time (VOT) and Value of Reliability (VOR) Estimation for Managed Lanes". Transportation Research Part A: Policy and Practice (94): pp. 638-649. https://doi.org/10.1016/j.tra.2016.10.022

Jin, X., Hossan, M., Asgari, H. and K. Shams (2017). "Incorporating Attitudinal Aspects in Roadway Pricing Analysis". Transport Policy, corrected proof, in press, https://doi.org/10.1016/j.tranpol.2017.03.020

Hutton, J. M., C. D. Bokenkroger and M. M. Meyer (2010). Evaluation of an adaptive Traffic Signal System: Route 291 in Lee’s summit, Missouri.

INRO. (2016). Dynameq, Traffic Simulation for Wide-Area Networks. from https://www.inrosoftware.com/en/products/dynameq/. 
International, I. (2012). Benefits of Florida's traffic signal retiming. from http://www.itsinternational.com/categories/utc/features/benefits-of-floridas-traffic-signalretiming/.

Izzy, K. (2012). NYC's Adaptive Congestion Management System. New York Minutes.

Jensen, M., C. Cluett, K. Wunderlich, A. Deblasio and R. Sanchez (2000). Metropolitan Model Deployment Initiative: Seattle Evaluation Report.

Jones, S. L., A. J. Sullivan, N. Cheekoti, M. D. Anderson and D. Malave (2004). Traffic Simulation Software Comparison Study, University Transportation Center for Alabama.

Kang, S. and D. W. Gillen (1999). Assessing the Benefits And Costs Of Intelligent Transportation Systems: Ramp Meters, California PATH Program, Institute of Transportation Studies, University of California at Berkeley.

Khattak, A. J., X. Wang, H. Zhang and M. Cetin (2007). Primary and Secondary Incident Management: Predicting Durations In Real Time. Contract 2009.

Khazraeian, S. Y. Xiao, M. Hadi, "Application of the Upcoming HCM Managed Lane Procedure to Pylon-Separated Managed Lane Analyses", Proceedings of the 94th Annual Meeting of the Transportation Research Board, Washington, D.C., January 2015.

Khazraeian, S., M. Hadi, Y. Xiao, "Detection of Freeway Incidents Based on Vehicle Acceleration Measurements Using Connected Vehicle Data," Proceedings of the 95th Annual Meeting of the Transportation Research Board, Washington, D.C., January 2016.

Khazraeian, S., M. Hadi, Y. Xiao, "Assessment of the Benefits of Queue Warning in a Connected Vehicle Environment based on Surrogate Safety Measures," Proceedings of the 96th Annual Meeting of the Transportation Research Board, Washington, D.C., January 2017.

Kim, S.-J., W. Kim and L. Rilett (2005). Calibration of Microsimulation Models Using Nonparametric Statistical Techniques. Transportation Research Record: Journal of the Transportation Research Board(1935): 111-119.

Knoop, V., S. Hoogendoorn and K. Adams (2009). Capacity Reductions at Incidents Sites On Motorways. EJTIR, 9 (4), 2009. 
Knoop, V. L., H. J. Van Zuylen and S. P. Hoogendoorn (2009). Microscopic traffic behaviour near incidents, Springer.

Koonce, P., L. Rodegerdts, K. Lee, S. Quayle, S. Beaird, C. Braud, J. Bonneson, P. Tarnoff and T. Urbanik (2008). Traffic signal timing manual.

Kwon, J., T. Barkley, R. Hranac, K. Petty and N. Compin (2011). Decomposition of Travel Time Reliability into Various Sources: Incidents, Weather, Work Zones, Special Events, and Base Capacity. Transportation Research Record: Journal of the Transportation Research Board(2229): 28-33.

Lardoux, J., R. Martinez, C. White, N. Gross, N. Patel and R. Meyer (2014). Adaptive Traffic Signal Control for Tarrytown Road in White Plains, New York.

Lin, P.-S. (2002). Signal Timing and Incident Management in Small Urban Areas. ITE 2002 Annual Meeting and Exhibit.

Lin, W., Y.-r. Su, F. Chang, K.-Y. Chen and S.-H. Huang (2012). Evaluation Approaches of Fuel Saving and Carbon Reduction Benefits for Arterial Signal Retiming. ITS Telecommunications (ITST), 2012 12th International Conference on, IEEE.

Lochmueller, B. (1995). Anchorage Signal System Upgrade-Final Report, Municipality of Anchorage, Alaska.

Lomax, T., D. Schrank, S. Turner and R. Margiotta (2003). Selecting Travel Reliability Measures. Texas Transportation Institute monograph (May 2003).

Lu, B., R. Xu, L. Hou and Z. Zhang (2011). Signal Control Optimization for Urban Traffic Against Incident-Induced Congestion. Control and Decision Conference (CCDC), 2011 Chinese, IEEE.

M.Rouphail, N., M. Hadi, B. Aghdashi, S. Khazraeian, J. L. Trask and Y. Xiao (2015). Dynamic Traffic Control Interventions for Enhanced Mobility and Economic Competitiveness Southesstern Transportation Research, Innovation, Development and Education Center.

Mahmassani, H. S., J. Kim, Y. Chen, Y. Stogios, A. Brijmohan and P. Vovsha (2014). Incorporating Reliability Performance Measures into Operations and Planning Modeling Tools, Transportation Research Board. 
Mahut, M., M. Florian, N. Tremblay, M. Campbell, D. Patman and Z. McDaniel (2004). Calibration and Application of a Simulation-Based Dynamic Traffic Assignment Model. Transportation Research Record: Journal of the Transportation Research Board(1876): 101-111.

Mai, E., G. List and R. Hranac (2012). Simulating the Travel Time Impact of Missed Transit Connections. Transportation Research Record: Journal of the Transportation Research Board(2274): 69-76.

Maki, P. (1999). Adverse Weather Traffic Signal Timing. Transportation Frontiers for the Next Millennium: 69th Annual Meeting of the Institute of Transportation Engineers.

Massahi, A., Hadi, M., Xiao, Y., and Chen, X., (January, 2016). Improved Model for Estimating Incident Impact on Urban Street Travel Time with Consideration of Upstream Intersection Capacity Reduction. Paper presented at the 95th Annual Meeting of the Transportation Research Board, National Research Council, Washington, D.C.

Massahi, A., Hadi, M., Adriana Cutillo, M., and Xiao, Y., (2017). Estimating the Capacity Impacts of Urban Street Incidents. Transportation Research Record (TRR) Journal, Journal of the Transportation Research Board.

May, A. D. (1990). Traffic Flow Fundamentals.

Maze, T., M. Agarwai and G. Burchett (2006). Whether Matters to Traffic Demand, Traffic Safety, and Traffic Operations and Flow. Transportation research record: Journal of the Transportation Research Board(1948): 170-176.

McCandless, J. (2007). The Calibration, Validation, and Comprision of VISSIM Simulations Using the Two -Fluid Model.

Michalopoulos, P. G. and K. Samartin (1998). Recent Developments of Advanced Technology in Freeway Management Projects. Traffic engineering \& control 39(3): 160165.

MNDOT (2004). FIRST Program Evaluation. Minnesota Department of Transportation. 
Murray, P., H. Mahmassani and K. Abdelghany (2001). Methodology for Assessing HighOccupancy Toll-Lane Usage and Network Performance. Transportation Research Record: Journal of the Transportation Research Board(1765): 8-15.

Nevers, B. L., K. M. Nguyen, S. M. Quayle, X. Zhou and J. Taylor (2013). The Effective Integration of Analysis, Modeling, and Simulation Tools.

Oregon, D. (2011). Protocol for Vissim Simulation, Oregon Department of Transportation.

Outlook, A. E. (2010). Energy information administration. Department of Energy.

Paramics, Q. (2015). Paramics. from http://www.paramics-online.com/.

Pesesky, L., D. Ismart, C. Huffman, Y.-C. Chiu, H. Zheng, E. Nava, V. Dixit, B. Wolshon, E. Radwan and W. Sampson (2012). Strategic Approaches at the Corridor and Network Level to Minimize Disruption From the Renewal Process.

PTV (2015). PTV Vissim 8 Highlights.

Rakha, H., M. Farzaneh, M. Arafeh and E. Sterzin (2008). Inclement Weather Impacts on Freeway Traffic Stream Behavior. Transportation Research Record: Journal of the Transportation Research Board(2071): 8-18.

Rämä, P. and J. Luoma (1997). Driver Acceptance Of Weather-Controlled Road Signs And Displays. Transportation Research Record: Journal of the Transportation Research Board(1573): 72-75.

Roelofs, T. and C. Brookes (2014). Synthesis of Intelligent Work Zone Practices.

Rojas, M., E. Sadeghvaziri, X. Jin (2016). A Comprehensive Review of Travel Behavior and Mobility Pattern Studies Using Mobile Phone Data. Transportation Research Record: Journal of the Transportation Research Board, No. 2563, Washington, D.C., 2016, pp. 7179.

Rouphail, N., B. Aghdashi, J. Trask, M. Hadi, Y. Xiao, S. Khazraeian, “ Dynamic Traffic Control Interventions for Enhanced Mobility and Economic Competitiveness-2013-009S", Final Report, Prepared for Southeastern Transportation Research, Innovation, 
Development, and Education Center (STRIDE) at the University of Florida, December 2015.

Sadeghvaziri, E., K. Haleem, P. Alluri, H. Fartash, A. Gan (2016). Effects of Different Guide Signs on Driver Behavior at Express Lane Entrance: A Driving Simulation Study. Poster presentation at The 95th annual meeting of the Transportation Research Board, National Research Council, Washington, D.C., January, 10-14.

Sadeghvaziri, E., M. Rojas, and X. Jin (2016). Exploring the Potential of Mobile Phone Data in Travel Pattern Analysis. Transportation Research Record: Journal of the Transportation Research Board, No. 2594, Washington, D.C., pp 27-34.

Sallman, D., K. Jeannotte, R. Margiotta and J. Strasser (2013). Operations Benefit/Cost Analysis TOPS-BC User's Manual: Providing Guidance to Practitioners in the Analysis of Benefits and Costs of Management and Operations Projects.

Seeherman, J. and A. Skabardonis (2013). Rethinking the Driver Population Factor: Examination of Freeways in California. Transportation Research Record: Journal of the Transportation Research Board(2395): 103-110.

Shams, K., Asgari, H. and X. Jin (2016). 'Valuation of Travel Time Reliability in Freight Transportation: A Review and Meta-Analysis of Stated preference Studies', Transportation Research Part A: Policy and Practice (102), pp. 228-243. https://doi.org/10.1016/j.tra.2016.08.001

Shams, K., Asgari, H., Hossan, M. and X. Jin (2017). "Value of Reliability for Road Freight Transportation: Evidence from a Stated Preference Survey in Florida". Transportation Research Record, Journal of Transportation Research Board, in press.

Sheehan, B., J. Colyer, J. Hunt and G. Jones (2012). ATDM Program Brief: Active Traffic Management. 13.

Sisiopiku, V. P., A. J. Sullivan, A. M. Abro, M. Shinouda, K. Mouskos and C. Barrett (2010). Development of a Dynamic Traffic Assignment Model to Evaluate Lane-Reversal Plans for I-65, University Transportation Center for Alabama. 
Skabardonis, A., H. Noeimi, K. Petty, D. Rydzewski, P. Varaiya and H. Al-Deek (1995). Freeway Service Patrol Evaluation. California Partners for Advanced Transit and Highways (PATH).

Sorensen, P., M. Wachs, E. Y. Min, A. Kofner and L. Ecola (2008). Moving Los Angeles: Short-Term Policy Options for Improving Transportation, Rand Corporation.

Sorrell, C. (2014). Planning and Evaluating Active Traffic Management Strategies. NCHRP Project 03-114.

Sprague, D. and J. Archambeau (2012). Adaptive Signal Timing: Comparison Between the Insync and QuicTrac Adaptive Signal Systems Installed in Colorado.

Sussman, J. M. (2000). What Have We Learned In ITS? Institute of Transportation Engineers International Meeting (2000: Irvine, Calif.). Transportation operations: moving into the 21 st century.

Systematics, C. (2013). Analytical Procedures For Determining The Impacts Of Reliability Mitigation Strategies, Transportation Research Board.

Tarry, S. and A. Graham (1995). The Role Of Evaluation in ATT development. IV: Evaluation of ATT systems. Traffic engineering \& control 36(12): 688-693.

Trafficware (2015). Trafficware Engineered by Naztec.

TSS (2015). AIMSUN, TSS-Transport Simulation Systems.

Turnbull, K. F. (2005). Managing Travel for Planned Special Events: First National Conference Proceedings.

Turnbull, K. F. (2005). Managing Travel for Planned Special Events: First National Conference Proceedings, December 1-3, 2004, New Orleans, Louisiana.

White, J. (2000). Traffic Signal Optimization for Tysons Corner Network Volume I. Evaluation and Summary, Virginia DOT.

Yang, S., F. Mei and M. A. Hadi (2008). Estimation of Incident Delays on Arterial Streets. Transportation Research Board 87th Annual Meeting. 
Young, S. E. and P. J. Tarnoff (2008). Guide to Benchmarking Operation Performance Measures, Summary of NCHRP 20-7. 15th World Congress on Intelligent Transport Systems and ITS America's 2008 Annual Meeting.

Zegeer, J., J. Bonneson, R. Dowling, P. Ryus, M. Vandehey, W. Kittelson, N. Rouphail, B. Schroeder, A. Hajbabaie and B. Aghdashi (2014). Incorporating Travel Time Reliability into the Highway Capacity Manual.

Zhang, H., S. Yang, F. Ni and B. Liu (2010). Freeway Traffic Incident Delay Analysis Based on Toll Data. 10th International Conference of Chinese Transportation Professionals, Beijing China.

Zhou, H. and Z. Tian (2012). Modeling Analysis of Incident and Roadway Clearance Time. Procedia-Social and Behavioral Sciences 43: 349-355.

Zhou, X. and J. Taylor (2014). DTALite: A queue-based mesoscopic traffic simulator for fast model evaluation and calibration. Cogent Engineering 1(1): 961345.

Balal, E., Long Cheu, R., Sarkodie-Gyan, T. (2016). A binary decision model for discretionary lane changing move based on fuzzy inference system. Transportation Research Part C: Emerging Technologies, Volume. 67, pp. 47-61.

Balal, E., Long Cheu, R., Sarkodie-Gyan, T., Miramontes, J. (2014). Analysis of discretionary lane changing parameters on freeways. International Journal of Transportation Science and Technology, Volume. 3, pp. 277-296.

Ghiasi, A., Ma, J., Zhou, F., Xiaopeng Li.,Speed (2017). Harmonization Algorithm Using Connected Autonomous Vehicles. Paper presented at the 96h annual meeting of the Transportation Research Board, National Research Council, Washington, D.C., January, 11-15. 
VITA

\section{AIDIN MASSAHI}

Tabriz, Iran

2004-2008

B.S., Civil Engineering

Imam Hossien University

Tehran, Iran

2008-2011

M.S., Roadway Design and Transportation Engineering KNTU University of Technology

Tehran, Iran

2013-2014

CSO representative, ITE Student Chapter at FIU

2013-2017

Doctoral Candidate and Graduate Assistant Transportation Engineering

Florida International University

Miami, Florida

2014-2015

Vice President, ITE Student Chapter at FIU

2014-2015

Social Coordinator, Iranian Student Chapter at FIU

2015-2016

President, ITE Student Chapter at FIU

2016

ITE International Best Student Chapter Award During Presidency

2016

Florida ITE Best Student Chapter Award During Presidency

2016-Present

Executive Board, Iranian American Foundation (IAF)

2017-Present

ITE International Student Committee

Traffic Operation Software:

VISSIM, AIMSUN, DTAlite, CORSIM, HCS, Synchro

Transportation Planning Software: $\quad$ VISSUM, Cube Voyager, Cube Avenue, ArcGIS Rodway Design Software: Civil 3D, Autodesk Land, SDR Map, AutoCAD

Econometrics \& Statistics:

Structural Software: SPSS, Statistica, R

Programing Software: ETABS, SAP

Visual Basic, MATLAB, C\# 
LANGUAGE:
Native: Azari, Persian; Fluent: English, Turkish; Familiar: Spanish, Arabic

\section{PUBLICATIONS AND PRESENTATIONS}

Massahi, A., Hadi, M., Adriana Cutillo, M., and Xiao, Y. (2017). Estimating the Capacity Impacts of Urban Street Incidents. Transportation Research Record (TRR) Journal, Journal of the Transportation Research Board.

Massahi, A., Ali, H., Koohifar, F., Baqersad, M., and Mohommadafzali, M. (2017). Implementing a Smartphone Application for Assessing the Raveling Performance of Asphalt Pavements. Advances in Materials Science and Engineering, (In Press).

Massahi, A., Hadji Hosseinlou, M., and Vakil Aliabadi, M., (2012). Developing Tehran Vehicles' Air Pollution Macroscopic Models and Presenting the Method for Estimating Pollutants' Emission Rates in Urban Networks. Science Series Data Report journal. Volume4, No4, part 7.

Mohammadafzali, M., Ali, H., Musselman, J., A. Massahi. (2017). Evaluation of Cracking Resistance and Durability of 100\% Reclaimed Asphalt Pavement Mixtures. Advances in Civil Engineering Journal.

Hadi, M., Xiao, Y., Tao, W., Massahi, A., Igbal,S, Jianmin, J.,Fartash, H., and Chen, X. (2015). Decision Support Systems for Transportation System Management and Operations (TSM\&O). Florida Department of Transportation (FDOT). Project BDV29977-09.

Hadi, M., Xiao, Y., Tao, W., Fakharian Qom, S., Massahi, A., Azizi, L., Jia J., and Iqbal M. S. (2016). Framework for Multi-Resolution Analyses of Advanced Traffic Management Strategies. Florida Department of Transportation (FDOT). Project BDV29977-19.

Massahi, A., Hadi, M., and Xiao, Y., (January, 2017). Estimating the Capacity Impacts of Urban Street Incidents. Paper presented at the $96^{\text {th }}$ Annual Meeting of the Transportation Research Board, National Research Council, Washington, D.C.

Massahi, A., Hadi, M., Xiao, Y., and Chen, X., (January, 2016). Improved Model for Estimating Incident Impact on Urban Street Travel Time with Consideration of Upstream Intersection Capacity Reduction. Paper presented at the $95^{\text {th }}$ Annual Meeting of the Transportation Research Board, National Research Council, Washington, D.C.

Massahi, A., Ali, H., and Koohifar, F., (January, 2016). Analysis of Pavement Raveling Using A Smart Phone. Paper presented at the $95^{\text {th }}$ Annual Meeting of the Transportation Research Board, National Research Council, Washington, D.C. 